\title{
Long-term manifestations and modifiers of prevalence estimates of the post-COVID-19 syndrome: A systematic review and meta-analysis.
}

Daniel De-la-Rosa-Martinez ${ }^{* 1,2}$, Marco Antonio Delaye-Martínez* ${ }^{2}$, Omar Yaxmehen BelloChavolla $^{3}$, Alejandro Sicilia-Andrade ${ }^{2}$, Isaac David Juárez-Cruz ${ }^{2}$, Carlos A. FermínMartínez $^{2}$, Alejandro Márquez-Salinas ${ }^{2}$, Enrique Cañedo-Guerra ${ }^{2}$, Luisa Fernández-Chirino ${ }^{4}$, Mónica Martínez-Gutiérrez², Daniel Eduardo Sandoval-Colin², Diana Vilar-Compte.

*De-la-Rosa-Martinez and Delaye-Martinez contributed equally to this manuscript.

1. Department of Infectious Diseases, Instituto Nacional de Cancerologia, Mexico City, México.

2. Plan de Estudios Combinados en Medicina (PECEM), Faculty of Medicine, Universidad Nacional Autonoma de Mexico, Mexico City, Mexico.

3. Division of Research, Instituto Nacional de Geriatría, Mexico City, Mexico

4. Faculty of Chemistry, Universidad Nacional Autónoma de México, Mexico City, Mexico

\section{Corresponding author:}

Diana Vilar-Compte, MD, MSc. Departamento de Infectologia, Instituto Nacional de Cancerologia. Av. San Fernando 22, Col. Sección XVI, Mexico City 14080, Mexico. T. +52 5556280400 ext. 12120. E-mail: diana_vilar@yahoo.com.mx 


\section{Abstract}

Background: Post-acute COVID-19 syndrome (PACS) is a multi-system disease comprising persistent symptomatology after the acute phase of infection. Long-term PACS effects significantly impact patient outcomes, but their incidence remains uncharacterized due to high heterogeneity between studies. Therefore, we aimed to summarize published data on PACS, characterizing the clinical presentation, prevalence, and modifiers of prevalence estimates.

Method: In this systematic review and meta-analysis, we research MEDLINE for original studies published from January 1st, 2020, to January 31st, 2021, that reported proportions of PACS manifestations. Studies were eligible for inclusion if they included patients aged $\geq 18$ years with confirmed COVID-19 by RT-PCR or antigen testing and a minimum follow-up of 21 days. The prevalence of individual manifestations across studies was pooled using random-effects meta-analysis. For evaluating determinants of heterogeneity, metaregression analysis was performed. This study was registered in PROSPERO (CRD42019125025).

Results: After screening 1,235 studies, we included 29 reports for analysis. Twenty-seven meta-analyses were performed, and 61 long-term manifestations were described. The pooled prevalence of PACS was $56 \%(95 \% \mathrm{Cl} 45-66 \%)$, with the most common manifestations being diminished health status, fatigue, asthenia, dyspnea, myalgias, hyposmia and dysgeusia. Most of the included studies presented high heterogeneity. After conducting the meta-regression analysis, we identified that age, gender, number of comorbidities, and reported symptoms significantly modify the prevalence estimation of PACS long-term manifestations.

Conclusion: PACS is inconsistently reported between studies, and population characteristics influence the prevalence estimates due to high heterogeneity. A systematized approach for the study of PACS is needed to characterize its impact adequately.

Funding: none 
medRxiv preprint doi: https://doi.org/10.1101/2021.10.17.21265123; this version posted October 18, 2021. The copyright holder for this preprint (which was not certified by peer review) is the author/funder, who has granted medRxiv a license to display the preprint in perpetuity.

It is made available under a CC-BY-NC 4.0 International license .

Keywords; COVID-19; PACS; SARS-CoV-2; Long COVID-19; Long-term; Prevalence

\section{INTRODUCTION}

Post-acute COVID-19 syndrome (PACS), also known as Long Covid, is a multi-system disease that occurs after the acute phase of COVID-19. Most patients with COVID-19 develop a mild acute illness lasting 15 to 20 days [1,2]; however, a variable proportion of patients persist with symptoms beyond four weeks from symptom onset. According to the World Health Organization, PACS "occurs in individuals with a history of probable or confirmed SARS CoV-2 infection, usually three months from the onset of COVID-19 with symptoms that last for at least two months and cannot be explained by an alternative diagnosis" [3]. Some studies have reported a prevalence of $10-33 \%$ of patients who undergo prolonged illness lasting from several weeks to months after acute COVID-19. Although these manifestations vary widely, the most common symptoms described are fatigue, headache, cough, breathlessness, joint pain, muscle and chest pain, weakness, gastrointestinal upset, rashes, chills, and olfactory and gustatory dysfunction. In addition, some neurocognitive impairments such as depression, anxiety, and post-traumatic stress syndrome have been highly reported.

Despite this growing available information, there is still uncertainty regarding the number and variety of the long-term effects attributable to PACS. Moreover, in agreement with previous observations in infections caused by Chikungunya, Ebola, Severe Acute Respiratory Syndrome Coronavirus (SARS-CoV-1), and Middle East respiratory syndrome (MERS) virus, these long-term manifestations could last for years after the acute infection [4-7]. Clinical characterization of PACS is still ongoing, and some risk factors have been preliminarily identified. Patients who developed severe COVID-19 are more likely to present long-term effects however, patients with an initial mild course are not free from developing new or persistent symptoms. In addition, some reports have placed women, patients admitted to the 
intensive care unit, and those with specific comorbidities as higher risk groups for developing long-term effects.

Recent data reporting the long-term effects of COVID-19 is showing a new landscape in SARS-CoV-2 infection. In addition, the proportion of patients with sequelae after COVID-19 who require specialized medical attention, rehabilitation programs, and absence from work is increasing, having profound medical, social, and economic implications which urgently need to be addressed by governments and other stakeholders. Here, we conducted a systematic review and metanalysis to evaluate data on the persistence of symptoms after acute COVID19, characterizing the clinical presentation and modifiers of prevalence estimates in PACS.

\section{METHODS}

\section{Search criteria}

A sensitive reference search was conducted using MEDLINE (via PubMed) to include manuscripts from January 1st, 2020, to January 31st, 2021. A preliminary search was made in PubMed, looking for relevant studies reporting the clinical status of patients recovered from COVID-19 several weeks after the acute illness. We focused on keywords included in each article to establish a general landscape of how "persistent symptoms" were described. Keywords included in our search were: post-discharge symptoms; long-COVID; recovery; post-COVID syndrome; sequels; long-term consequences; persistent symptoms; postCOVID; SARS-CoV-2 and COVID-19. Post-acute COVID-19 syndrome (PACS) was not included as a keyword, as it has recently been described. With the selected keywords, we designed a search algorithm in PubMed that combined Boolean operators (AND/OR), search filters for language (English and Spanish), and year of publication (2020-2021).

\section{Screening and data extraction}


Three authors (DDRM, MADM, IDJC) screened article titles and abstracts of the initial database search to identify those appropriate for complete text review. Pairs reviewed the full text of identified articles according to the following selection criteria:

1. Patients with confirmed COVID-19 by RT-PCR or rapid antigen test

2. Adult population (>18 years old)

3. Acute symptomatic illness during SARS-CoV-2 infection

4. A period of follow-up $\geq$ three weeks (21 days)

5. Reported the proportion of the specific persistent manifestations

Articles with confirmed reinfection cases were excluded from the analysis. For each included manuscript, data were extracted and summarized in a standardized database by reviewers.

\section{Quality assessment}

Joanna Briggs Institute critical appraisal tools were used to evaluate quality of the studies and PRISMA check list was used for reporting the findings. A third reviewer solved any disagreement if the initial reviewers reported discordant results. This review was prospectively registered in the PROSPERO database (Project number CRD42021243541).

\section{Variables}

We included demographic, clinical characteristics, and outcomes likely related to PACS in patients with previous COVID-19. Clinical signs and symptoms related to PACS were reported as proportions and described individually and classified according to the organ systems involved. Due to the nature of the pandemic and its rapid evolution, long-term manifestations associated with COVID-19 are not universally recognized or previously defined. Recently, the term PACS has been used as a reference in the literature. For this study and in agreement with the evidence of active viral infection and infectivity, PACS was defined as the persistence of symptoms $\geq 21$ days after SARS-CoV-2 infection diagnosis [8].

\section{Statistical analysis}


We calculated the pooled prevalence of symptoms for studies in which 1) the total number of patients with COVID-19 and the total number of those with persistent symptoms were reported by the authors and 2) at least two or more studies reported the specific long-term manifestation. We used a random-effects meta-analysis for calculating pooled prevalence estimates using the Hartung-Knapp-Sidik-Jonkman variance estimator, and the $1^{2}$ statistic test was calculated to quantify the heterogeneity of the studies [9]. $I^{2}$ values to establish the degree of heterogeneity were $>75 \%$ for high; $50-75 \%$ for substantial; $25-50 \%$ for moderate and $<25 \%$ for low heterogeneity. Forest plots were made to visualize the pooled prevalence of each long-term manifestation.

In order to diminish heterogeneity and improve meta-analytic pooling, we performed an outlier removing analysis as follows: We identified a study as an outlier if 1) its confidence interval did not overlap with the confidence interval of the pooled effect, 2) the upper bound of the $95 \% \mathrm{Cl}$ was lower than the lower bound of the pooled effect $\mathrm{Cl}$ or 3 ) the lower bound of the $95 \% \mathrm{Cl}$ was higher than the upper bound of the pooled effect $\mathrm{Cl}$ or 4 )A covariance ratio (CovRatio) value $<1$ of each manuscript [10]. CovRatio was calculated by dividing the variance of the pooled effect without each manuscript by the variance of the initial pooled effect. As a secondary objective, we looked for modifiers of PACS prevalence estimates: patients' age, gender, comorbidities, and the number of symptoms reported in each article. For this purpose, we performed a meta-regression analysis, and coefficients and 95\% confidence intervals were calculated for those symptoms reported at least by three authors [11]. Statistical analyses were performed using the meta package in $\mathrm{R}$ software version 4.1.1.

\section{RESULTS}

\section{Demographics characteristics}

Twenty-nine articles were included in the analysis after the systematic review and selection criteria of all screened studies [12-40]. Twenty-two (75.8\%), $3(10.3 \%), 3(10.3 \%)$ and 1 
(3.4\%) manuscripts were from Europe, America, Asia, and Africa, respectively. All articles were published during the second semester of 2020. The screening and selection process is shown in Figure 1. The number of patients included in the individual studies ranged from 23 to 4694 , with a female proportion between 27 to $75 \%$. The age range of participants was 27 to 70 years, and the period of follow-up since the onset of acute COVID-19 symptoms was 21 to 162 days. We did not identify studies with overlapping samples. The authors reported a wide range of comorbidities. Diabetes, hypertension, and obesity were reported in 17 (59\%), $17(59 \%)$, and $10(37 \%)$ articles, respectively. Concerning the number of comorbidities, 6 (21\%) manuscripts reported four or fewer, and $14(48 \%)$ reported more than five comorbidities. No information on comorbidities was available for 8 (28\%) manuscripts. Demographic and clinical characteristic of included studies are shown in Table 1

\section{Pooled prevalence of long-term PACS manifestations}

For the pooled prevalence of persistent symptoms, 27 meta-analyses were performed, and 61 long-term manifestations as part of the PACS spectrum were described (Table 2). The general PACS prevalence was 56\% $(95 \% \mathrm{Cl} 45-66 \%)$, which included any new or persistent symptom after three weeks from COVID-19 onset. Fatigue (49\%) and asthenia (32\%) were the most prevalent constitutional symptoms after acute COVID-19. For cognitive manifestations, attention disorders and memory loss were reported in $27 \%$ and $23 \%$ of patients, respectively, while anxiety and post-traumatic stress disorders were reported in around $30 \%$. The long-term prevalence and system involvement of PACS manifestations are shown in Figure 2.

The most frequent long-term respiratory symptoms were dyspnea (35\%), and sputum (20\%), followed by cough (19\%). Myocardial edema and diastolic dysfunction were present in 1 of 2 patients; nevertheless, this prevalence was reported only by one article. Gastrointestinal symptoms and rectal or urinary incontinence were reported in no more than $10 \%$ of patients. Ophthalmological sequelae occurred in $<20 \%$ of cases. Around 1 in 4 patients had myalgias 
and arthralgias as part of musculoskeletal manifestations. For cutaneous signs, loss of hair was the most frequent finding. Sixty percent of the included participants reported a diminished overall health perception, and sleep disorders were observed in one-third. In addition, the report by Kamal et al. included new-onset diabetes mellitus and renal failure as entities related to COVID-19 long-term spectrum [24]. Forest plots for specific symptoms are found in Supplementary material (figures). Five (19\%), 3 (11\%), and 19 (70\%) of metaanalyses reported low, substantial, and high heterogeneity, respectively. After outlier analysis, 11 (40\%) pooled analysis shown low, 1 (4\%) moderate, 6 substantial (22\%), and 9 (33\%) high heterogeneity (Table 3).

\section{Meta-regression for modifiers of PACS prevalence}

When evaluating modifying factors of PACS prevalence estimation, we found that age significantly raised prevalence estimation for fever, cough, and diarrhea within the evaluated manuscripts. Similarly, the proportion of female reported in the studies increased prevalence estimation of body aches, fever, and anosmia or hyposmia. Also, the prevalence estimation of arthralgias and red eye decreased with an increasing number of comorbidities. We also found that the general prevalence of PACS and cough increased according to the number of symptoms investigated by authors in each study, while the prevalence of asthenia and redeye decreased. Coefficients with $95 \%$ confidence intervals of the metaregression are shown in Supplementary Material (Table 1).

\section{DISCUSSION}

Prevalence and variety of long-term clinical manifestations of COVID-19 are not fully characterized. This analysis described the prevalence of 61 new or persistent manifestations after 21 days from the onset of COVID-19 symptoms and reported modifiers of prevalence estimation, which may influence PACS spectrum. To date, there is not an accepted period for establishing long-term complications. Some institutions such as the Centers for Disease Control and Prevention (CDC) in the USA propose four weeks to define the long-term effects 
of COVID-19, while the guidelines of the National Institute for Health and Care Excellence (NICE) in England considers a more extended period of $>12$ weeks to classify the long-term manifestations. Notably, sequelae of COVID-19 persist for longer than six months and are higher than other respiratory infections, including influenza [41]. Definition of clinical stages during COVID-19 has proven to be challenging. However, Van Kampen, JJA et al, observed that shedding of SARS-CoV-2 virus has an average duration of 15 days, with a probability of $<5 \%$ isolation of viable virus after three weeks from symptom onset. Based on this observation, a period of 21 days may reflect the conclusion of acute COVID-19 [42].

We found that 1 in 2 patients with COVID-19 will develop new or persistent clinical manifestations compatible with PACS. In a previous meta-analysis, Lopez-Leon S. et al. reported PACS prevalence as high as $80 \%$ [43]. In contrast, Taquet et al. found in a cohort of 273,618 COVID-19 survivors that $57 \%$ of patients had one or more long-COVID clinical manifestations during a 6-month period, although this number decreased to $36 \%$ after three months, this prevalence continues to be a large burden of long-term sequels [41]. Similar to our findings, Groff et al, reported a median PASC frequency at 1 month of $54.0 \%$ (45.0\%$69.0 \%$;); at $2-5$ months, $55.0 \%(34.8 \%-65.5 \%)$; and at 6 or more months, $54.0 \%(31.0 \%$ $67.0 \%)[44]$

Even though more reports are published on PACS, the heterogeneity of studies continues to limit the quality of evidence. In $19(70 \%)$ of the pooled prevalence meta-analysis performed, heterogeneity was high; this large heterogeneity was expected first due to subjectivity involving specific clinical manifestations and second to the inability of standardized tools to evaluate some long-term manifestations. To deal with this high heterogeneity, an analysis to determine outlier manuscripts was performed, showing a $50 \%$ decrease of all high-level pooled prevalence analyses. This high heterogeneity could be explained to prevalence modifiers identified in the meta-regression analysis, including age, gender, comorbidities, and the number of evaluated symptoms [45]. By controlling sources of heterogeneity that 
influence prevalence estimates for PACS and its manifestations, future studies are more likely to provide unbiased prevalence estimates of PACS, which may better inform the follow-up of patients after acute COVID-19.

Little is known about the clinical characteristics of patients who develop PACS. A report by the CDC indicates that nearly one of five out-setting patients with no comorbidities and age from 18-34 years did not return to usual health within three weeks of testing due to the persistence of symptoms [46]. Interestingly, Dennis et al. observed that $70 \%$ of the individuals at low risk of COVID-19 related mortality developed impairment in one or more organs in the following four months after the onset of symptoms; most of those patients were young women with a low prevalence of comorbidities and ambulatory treatment for COVID19 [47]. These characteristics are opposite of what is described in acute COVID-19, in which comorbid men have a higher susceptibility to develop severe COVID-19 [48]. Notably, we found that the percentage of women reported in the manuscripts analyzed increased the prevalence of body aches, fever, anosmia, or hyposmia, while reported age, modified prevalence in an inverse relation for fever, cough, and diarrhea. Thus, intrinsic patient characteristics and the design of previous studies may contribute to gender and age differences.

Immunity-modulated gender disparities may contribute to the differences found in the prognosis of COVID-19 and PACS onset. The infection response process follows a series of steps that initiate with the entrance of the SARS-CoV-2 virus to cells by attaching to ACE-2 receptor and its posterior recognition by Toll-like receptors (TLR) 3, 7, 8, and 9, RIG-I and MDA5, with a subsequent triggering of type I interferon (I-IFN) response and NLRP3 inflammasome activation, these signals culminate in pyroptosis and inflammation response [49]. Some reports establish an internal capacity of some coronaviruses to diminish and delay I-IFN response, leading to increased viral replication and severe illness [50]. Likewise, it has been proposed that the imbalance of this normal response may culminate in other 
disease manifestations such as multisystem inflammatory syndrome and post-acute COVID syndrome. In addition, higher titles of anti-IFN antibodies are found in men compared to women [51]. Thus, a more robust type I-IFN response in women and young individuals may partially explain the better immunological response during acute COVID-19 and posterior more adverse long-term effects in this population [52].

Precise estimates of PACS prevalence and its manifestations will be pivotal to planning public health policies aimed at mitigating the long-term socio-economic impact of Long COVID on public and private healthcare systems around the world [53]. Given the wide spectrum of PACS on different body systems, a multidisciplinary approach will be required to facilitate the recovery and rehabilitation of patients after COVID-19 [54]. Until now, there is no specific treatment or management to treat PACS manifestations; however, recent data on the impact of COVID-19 vaccinations on symptom recovery in PACS is promising and may provide a viable pathway to reduce its burden despite the emergence of new SARS-CoV-2 variants or breakthrough infections [55-57]

Our study has several limitations. Available data were primarily extracted from observational studies with no standardized symptoms in MEDLINE. Also, post-acute COVID-19 syndrome definitions were not available, contributing to increased risks of bias. Results should be interpreted with caution as high expected heterogeneity was found in most meta-analytic results. Despite that, a strength of our study was the meta-regression analysis conducted to explore for modifiers of prevalence estimates for post-acute COVID-19 syndrome. In addition, our findings are similar to those previously reported. Comorbidities were not available for eight manuscripts for meta-regression analysis, and one article did not specify the proportion of women in the population.

Our findings may help standardize populations to study PACS after acute COVID-19 in future studies and to provide better estimates of long-term manifestations, influencing PACS- 
medRxiv preprint doi: https://doi.org/10.1101/2021.10.17.21265123; this version posted October 18, 2021. The copyright holder for this preprint

(which was not certified by peer review) is the author/funder, who has granted medRxiv a license to display the preprint in perpetuity. It is made available under a CC-BY-NC 4.0 International license.

related public policy. Longitudinal studies on incident COVID-19 cases require standardized approaches regarding the definition of PACS and long-term manifestations, consistent follow-up times across studies and populations with diverse clinical spectrums of COVID-19 to better understand the incidence of PACS, its burden and the impact on patients and healthcare systems. Data available on request from the authors.

Funding support: No specific founding was disclosed

Conflict of interest's disclosers: No conflict of interests was declared by the authors 


\section{REFERENCES}

1. Cohen PA, Hall LE, John JN, Rapoport AB. The Early Natural History of SARS-CoV-2 Infection. Mayo Clinic Proceedings 2020; 95: 1124-6.

2. Wu Z, McGoogan JM. Characteristics of and Important Lessons From the Coronavirus Disease 2019 (COVID-19) Outbreak in China: Summary of a Report of 72314 Cases From the Chinese Center for Disease Control and Prevention. JAMA 2020; 323: 1239.

3. WHO. A clinical case definition of post COVID-19 condition by a Delphi consensus, 6 October 2021.

4. Moldofsky H, Patcai J. Chronic widespread musculoskeletal pain, fatigue, depression and disordered sleep in chronic post-SARS syndrome; a case-controlled study. BMC Neurol 2011; 11: 37. 5. Lam MH-B. Mental Morbidities and Chronic Fatigue in Severe Acute Respiratory Syndrome Survivors: Long-term Follow-up. Arch Intern Med 2009; 169: 2142.

6. Guillot X, Ribera A, Gasque P. Chikungunya-Induced Arthritis in Reunion Island: A Long-Term Observational Follow-Up Study Showing Frequently Persistent Joint Symptoms, Some Cases of Persistent Chikungunya Immunoglobulin M Positivity, and No Anticyclic Citrullinated Peptide Seroconversion After 13 Years. J Infect Dis 2020; 222: 1740-4.

7. Clark DV, Kibuuka H, Millard M, et al. Long-term sequelae after Ebola virus disease in Bundibugyo, Uganda: a retrospective cohort study. Lancet Infect Dis 2015; 15: 905-12.

8. Nalbandian A, Sehgal K, Gupta A, et al. Post-acute COVID-19 syndrome. Nat Med 2021; 27: 60115.

9. Langan D, Higgins JPT, Jackson D, et al. A comparison of heterogeneity variance estimators in simulated random $\square$ effects meta $\square$ analyses. Res Syn Meth 2019; 10: 83-98.

10. Viechtbauer W, Cheung MW-L. Outlier and influence diagnostics for meta-analysis. Res Synth Method 2010; 1: 112-25.

11. Viechtbauer W. Conducting Meta-Analyses in R with the metafor Package. J Stat Soft 2010; 36: $1-48$.

12. Barón-Sánchez J, Santiago C, Goizueta-San Martín G, Arca R, Fernández R. Afectación del sentido del olfato y el gusto en la enfermedad leve por coronavirus (COVID-19) en pacientes españoles. Neurología 2020; 35: 633-8.

13. Blair PW, Brown DM, Jang M, et al. The Clinical Course of COVID-19 in the Outpatient Setting: A Prospective Cohort Study. Open Forum Infectious Diseases 2021; 8: ofab007.

14. Boscolo-Rizzo P, Borsetto D, Fabbris C, et al. Evolution of Altered Sense of Smell or Taste in Patients With Mildly Symptomatic COVID-19. JAMA Otolaryngol Head Neck Surg 2020; 146: 729. 15. Carfi A, Bernabei R, Landi F, for the Gemelli Against COVID-19 Post-Acute Care Study Group. Persistent Symptoms in Patients After Acute COVID-19. JAMA 2020; 324: 603.

16. Caronna E, Ballvé A, Llauradó A, et al. Headache: A striking prodromal and persistent symptom, predictive of COVID-19 clinical evolution. Cephalalgia 2020; 40: 1410-21. 
17. Carvalho-Schneider C, Laurent E, Lemaignen A, et al. Follow-up of adults with noncritical COVID-19 two months after symptom onset. Clinical Microbiology and Infection 2021; 27: 258-63. 18. Gambini G, Savastano MC, Savastano A, et al. Ocular Surface Impairment After Coronavirus Disease 2019: A Cohort Study. Cornea 2021; 40: 477-83.

19. Garrigues E, Janvier P, Kherabi Y, et al. Post-discharge persistent symptoms and health-related quality of life after hospitalization for COVID-19. Journal of Infection 2020; 81: e4-6.

20. Gerkin RC, Ohla K, Veldhuizen MG, et al. Recent Smell Loss Is the Best Predictor of COVID-19 Among Individuals With Recent Respiratory Symptoms. Chemical Senses 2021; 46: bjaa081. 21. Halpin SJ, McIvor C, Whyatt G, et al. Postdischarge symptoms and rehabilitation needs in survivors of COVID $\square 19$ infection: A cross $\square$ sectional evaluation. J Med Virol 2021; 93: 1013-22. 22. Huang L, Zhao P, Tang D, et al. Cardiac Involvement in Patients Recovered From COVID-2019 Identified Using Magnetic Resonance Imaging. JACC: Cardiovascular Imaging 2020; 13: 2330-9. 23. Jacobs LG, Gourna Paleoudis E, Lesky-Di Bari D, et al. Persistence of symptoms and quality of life at 35 days after hospitalization for COVID-19 infection. Madeddu G, ed. PLoS ONE 2020; 15: e0243882.

24. Kamal M, Abo Omirah M, Hussein A, Saeed H. Assessment and characterisation of post-COVID19 manifestations. Int J Clin Pract 2021; 75: e13746.

25. Konstantinidis I, Delides A, Tsakiropoulou E, Maragoudakis P, Sapounas S, Tsiodras S. ShortTerm Follow-Up of Self-Isolated COVID-19 Patients with Smell and Taste Dysfunction in Greece: Two Phenotypes of Recovery. ORL J Otorhinolaryngol Relat Spec 2020; 82: 295-303.

26. Landi F, Carfì A, Benvenuto F, et al. Predictive Factors for a New Positive Nasopharyngeal Swab Among Patients Recovered From COVID-19. American Journal of Preventive Medicine 2021; 60: 13-9.

27. Myall KJ, Mukherjee B, Castanheira AM, et al. Persistent Post-COVID-19 Interstitial Lung Disease. An Observational Study of Corticosteroid Treatment. Annals ATS 2021; 18: 799-806. 28. Niklassen AS, Draf J, Huart C, et al. COVID $\square$ 19: Recovery from Chemosensory Dysfunction. A Multicentre study on Smell and Taste. The Laryngoscope 2021; 131: 1095-100.

29. Park S, Lee C-W, Park D-I, et al. Detection of SARS-CoV-2 in Fecal Samples From Patients With Asymptomatic and Mild COVID-19 in Korea. Clinical Gastroenterology and Hepatology 2021; 19: 1387-1394.e2.

30. Pellaud C, Grandmaison G, Pham Huu Thien HP, et al. Characteristics, comorbidities, 30-day outcome and in-hospital mortality of patients hospitalised with COVID-19 in a Swiss area - a retrospective cohort study. Swiss Med Wkly 2020; 150: w20314.

31. Raman B, Cassar MP, Tunnicliffe EM, et al. Medium-term effects of SARS-CoV-2 infection on multiple vital organs, exercise capacity, cognition, quality of life and mental health, post-hospital discharge. EClinicalMedicine 2021; 31: 100683.

32. Sonnweber T, Sahanic S, Pizzini A, et al. Cardiopulmonary recovery after COVID-19: an observational prospective multicentre trial. Eur Respir J 2021; 57: 2003481.

33. Taboada M, Moreno E, Cariñena A, et al. Quality of life, functional status, and persistent symptoms after intensive care of COVID-19 patients. British Journal of Anaesthesia 2021; 126: e110-3. 34. Tomasoni D, Bai F, Castoldi R, et al. Anxiety and depression symptoms after virological clearance of COVID $\square$ 19: A cross $\square$ sectional study in Milan, Italy. J Med Virol 2021; 93: 1175-9. 35. Townsend L, Dowds J, O'Brien K, et al. Persistent Poor Health after COVID-19 Is Not Associated with Respiratory Complications or Initial Disease Severity. Annals ATS 2021; 18: $997-$ 1003.

36. Townsend L, Dyer AH, Jones K, et al. Persistent fatigue following SARS-CoV-2 infection is common and independent of severity of initial infection. Madeddu G, ed. PLOS ONE 2020; 15: 
e0240784.

37. Vaira LA, Hopkins C, Petrocelli M, et al. Smell and taste recovery in coronavirus disease 2019 patients: a 60-day objective and prospective study. J Laryngol Otol 2020; 134: 703-9.

38. Villarreal IM, Morato M, Martínez-RuizCoello M, et al. Olfactory and taste disorders in healthcare workers with COVID-19 infection. Eur Arch Otorhinolaryngol 2021; 278: 2123-7.

39. Weerahandi H, Hochman KA, Simon E, et al. Post-Discharge Health Status and Symptoms in Patients with Severe COVID-19. J GEN INTERN MED 2021; 36: 738-45.

40. Zhao Y, Shang Y, Song W, et al. Follow-up study of the pulmonary function and related physiological characteristics of COVID-19 survivors three months after recovery. EClinicalMedicine 2020; 25: 100463.

41. Taquet M, Dercon Q, Luciano S, Geddes JR, Husain M, Harrison PJ. Incidence, co-occurrence, and evolution of long-COVID features: A 6-month retrospective cohort study of 273,618 survivors of COVID-19. Kretzschmar MEE, ed. PLoS Med 2021; 18: e1003773.

42. van Kampen JJA, van de Vijver DAMC, Fraaij PLA, et al. Duration and key determinants of infectious virus shedding in hospitalized patients with coronavirus disease-2019 (COVID-19). Nat Commun 2021; 12: 267.

43. Lopez-Leon S, Wegman-Ostrosky T, Perelman C, et al. More than 50 long-term effects of COVID-19: a systematic review and meta-analysis. Sci Rep 2021; 11: 16144.

44. Groff D, Sun A, Ssentongo AE, et al. Short-term and Long-term Rates of Postacute Sequelae of SARS-CoV-2 Infection: A Systematic Review. JAMA Netw Open 2021; 4: e2128568.

45. Sudre CH, Murray B, Varsavsky T, et al. Attributes and predictors of long COVID. Nat Med 2021; 27: 626-31.

46. Tenforde MW, Kim SS, Lindsell CJ, et al. Symptom Duration and Risk Factors for Delayed Return to Usual Health Among Outpatients with COVID-19 in a Multistate Health Care Systems Network — United States, March-June 2020. MMWR Morb Mortal Wkly Rep 2020; 69: 993-8. 47. Dennis A, Wamil M, Alberts J, et al. Multiorgan impairment in low-risk individuals with postCOVID-19 syndrome: a prospective, community-based study. BMJ Open 2021; 11: e048391.

48. Brandi ML, Giustina A. Sexual Dimorphism of Coronavirus 19 Morbidity and Lethality. Trends in Endocrinology \& Metabolism 2020; 31: 918-27.

49. Sette A, Crotty S. Adaptive immunity to SARS-CoV-2 and COVID-19. Cell 2021; 184: 861-80. 50. Schultze JL, Aschenbrenner AC. COVID-19 and the human innate immune system. Cell 2021; 184: 1671-92.

51. Bastard P, Rosen LB, Zhang Q, et al. Autoantibodies against type I IFNs in patients with lifethreatening COVID-19. Science 2020; 370: eabd4585.

52. Brodin P. Immune determinants of COVID-19 disease presentation and severity. Nat Med 2021; 27: 28-33.

53. Menges D, Ballouz T, Anagnostopoulos A, et al. Burden of post-COVID-19 syndrome and implications for healthcare service planning: A population-based cohort study. Simuunza MC, ed. PLOS ONE 2021; 16: 0254523.

54. Wade DT. Rehabilitation after COVID-19: an evidence-based approach. Clin Med 2020; 20: 35965.

55. Tran V-T, Perrodeau E, Saldanha J, Pane I, Ravaud P. Efficacy of COVID-19 Vaccination on the Symptoms of Patients With Long COVID: A Target Trial Emulation Using Data From the ComPaRe e-Cohort in France. SSRN Journal 2021.

56. Arnold DT, Milne A, Samms E, Stadon L, Maskell NA, Hamilton FW. Symptoms After COVID19 Vaccination in Patients With Persistent Symptoms After Acute Infection: A Case Series. Ann Intern Med 2021; 174: 1334-6.

57. Antonelli M, Penfold RS, Merino J, et al. Risk factors and disease profile of post-vaccination 
medRxiv preprint doi: https://doi.org/10.1101/2021.10.17.21265123; this version posted October 18, 2021. The copyright holder for this preprint (which was not certified by peer review) is the author/funder, who has granted medRxiv a license to display the preprint in perpetuity.

It is made available under a CC-BY-NC 4.0 International license .

SARS-CoV-2 infection in UK users of the COVID Symptom Study app: a prospective, communitybased, nested, case-control study. The Lancet Infectious Diseases 2021: S1473309921004606.

Figure 1. PRISMA flow diagram for search, screening, and manuscript selection.

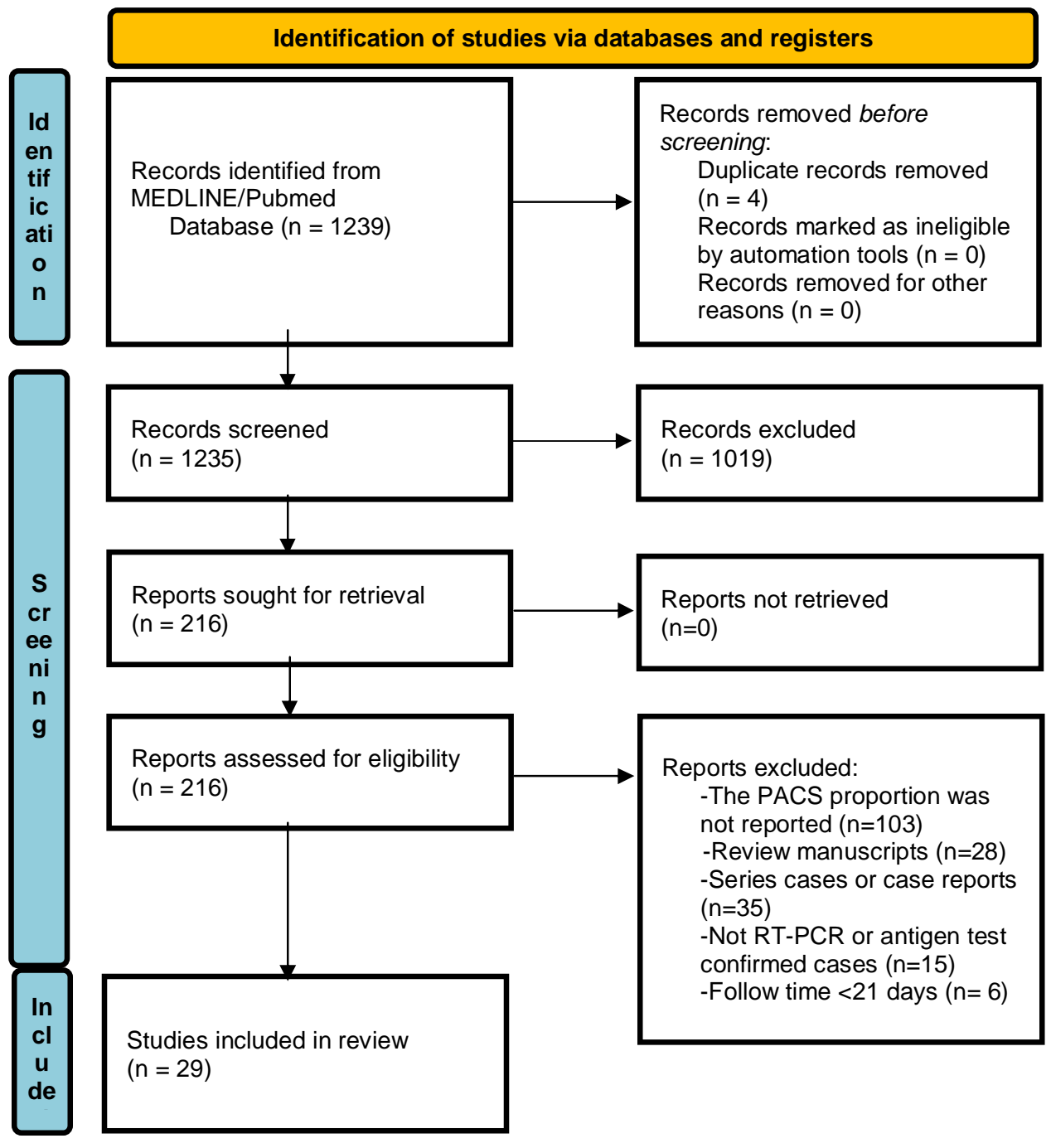


medRxiv preprint doi: https://doi.org/10.1101/2021.10.17.21265123; this version posted October 18, 2021. The copyright holder for this preprint (which was not certified by peer review) is the author/funder, who has granted medRxiv a license to display the preprint in perpetuity. It is made available under a CC-BY-NC 4.0 International license.

Figure 2. Pooled prevalence estimates of long-term manifestations after acute COVID-

19 
medRxiv preprint doi: https://doi.org/10.1101/2021.10.17.21265123; this version posted October 18, 2021. The copyright holder for this preprint (which was not certified by peer review) is the author/funder, who has granted medRxiv a license to display the preprint in perpetuity.

It is made available under a CC-BY-NC 4.0 International license .

\section{LONG-TERM MANIFESTATIONS AFTER ACUTE COVID-19}

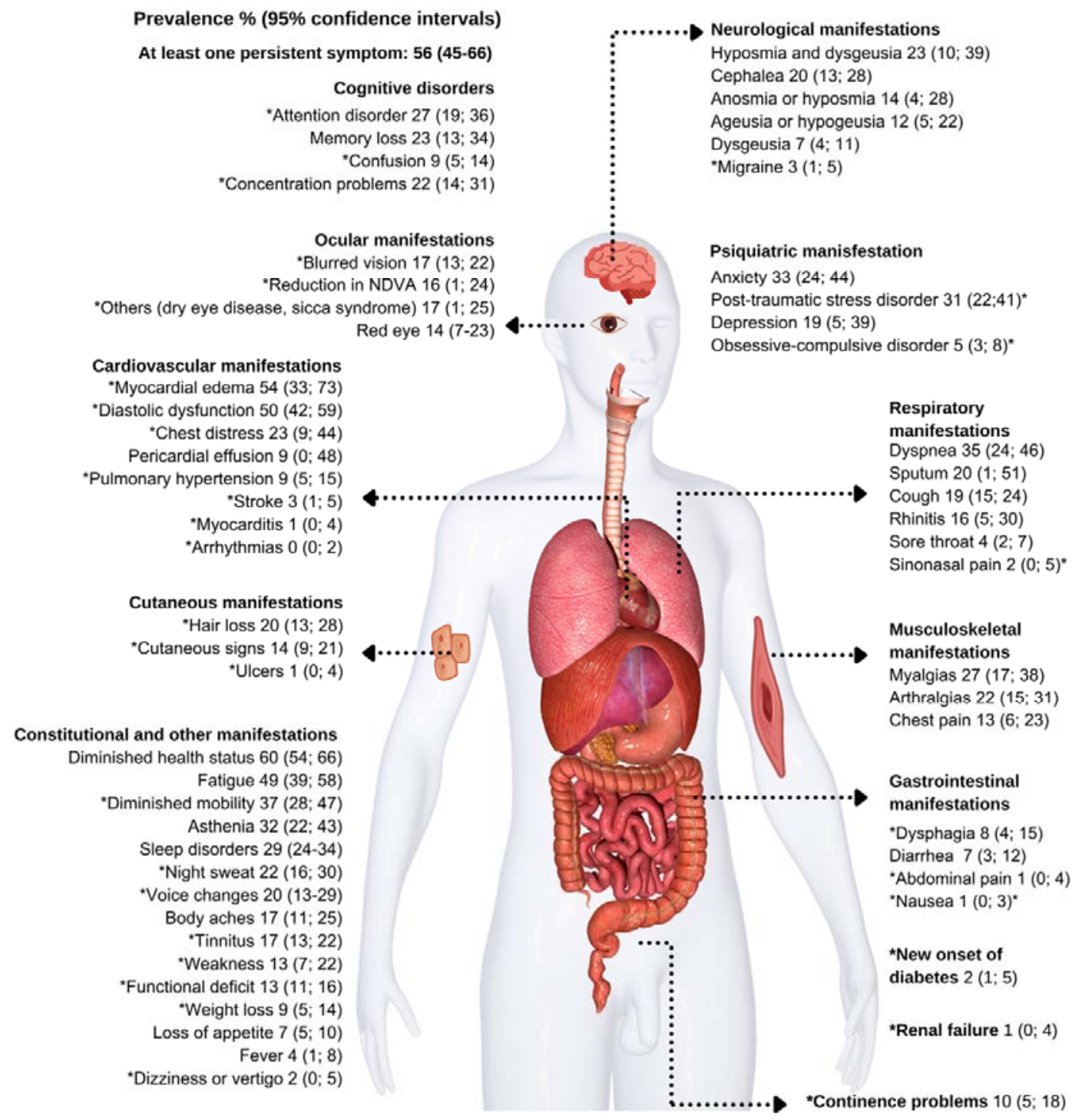

${ }^{*}$ Only reported in one manuscript. ${ }^{* *}$ Continence involves both urinary and fecal continence. NDVA;

Near Distance Visual Acuity 
Table 1. General and demographic characteristics of included studies.

\begin{tabular}{|c|c|c|c|c|c|c|c|c|c|c|}
\hline Author & Country & Design & $\begin{array}{l}\text { Sample } \\
\text { size (N) }\end{array}$ & $\begin{array}{c}\text { Gender } \\
(\% \\
\text { female })\end{array}$ & $\begin{array}{l}\text { Mean age } \\
\text { years } \\
\text { (SD or } \\
\text { IQR) }\end{array}$ & Population & Comorbidities & PACS symptoms & $\begin{array}{l}\text { Clinical } \\
\text { spectrum } \\
\text { (systems } \\
\text { involved) }\end{array}$ & $\begin{array}{l}\text { Follow } \\
\text {-up } \\
\text { (days) }\end{array}$ \\
\hline $\begin{array}{l}\text { Konstantinidis, et } \\
\text { al. }\end{array}$ & Greece & $\begin{array}{c}\text { Cohort } \\
\text { (prospective) }\end{array}$ & 79 & 51 & $30.7(5.3)$ & Ambulatory & Not specified & $\begin{array}{c}\text { Nasal obstruction, } \\
\text { olfactory and gustatory } \\
\text { dysfunctions, rhinitis and, } \\
\text { rhinorrhea. }\end{array}$ & $\begin{array}{l}\text { Neurological } \\
\text { Respiratory }\end{array}$ & 30 \\
\hline $\begin{array}{l}\text { Carvalho- } \\
\text { Schneider, et al. }\end{array}$ & France & $\begin{array}{c}\text { Cohort } \\
\text { (prospective) }\end{array}$ & 150 & 56 & $49(15)$ & $\begin{array}{l}\text { Ambulatory } \\
\text { and } \\
\text { hospitalized }\end{array}$ & $\begin{array}{c}0 \text { comorbidities }(46 \%), 1 \\
\text { comorbidity }(35 \%), 2 \\
\text { comorbidities }(19 \%)\end{array}$ & $\begin{array}{l}\text { Olfactory and gustatory } \\
\text { dysfunctions, chest pain, } \\
\text { diarrhea, weight loss, } \\
\text { cutaneous signs, dyspnea, } \\
\text { arthralgia, palpitations, } \\
\text { fever, myalgia, cephalea, } \\
\text { asthenia and vomiting. }\end{array}$ & $\begin{array}{l}\text { Neurological } \\
\text { Respiratory } \\
\text { Musculoskeletal } \\
\text { Gastrointestinal } \\
\text { Cardiovascular } \\
\text { Constitutional } \\
\text { Cutaneous }\end{array}$ & 30,60 \\
\hline Kamal, et al. & Egypt & $\begin{array}{l}\text { Cross- } \\
\text { sectional }\end{array}$ & 287 & 64 & $32.3(8.5)$ & $\begin{array}{l}\text { Ambulatory } \\
\text { and } \\
\text { hospitalized }\end{array}$ & $\begin{array}{c}\text { No comorbidities }(70.7 \%), \\
\text { hypertension }(7.7 \%) \text {, diabetes } \\
(5.2 \%) \text {, obesity }(35.5 \%), \\
\text { dyslipidemia }(1.4 \%), \\
\text { rheumatoid arthritis }(1 \%), \\
\text { hypothyroidism }(1 \%) \text {, asthma } \\
(1 \%) \text {, peptic ulcer }(0.7 \%), \\
\text { arrhythmia }(0.7 \%), \text { smoking } \\
(9.8 \%)\end{array}$ & $\begin{array}{c}\text { Fatigue, anxiety, } \\
\text { arthralgia, cephalea, chest } \\
\text { pain, depression, dyspnea, } \\
\text { new onset diabetes, } \\
\text { blurred vision, tinnitus, } \\
\text { fever, obsessive- } \\
\text { compulsive disorder, } \\
\text { migraine, stroke, renal } \\
\text { failure, myocarditis, } \\
\text { arrhythmia. }\end{array}$ & $\begin{array}{l}\text { Constitutional } \\
\text { Psychiatric } \\
\text { Musculoskeletal } \\
\text { Neurological } \\
\text { Respiratory } \\
\text { Endocrinological } \\
\text { Cardiovascular } \\
\text { Renal }\end{array}$ & 21 \\
\hline Landi, et al. & Italy & $\begin{array}{c}\text { Cohort } \\
\text { (prospective) }\end{array}$ & 131 & 39 & $\begin{array}{c}55.8 \\
(14.8)\end{array}$ & Hospitalized & $\begin{array}{l}\text { Hypertension (29\%), COPD } \\
(9.1 \%) \text {, heart failure }(6.1 \%) \text {, } \\
\text { diabetes (5.3\%), renal failure } \\
(3.1 \%), \text { smoking }(9.8 \%)\end{array}$ & $\begin{array}{l}\text { Fatigue, dyspnea, } \\
\text { arthralgia, cough, red eye, } \\
\text { rhinitis, cephalea, loss of } \\
\text { appetite, sore throat, } \\
\text { diarrhoea, and olfactory } \\
\text { and gustatory } \\
\text { dysfunctions. }\end{array}$ & $\begin{array}{l}\text { Constitutional } \\
\text { Respiratory } \\
\text { Ocular } \\
\text { Musculoskeletal } \\
\text { Neurological } \\
\text { Gastrointestinal }\end{array}$ & 55 \\
\hline Garrigues, et al. & France & $\begin{array}{l}\text { Cross- } \\
\text { sectional }\end{array}$ & 120 & 38 & $\begin{array}{c}63.2 \\
(15.7)\end{array}$ & Hospitalized & $\begin{array}{c}\text { Hypertension: }(46.7 \%), \\
\text { overweight or obesity }(47.5 \%) \text {, } \\
\text { diabetes }(21.7 \%)\end{array}$ & $\begin{array}{l}\text { Fatigue, dyspnea, memory } \\
\text { loss, sleep disorders, } \\
\text { attention disorder, hair } \\
\text { loss, cough, chest pain, }\end{array}$ & $\begin{array}{l}\text { Constitutional } \\
\text { Respiratory } \\
\text { Neurological } \\
\text { Musculoskeletal }\end{array}$ & 110 \\
\hline
\end{tabular}




\begin{tabular}{|c|c|c|c|c|c|c|c|c|c|c|}
\hline & & & & & & & & $\begin{array}{l}\text { and olfactory and } \\
\text { gustatory dysfunctions. }\end{array}$ & & \\
\hline Tomasini, et al. & Italy & $\begin{array}{l}\text { Cross- } \\
\text { sectional }\end{array}$ & 105 & 27 & $\begin{array}{c}55 \\
(43-65)\end{array}$ & Hospitalized & Not specified & $\begin{array}{c}\text { Gastrointestinal } \\
\text { symptoms, asthenia, } \\
\text { anxiety, memory disorders, } \\
\text { depression, burning pain, } \\
\text { dyspnea, and olfactory and } \\
\text { gustatory dysfunctions, } \\
\text { chest pain, cephalea, } \\
\text { constipation, tinnitus, } \\
\text { insomnia, } \\
\text { palpitations, NSTEMI, } \\
\text { cough, and sore throat. }\end{array}$ & $\begin{array}{l}\text { Constitutional } \\
\text { Psychiatric } \\
\text { Neurological } \\
\text { Respiratory } \\
\text { Gastrointestinal } \\
\text { Musculoskeletal } \\
\text { Cardiovascular }\end{array}$ & $30-90$ \\
\hline Carfi, et al. & Italy & $\begin{array}{l}\text { Cross- } \\
\text { sectional }\end{array}$ & 143 & 37 & $\begin{array}{c}56.5 \\
(16.6)\end{array}$ & Hospitalized & $\begin{array}{c}\text { Hypertension (35\%), thyroid } \\
\text { disease }(18.2 \%) \text {, immune } \\
\text { disorders }(11.2 \%), \text { COPD } \\
(9.1 \%) \text {, diabetes (7\%), } \\
\text { cardiovascular disease }(4.9 \%) \text {, } \\
\text { cancer }(3.5 \%) \text {, atrial fibrillation } \\
\text { (2.8\%), heart failure }(2.8 \%), \\
\text { renal failure }(2.1 \%), \text { stroke } \\
\text { (1.4\%), active smoking } \\
(10.5 \%)\end{array}$ & $\begin{array}{c}\text { Fatigue, dyspnea, } \\
\text { arthralgias, chest pain, } \\
\text { cough, anosmia, sicca } \\
\text { syndrome, rhinitis, red } \\
\text { eyes, dysgeusia, } \\
\text { headache, sputum, lack of } \\
\text { appetite, sore throat, } \\
\text { vertigo, myalgia and } \\
\text { diarrhea. }\end{array}$ & $\begin{array}{l}\text { Constitutional } \\
\text { Respiratory } \\
\text { Musculoskeletal } \\
\text { Neurological } \\
\text { Gastrointestinal }\end{array}$ & 60 \\
\hline Vaira, et al. & Italy & $\begin{array}{c}\text { Cohort } \\
\text { (prospective) }\end{array}$ & 138 & 51 & $51.2(8.8)$ & $\begin{array}{l}\text { Ambulatory } \\
\text { and } \\
\text { hospitalized }\end{array}$ & $\begin{array}{c}\text { Cardiovascular disease } \\
(26.8 \%), \text { pulmonary disease } \\
(15.2 \%) \text {, diabetes }(10.9 \%), \\
\text { obesity }(29 \%)\end{array}$ & $\begin{array}{c}\text { Olfactory and gustatory } \\
\text { dysfunction }\end{array}$ & Neurological & 60 \\
\hline Halpin, et al. & UK & $\begin{array}{l}\text { Cross- } \\
\text { sectional }\end{array}$ & 100 & 46 & $\begin{array}{c}\text { Ward } \\
\text { patients: } \\
70.5 \\
(20-93) \\
\\
\text { ICU: } \\
58.5 \\
(34-84)\end{array}$ & Hospitalized & $\begin{array}{c}\text { Hypertension }(41 \%) \text {, obesity } \\
\text { (24\%), diabetes }(29 \%), \\
\text { gastrointestinal disease }(25 \%), \\
\text { cancer ( } 28 \%) \text {, heart failure } \\
(5 \%), \text { mental health condition } \\
(19 \%), \text { osteoarthritis }(13 \%), \\
\text { rheumatologic disease }(14 \%), \\
\text { CKD (15\%), } \\
\text { immunosuppressive condition } \\
(15 \%), \text { asthma (13\%), } \\
\text { neurologic disease (12\%), } \\
\text { COPD (8\%), obstructive sleep } \\
\text { apnea (7\%), hyperlipidemia } \\
(4 \%), \text { ischemic heart disease } \\
\text { (10\%), tachyarrhythmias }\end{array}$ & $\begin{array}{l}\text { Fatigue, dyspnea, post- } \\
\text { traumatic stress syndrome, } \\
\text { concentration and memory } \\
\text { alterations, } \\
\text { anxiety/depression, pain or } \\
\text { discomfort, cough, } \\
\text { continence problems, } \\
\text { dysphagia, voice changes, } \\
\text { appetite problems, and } \\
\text { diminished mobility. }\end{array}$ & $\begin{array}{c}\text { Constitutional } \\
\text { Respiratory } \\
\text { Psychiatric } \\
\text { Neurological } \\
\text { Urological } \\
\text { Gastrointestinal }\end{array}$ & 48 \\
\hline
\end{tabular}




\begin{tabular}{|c|c|c|c|c|c|c|c|c|c|c|}
\hline & & & & & & & $\begin{array}{c}\text { (12\%), valvular heart disease } \\
(3 \%), \text { venous } \\
\text { thromboembolism }(5 \%), \\
\text { thyroid disease }(5 \%), \\
\text { gynecological disease }(3 \%), \\
\text { hematological disease }(10 \%), \\
\text { infectious disease }(6 \%)\end{array}$ & & & \\
\hline $\begin{array}{c}\text { Barón-Sánchez, et } \\
\text { al. }\end{array}$ & Spain & $\begin{array}{l}\text { Cross- } \\
\text { sectional }\end{array}$ & 31 & 61 & $\begin{array}{c}44.6 \\
(13.08)\end{array}$ & Ambulatory & Not specified. & Hyposmia. & Neurological & 38.47 \\
\hline Villarreal, et al. & Spain & $\begin{array}{l}\text { Cohort } \\
\text { (retrospectiv } \\
\text { e) }\end{array}$ & 230 & 85 & $\begin{array}{c}43 \\
(18-62)\end{array}$ & $\begin{array}{l}\text { Ambulatory } \\
\text { and } \\
\text { hospitalized }\end{array}$ & Not specified. & Olfactory dysfunctions & Neurological & 30 \\
\hline Zhao, et al. & China & $\begin{array}{c}\text { Cohort } \\
\text { (retrospectiv } \\
\text { e) }\end{array}$ & 55 & 42 & $\begin{array}{c}47.7 \\
(15.49)\end{array}$ & Hospitalized & $\begin{array}{c}\text { Hypertension }(10.91 \%), \\
\text { diabetes }(3.64 \%), \\
\text { cardiovascular disease } \\
(3.64 \%), \text { smoking }(3.64 \%), \\
\text { others }(3.64 \%) .\end{array}$ & $\begin{array}{c}\text { Gastrointestinal } \\
\text { symptoms, cephalea, } \\
\text { fatigue, dyspnea, gustatory } \\
\text { dysfunctions, cough, and } \\
\text { sputum. }\end{array}$ & $\begin{array}{l}\text { Gastrointestinal } \\
\text { Neurological } \\
\text { Constitutional } \\
\text { Respiratory }\end{array}$ & $64-93$ \\
\hline Pellaud, et al. & $\begin{array}{l}\text { Switzerla } \\
\text { nd }\end{array}$ & $\begin{array}{l}\text { Cohort } \\
\text { (retrospectiv } \\
\text { e) }\end{array}$ & 196 & $N / E$ & $\begin{array}{c}70 \\
(60-80)\end{array}$ & Hospitalized & $\begin{array}{c}\text { Hypertension }(60 \%) \text {, diabetes } \\
(27 \%) \text {, obesity }(21 \%) \text {, coronary } \\
\text { heart disease }(13 \%) \text {, sleep } \\
\text { apnea syndrome }(13 \%), \\
\text { cardiac insufficiency }(11 \%), \\
\text { COPD }(8 \%) \text {, cancer }(8 \%), \\
\text { immunosuppression }(5 \%), \\
\text { smoking }(29 \%) .\end{array}$ & $\begin{array}{l}\text { Asthenia, respiratory } \\
\text { symptoms, olfactory and } \\
\text { gustatory dysfunction. }\end{array}$ & $\begin{array}{l}\text { Constitutional } \\
\text { Respiratory } \\
\text { Neurological }\end{array}$ & 30 \\
\hline $\begin{array}{l}\text { Boscolo-Rizzo, et } \\
\text { al. }\end{array}$ & Italy & $\begin{array}{l}\text { Cross- } \\
\text { sectional }\end{array}$ & 187 & 55 & $\begin{array}{c}56 \\
(20-89)\end{array}$ & $\begin{array}{l}\text { Ambulatory } \\
\text { and } \\
\text { hospitalized }\end{array}$ & Not specified. & $\begin{array}{l}\text { Gustatory and olfactory } \\
\text { dysfunction, cough, } \\
\text { dyspnea, cephalea, } \\
\text { congested nose, } \\
\text { myalgias/arthralgias, loss } \\
\text { of appetite, felt tired, sore } \\
\text { throat, dizziness, diarrhea, } \\
\text { sinonasal pain, abdominal } \\
\text { pain, chest pain, fever, } \\
\text { nausea. }\end{array}$ & $\begin{array}{l}\text { Neurological } \\
\text { Respiratory } \\
\text { Musculoskeletal } \\
\text { Constitutional } \\
\text { Gastrointestinal }\end{array}$ & 30 \\
\hline Park, et al. & Korea & $\begin{array}{c}\text { Cohort } \\
\text { (prospective) }\end{array}$ & 46 & 46 & $\begin{array}{c}26 \\
(18-57)\end{array}$ & Hospitalized & $\begin{array}{c}\text { Reflux (13\%), gastritis }(6.5 \%) \text {, } \\
\text { irritable bowel disease }(6.5 \%) \text {, } \\
\text { fatty liver }(2.1 \%) \text {, others } \\
(6.5 \%) .\end{array}$ & $\begin{array}{l}\text { Sputum, cough, } \\
\text { rhinorrhea, cephalea, } \\
\text { fatigue, chest pain, } \\
\text { myalgia, nasal obstruction, } \\
\text { anosmia, loss of appetite, }\end{array}$ & $\begin{array}{l}\text { Respiratory } \\
\text { Neurological } \\
\text { Constitutional } \\
\text { Musculoskeletal }\end{array}$ & 52 \\
\hline
\end{tabular}




\begin{tabular}{|c|c|c|c|c|c|c|c|c|c|c|}
\hline & & & & & & & & fever. & & \\
\hline Huang, et al. & China & Case-control & 26 & 62 & $\begin{array}{c}38 \\
(32-45)\end{array}$ & Hospitalized & Hypertension (8\%) & $\begin{array}{l}\text { Precordial chest pain, } \\
\text { palpitations, chest distress. }\end{array}$ & Cardiovascular & 47 \\
\hline Caronna, et al. & Spain & $\begin{array}{c}\text { Cohort } \\
\text { (prospective) }\end{array}$ & 130 & 51 & $\begin{array}{c}53.9 \\
(16.4)\end{array}$ & $\begin{array}{l}\text { Ambulatory } \\
\text { and } \\
\text { hospitalized }\end{array}$ & Migraine (70\%) & Cephalea. & Neurological & 42 \\
\hline Townsend, et al. & Ireland & $\begin{array}{l}\text { Cross- } \\
\text { sectional }\end{array}$ & 128 & 54 & $\begin{array}{l}49.5 \\
(15)\end{array}$ & $\begin{array}{l}\text { Ambulatory/ } \\
\text { hospitalized }\end{array}$ & Anxiety/depression (7.8\%) & $\begin{array}{l}\text { Fatigue, not returning to } \\
\text { full health. }\end{array}$ & Constitutional & 42 \\
\hline Gambini, et al. & Italy & $\begin{array}{c}\text { Cohort } \\
\text { (prospective) }\end{array}$ & 114 & 33 & $\begin{array}{c}56.2 \\
(14.2)\end{array}$ & Hospitalized & $\begin{array}{c}\text { Hypertension (43.8\%), } \\
\text { diabetes ( } 26.6 \%) \text {, autoimmune } \\
\text { disease }(10.9 \%) .\end{array}$ & $\begin{array}{l}\text { Reduction of near distance } \\
\text { visual acuity, red eye }\end{array}$ & Ocular & 60.3 \\
\hline Sonnweber, et al. & Austria & $\begin{array}{c}\text { Cohort } \\
\text { (prospective) }\end{array}$ & 145 & 45 & $\begin{array}{c}57 \\
(14)\end{array}$ & $\begin{array}{l}\text { Ambulatory } \\
\quad \text { and } \\
\text { hospitalized }\end{array}$ & $\begin{array}{c}\text { Hypertension }(30 \%), \\
\text { hypercholesterolemia }(19 \%) \text {, } \\
\text { diabetes }(17 \%) \text {, cancer }(12 \%) \text {, } \\
\text { asthma }(7 \%) \text {, CKD }(7 \%), \\
\text { immunodeficiency }(6 \%), \\
\text { COPD }(6 \%), \text { chronic liver } \\
\text { disease }(6 \%) \text {, interstitial lung } \\
\text { disease }(1 \%) . .\end{array}$ & $\begin{array}{l}\text { Dyspnea, sleep disorders, } \\
\text { night sweet, pain, olfactory } \\
\text { dysfunctions, cough, } \\
\text { diarrhea/vomiting. }\end{array}$ & $\begin{array}{c}\text { Respiratory } \\
\text { Constitutional } \\
\text { Neurological } \\
\text { Gastrointestinal }\end{array}$ & $60-100$ \\
\hline Jacobs, et al. & USA & $\begin{array}{c}\text { Cohort } \\
\text { (prospective) }\end{array}$ & 183 & 39 & $\begin{array}{c}57 \\
(48-68)\end{array}$ & Hospitalized & $\begin{array}{c}\text { Hypertension (47\%), obesity } \\
(49.2 \%), \text { overweight }(36.1 \%) \text {, } \\
\text { diabetes }(28.4 \%) \text {, cardiac } \\
\text { disease }(27.9 \%) \text {, dyslipidemia } \\
(10.9 \%), \text { asthma }(10.4 \%), \\
\text { cancer }(9.8 \%), \\
\text { immunodeficiency }(4.4 \%), \\
\text { hypothyroidism }(4.4 \%), \\
\text { psychiatric disorder }(4.4 \%) \text {, } \\
\text { COPD (3.8\%), obstructive } \\
\text { sleep apnea (3.3\%), } \\
\text { thromboembolic disease } \\
(1.6 \%) .\end{array}$ & $\begin{array}{l}\text { Fatigue, dyspnea, cough, } \\
\text { myalgias, cephalea, } \\
\text { phlegm, ageusia, olfactory } \\
\text { dysfunction, confusion, } \\
\text { eye irritation, diarrhea, } \\
\text { ulcers, fever. }\end{array}$ & $\begin{array}{l}\text { Constitutional } \\
\text { Respiratory } \\
\text { Musculoskeletal } \\
\text { Neurological } \\
\text { Ocular } \\
\text { Gastrointestinal } \\
\text { Cutaneous }\end{array}$ & 35 \\
\hline Taboada, et al. & Spain & $\begin{array}{c}\text { Cohort } \\
\text { (prospective) }\end{array}$ & 91 & 35 & $\begin{array}{c}64.5 \\
(10.4)\end{array}$ & Hospitalized & $\begin{array}{c}\text { Hypertension (55.5\%), } \\
\text { dislipidemia (44\%), obesity } \\
\text { (38.5\%), diabetes (23.1\%), } \\
\text { cardiomyopathy }(20.9 \%) \\
\text { COPD }(8.8 \%) \text {, asthma }(6.6 \%) .\end{array}$ & $\begin{array}{l}\text { Dyspnea, myalgias, } \\
\text { asthenia, insomnia, } \\
\text { arthralgias, } \\
\text { cough, olfactory } \\
\text { dysfunctions, chest pain }\end{array}$ & $\begin{array}{l}\text { Respiratory } \\
\text { Musculoskeletal } \\
\text { Constitutional } \\
\text { Neurological }\end{array}$ & 180 \\
\hline Gerkin, et al. & USA & Cross- & 4694 & $75^{*}$ & 40.6 & Not specified & Diabetes $(2 \%)$, heart disease & Olfactory dysfunctions, & Neurological & 40 \\
\hline
\end{tabular}




\begin{tabular}{|c|c|c|c|c|c|c|c|c|c|c|}
\hline & & sectional & & & $(12)^{*}$ & & $\begin{array}{c}\text { (0.3\%), hypertension }(8 \%), \\
\text { lung disease }(0.5 \%), \\
\text { neurological disease }(11 \%), \\
\text { obesity }(1 \%) \text {, seasonal } \\
\text { allergies }(17 \%) \text {, smoking } \\
(63 \%) \text {, chronic sinus problems } \\
(3 \%) \text {, cancer }(0.5 \%)\end{array}$ & $\begin{array}{l}\text { runny nose, sore throat, } \\
\text { not recovered from } \\
\text { respiratory symptom }\end{array}$ & Respiratory & \\
\hline Blair, et al. & USA & $\begin{array}{c}\text { Cohort } \\
\text { (prospective) }\end{array}$ & 118 & 57 & $\begin{array}{c}56 \\
(50-63)\end{array}$ & $\begin{array}{l}\text { Ambulatory } \\
\text { and } \\
\text { hospitalized }\end{array}$ & $\begin{array}{l}\text { Hypertension (39\%), smoking } \\
(26 \%) \text {, diabetes }(16.1 \%), \\
\text { asthma or COPD (15.3\%), } \\
\text { solid tumor (15.3\%), HIV } \\
\text { (6.6\%), hematologic } \\
\text { malignancy (5.1\%), CKD } \\
(3.4 \%) \text {, history of myocardial } \\
\text { infarction (1.7\%). }\end{array}$ & Fatigue, dry cough & $\begin{array}{l}\text { Constitutional } \\
\text { Respiratory }\end{array}$ & $28-60$ \\
\hline Niklassen, et al. & $\begin{array}{l}\text { German } \\
y, \text { Italy }\end{array}$ & $\begin{array}{c}\text { Cohort } \\
\text { (prospective) }\end{array}$ & 111 & 46 & $\begin{array}{l}44.5 \\
(15)\end{array}$ & Not specified & Not specified & $\begin{array}{l}\text { Olfactory and gustatory } \\
\text { dysfunctions. }\end{array}$ & Neurological & $\begin{array}{l}28,45 \\
77-162\end{array}$ \\
\hline Raman, et al. & UK & Case-control & 88 & 40 & $\begin{array}{c}55.4 \\
(13.2)^{\star}\end{array}$ & Hospitalized & $\begin{array}{c}\text { Hypertension (37.9\%), asthma } \\
(34.5 \%) \text {, diabetes mellitus } \\
(15.5 \%) \text { depression }(5.2 \%), \\
\text { COPD }(5.2 \%), \text { coronary artery } \\
\text { disease }(3.4 \%), \\
\text { cerebrovascular disease } \\
(1.7 \%), \text { previous cancer } \\
(3.4 \%) .\end{array}$ & Dyspnea, fatigue. & $\begin{array}{l}\text { Respiratory } \\
\text { Constitutional }\end{array}$ & $60-90$ \\
\hline Townsend, et al. & Ireland & $\begin{array}{l}\text { Cross- } \\
\text { sectional }\end{array}$ & 153 & 57 & $\begin{array}{c}48 \\
(35-59)\end{array}$ & $\begin{array}{l}\text { Ambulatory } \\
\text { and } \\
\text { Hospitalized }\end{array}$ & Not specified & $\begin{array}{l}\text { Fatigue, not returning to } \\
\text { full health. }\end{array}$ & Constitutional & 75 \\
\hline Myall, et al. & UK & $\begin{array}{c}\text { Cohort } \\
\text { (prospective) }\end{array}$ & 35 & 71 & $60.5(10.7)$ & Hospitalized & $\begin{array}{c}\text { Hypertension (31.4\%), Obesity } \\
(25.7 \%) \text {, diabetes }(22.9 \%), \\
\text { asthma }(22.9 \%) \text {, CKD }(5.8 \%), \\
\text { COPD }(5.8 \%), \text { HIV }(2.9 \%) .\end{array}$ & Functional deficit. & Respiratory & 42 \\
\hline Weerahandi, et al. & USA & $\begin{array}{c}\text { Cohort } \\
\text { (prospective) }\end{array}$ & 161 & 37 & $\begin{array}{c}62 \\
(50-67)\end{array}$ & Hospitalized & $\begin{array}{c}\text { Smoker }(30.4 \%), \text { CKD }(8.1 \%) \text {, } \\
\text { cancer }(7.5 \%) \text {, arterial } \\
\text { coronary disease }(9.3 \%), \\
\text { diabetes }(36.7 \%) \text {, cardiac } \\
\text { failure }(5 \%), \text { hyperlipidemia } \\
(46.6 \%), \text { hypertension } \\
(60.3 \%) \text {, asthma or COPD } \\
(24.2 \%) \text {, overweight }(30.4 \%),\end{array}$ & $\begin{array}{l}\text { Dyspnea, diminished } \\
\text { general health status, } \\
\text { diminished physical health, } \\
\text { diminished mental health. }\end{array}$ & $\begin{array}{l}\text { Respiratory } \\
\text { Psychiatric }\end{array}$ & 37 \\
\hline
\end{tabular}




\begin{tabular}{|l|l|l|l|l|l|l|l|l|l|}
\hline & & & & & & & obesity (41\%). & & \\
\hline
\end{tabular}

${ }^{*}$ From COVID-19 group.

Red eyes included: burning eye, foreign body sensation, watering eye, dry eye disease and eye irritation.

Olfactory dysfunctions include anosmia, hyposmia, dysosmia, phantosmia.

Gustatory dysfunctions include: ageusia, hypogeusia, dysgeusia

Olfactory and gustatory dysfunction include both olfactory and gustatory manifestations if they were reported together in the same article.

Chronic obstructive pulmonary disease (COPD), chronic kidney disease (CKD), Human Immunodeficiency Virus (HIV).

$(\mathrm{N} / \mathrm{E})$ Not specified 
Table 2. Pooled prevalence estimates of new or persistent manifestation after acute COVID-19

\begin{tabular}{|c|c|c|c|c|c|c|c|c|c|c|c|}
\hline Symptom & Studies & Cases & $\begin{array}{l}\text { Sample } \\
\text { Size }\end{array}$ & $\begin{array}{l}\text { Prevalence \% } \\
\quad(95 \mathrm{Cl} \%)\end{array}$ & $12(\%)$ & Symptom & Studies & Cases & $\begin{array}{l}\text { Sample } \\
\text { Size }\end{array}$ & $\begin{array}{l}\text { Prevalence \% } \\
\quad(95 \mathrm{Cl} \%)\end{array}$ & $\begin{array}{l}12 \\
(\%)\end{array}$ \\
\hline $\begin{array}{l}\text { PACS (at least one persistent } \\
\text { symptom) }\end{array}$ & 15 & 2923 & 5586 & $56(45-66)$ & 98 & \multicolumn{6}{|c|}{ Gastrointestinal manifestations } \\
\hline \multicolumn{6}{|l|}{ Constitutional manifestations } & Dysphagia & 1 & 8 & 100 & $8(4 ; 15)$ & - \\
\hline Fatigue & 12 & 828 & 1534 & $49(39 ; 58)$ & 93 & Diarrhea & 5 & 59 & 774 & $7(3 ; 12)$ & 82 \\
\hline Asthenia & 5 & 199 & 649 & $32(22 ; 43)$ & 89 & Abdominal pain & 1 & 2 & 187 & $1(0 ; 4)$ & - \\
\hline Night sweat & 1 & 32 & 145 & $22(16 ; 30)$ & - & Nausea & 1 & 1 & 187 & $1(0 ; 3)$ & - \\
\hline Body aches & 3 & 62 & 350 & $17(11 ; 25)$ & 68 & \multicolumn{6}{|c|}{ Musculoskeletal manifestations } \\
\hline Tinnitus & 1 & 48 & 287 & $17(13 ; 22)$ & - & Myalgias & 4 & 133 & 470 & $27(17 ; 38)$ & 84 \\
\hline Weakness & 1 & 11 & 84 & $13(7 ; 22)$ & - & Arthralgias & 6 & 225 & 963 & $22(15 ; 31)$ & 88 \\
\hline Weight loss & 1 & 13 & 150 & $9(5 ; 14)$ & - & Chest pain & 8 & 174 & 1050 & $13(6 ; 23)$ & 93 \\
\hline Loss of appetite & 4 & 34 & 442 & $7(5 ; 10)$ & 2 & \multicolumn{6}{|c|}{ Endocrinological manifestations } \\
\hline Fever & 5 & 45 & 853 & $4(1 ; 8)$ & 86 & New onset diabetes & 1 & 7 & 287 & $2(1 ; 5)$ & - \\
\hline Dizziness or vertigo & 1 & 3 & 187 & $2(0 ; 5)$ & - & \multicolumn{6}{|l|}{ Renal manifestations } \\
\hline \multicolumn{6}{|l|}{ Neurological manifestations } & Renal failure & 1 & 4 & 287 & $1(0 ; 4)$ & - \\
\hline Hyposmia and dysgeusia & 4 & 99 & 426 & $23(10 ; 39)$ & 92 & \multicolumn{6}{|l|}{ Others } \\
\hline Cephalea & 8 & 240 & 1117 & $20(13 ; 28)$ & 89 & Continence problems & 1 & 10 & 100 & $10(5 ; 18)$ & - \\
\hline Anosmia or hyposmia & 12 & 1747 & 4358 & $14(4 ; 28)$ & 99 & Sleep disorders & 3 & 103 & 356 & $29(24 ; 34)$ & 0 \\
\hline Ageusia or hypogeusia & 4 & 77 & 591 & $12(5 ; 22)$ & 91 & Voice changes & 1 & 20 & 100 & $20(13 ; 29)$ & - \\
\hline Dysgeusia & 3 & 19 & 269 & $7(4 ; 11)$ & 18 & \multicolumn{6}{|l|}{ General health perception } \\
\hline Migraine & 1 & 8 & 287 & $3(1 ; 5)$ & - & $\begin{array}{l}\text { Diminished general health } \\
\text { status. }\end{array}$ & 2 & 169 & 281 & $60(54 ; 66)$ & 0 \\
\hline \multicolumn{6}{|l|}{ Cognitive disorders } & Diminished mobility & 1 & 37 & 100 & $37(28 ; 47)$ & - \\
\hline Attention disorder & 1 & 32 & 120 & $27(19 ; 36)$ & - & Functional deficit & 1 & 110 & 837 & $13(11 ; 16)$ & - \\
\hline Memory loss & 3 & 77 & 325 & $23(13 ; 34)$ & 82 & \multicolumn{6}{|l|}{ Respiratory manifestations } \\
\hline Confusion & 1 & 16 & 183 & $9(5 ; 14)$ & - & Dyspnea & 13 & 573 & 1733 & $35(24 ; 46)$ & 96 \\
\hline
\end{tabular}




\begin{tabular}{|c|c|c|c|c|c|c|c|c|c|c|c|}
\hline Concentration problems & 1 & 22 & 100 & $22(14 ; 31)$ & - & Sputum & 3 & 54 & 284 & $20(1 ; 51)$ & 96 \\
\hline \multicolumn{5}{|l|}{ Psychiatric manifestations } & & Cough & 8 & 192 & 965 & $19(15 ; 24)$ & 66 \\
\hline Anxiety & 2 & 138 & 392 & $33(24 ; 44)$ & 73 & Rhinitis** & 4 & 51 & 372 & $16(5 ; 30)$ & 89 \\
\hline Post-traumatic & 1 & 31 & 100 & $31(22 ; 41)$ & - & Sore throat & 2 & 13 & 296 & $4(2 ; 7)$ & 0 \\
\hline Depression & 2 & 93 & 392 & $19(5 ; 39)$ & 94 & Sinonasal pain & 1 & 3 & 187 & $2(0 ; 5)$ & - \\
\hline Obsessive-compulsive disorder & 1 & 14 & 287 & $5(3 ; 8)$ & - & \multicolumn{6}{|c|}{ Cardiovascular manifestations } \\
\hline \multicolumn{6}{|l|}{ Ocular manifestations } & Myocardial edema & 1 & 14 & 26 & $54(33 ; 73)$ & - \\
\hline Blurred vision & 1 & 49 & 287 & $17(13 ; 22)$ & - & Diastolic dysfunction & 1 & 73 & 145 & $50(42 ; 59)$ & - \\
\hline Reduction in NDVA & 1 & 18 & 114 & $16(1 ; 24)$ & - & Chest distress & 1 & 6 & 26 & $23(9 ; 44)$ & - \\
\hline $\begin{array}{l}\text { Others (dry eye disease, sicca } \\
\text { syndrome) }\end{array}$ & 1 & 19 & 114 & $17(1 ; 25)$ & - & Pericardial effusion & 2 & 8 & 171 & $9(0 ; 48)$ & 95 \\
\hline Red eye* & 3 & 55 & 406 & $14(7-23)$ & 80 & Pulmonary hypertension & 1 & 13 & 145 & $9(5 ; 15)$ & - \\
\hline \multicolumn{6}{|l|}{ Cutaneous manifestations } & Stroke & 1 & 8 & 287 & $3(1 ; 5)$ & - \\
\hline Hair loss & 1 & 24 & 120 & $20(13 ; 28)$ & - & Arrhythmias & 1 & 1 & 287 & $0(0 ; 2)$ & - \\
\hline Cutaneous signs & 1 & 21 & 150 & $14(9 ; 21)$ & - & Myocarditis & 1 & 4 & 287 & $1(0 ; 4)$ & - \\
\hline Ulcers & 1 & 2 & 183 & $1(0 ; 4)$ & - & & & & & & \\
\hline
\end{tabular}

${ }^{*}$ Red eye includes eye irritation, foreign body sensation, burning eye, watering eye

${ }^{*}$ Rhinitis includes congested nose and runny nose.

Near Distance Visual Acuity (NDVA); Non-ST segment elevation myocardial infarction (NSTEMI) 
Table 3. Outlier removing analysis of pooled prevalence estimates for PACS and individual long-term manifestations.

\begin{tabular}{|c|c|c|c|}
\hline Symptom & $\begin{array}{l}\text { Prevalence \% } \\
\text { (95 Cl\%) }\end{array}$ & $12(\%)$ & $\begin{array}{l}\text { Outlier studies } \\
\text { excluded }\end{array}$ \\
\hline PACS & $53(47-69)$ & 82 & 7 \\
\hline Fatigue & $48(44-53)$ & 41 & 4 \\
\hline Asthenia & $36(32-41)$ & 0 & 1 \\
\hline Body aches & $17(11 ; 25)$ & 68 & 0 \\
\hline Loss of appetite & $7(5-10)$ & 2 & 0 \\
\hline Fever & $2(1 ; 4)$ & 0 & 1 \\
\hline Hyposmia and dysgeusia & 29 (24-35) & 0 & 1 \\
\hline Cephalea & $20(13 ; 28)$ & 89 & 0 \\
\hline Anosmia or hyposmia & $13(9-17)$ & 78 & 2 \\
\hline Ageusia or hypogeusia & $8(4-13)$ & 59 & 1 \\
\hline Dysgeusia & $7(4-11)$ & 18 & 0 \\
\hline Memory loss & $18(13-23)$ & 0 & 1 \\
\hline Red eye* & $14(7-23)$ & 80 & 0 \\
\hline Diarrhea & $5(3-7)$ & 12 & 1 \\
\hline Myalgias & $31(21-42)$ & 83 & 1 \\
\hline Arthralgias & $26(20-32)$ & 75 & 1 \\
\hline Chest pain & $15(10-19)$ & 51 & 2 \\
\hline Sleep disorders & $29(24 ; 34)$ & 0 & 0 \\
\hline Dyspnea & $36(29-43)$ & 83 & 5 \\
\hline Sputum & $7(0-24)$ & 89 & 1 \\
\hline Cough & $18(14-22)$ & 55 & 1 \\
\hline Rhinitis** & $9(6-13)$ & 0 & 1 \\
\hline
\end{tabular}

${ }^{*}$ Red eye includes eye irritation, foreign body sensation, burning eye, watering eye

${ }^{* *}$ Rhinitis includes congested nose and runny nose. 
Supplementary table 1. Meta Regression for modifiers of prevalence estimates for PACS and individual long-term manifestations.

\begin{tabular}{|c|c|c|c|c|c|c|c|c|}
\hline \multirow[b]{2}{*}{ Symptom } & \multicolumn{2}{|c|}{$\%$ of Female gender } & \multicolumn{2}{|c|}{ Number of comorbidities } & \multicolumn{2}{|l|}{ Age } & \multicolumn{2}{|l|}{$\begin{array}{l}\text { Number of } \\
\text { symptoms }\end{array}$} \\
\hline & $\begin{array}{l}\text { Coefficient } \\
\text { (Cl 95\%) }\end{array}$ & $P$ value & $\begin{array}{l}\text { Coefficient } \\
\text { (Cl 95\%) }\end{array}$ & $\begin{array}{c}P \\
\text { value }\end{array}$ & $\begin{array}{l}\text { Coefficient } \\
\text { (Cl 95\%) }\end{array}$ & $P$ value & $\begin{array}{l}\text { Coefficient } \\
\text { (Cl 95\%) }\end{array}$ & $\begin{array}{c}P \\
\text { value }\end{array}$ \\
\hline PACS & $-0.0035(-0.0122-0.0052)$ & 0.425 & $0.0295(-0.0111-0.0702)$ & 0.154 & $0.0016(-0.0102-0.0133)$ & 0.793 & $0.0207(0.0021-0.0394)$ & 0.030 \\
\hline Body aches & $0.0075(0.0014 ; 0.0137)$ & 0.017 & - & - & $0.0038(-0.0138 ; 0.0214)$ & 0.673 & $0.0966(-0.0991 ; 0.2923)$ & 0.333 \\
\hline $\begin{array}{l}\text { Loss of } \\
\text { appetite }\end{array}$ & $-0.0022(-0.0114 ; 0.0070)$ & 0.635 & $0.0024(-0.0075 ; 0.0122)$ & 0.633 & $0.0026(-0.0013 ; 0.0065)$ & 0.194 & $0.0007(-0.0218 ; 0.0232)$ & 0.952 \\
\hline Fever & $0.0081(0.0026 ; 0.0136)$ & 0.004 & $-0.0029(-0.0390 ; 0.0332)$ & 0.874 & $-0.0050(-0.0099 ;-0.0000)$ & 0.049 & $0.0240(-0.0057 ; 0.0538)$ & 0.113 \\
\hline $\begin{array}{l}\text { Ageusia or } \\
\text { hypogeusia }\end{array}$ & $0.0066(-0.0125 ; 0.0256)$ & 0.499 & $0.0026(-0.0186 ; 0.0239)$ & 0.809 & $-0.0066(-0.0342 ; 0.0209)$ & 0.636 & $0.0172(-0.0093 ; 0.0437)$ & 0.203 \\
\hline Dysgeusia & $0.0008(-0.0137 ; 0.0154)$ & 0.912 & - & - & $0.0114(-0.0082 ; 0.0309)$ & 0.254 & $0.0205(-0.0058 ; 0.0468)$ & 0.127 \\
\hline Cephalea & $0.0078(-0.0017 ; 0.0173)$ & 0.108 & $-0.011(-0.0315 ; 0.0095)$ & 0.292 & $-0.0061(-0.0136 ; 0.0013)$ & 0.107 & $-0.0043(-0.0237 ; 0.0151)$ & 0.664 \\
\hline Dyspnea & $-0.0064(-0.0188 ; 0.0060)$ & 0.312 & $0.0022(-0.0157 ; 0.0201)$ & 0.808 & $0.0128(-0.0001 ; 0.0258)$ & 0.052 & $-0.0210(0.0467 ; 0.0047)$ & 0.109 \\
\hline Cough & $0.0015(-0.0063 ; 0.0094)$ & 0.698 & $-0.0042(-0.0208 ; 0.0124)$ & 0.620 & $-0.0064(-0.0113 ;-0.0015)$ & 0.011 & $0.012(0.0005 ; 0.0236)$ & 0.041 \\
\hline Sputum & $0.0697(-0.0677 ; 0.2072)$ & 0.320 & $-0.020(-0.112 ; 0.072)$ & 0.671 & $-0.0175(-0.0431 ; 0.0082)$ & 0.183 & $0.0399(-0.1094 ; 0.1893)$ & 0.600 \\
\hline Rhinitis* & $-0.0093(-0.0459 ; 0.0273)$ & 0.619 & - & - & $-0.0079(-0.0176 ; 0.0018)$ & 0.110 & $0.0038(-0.0360 ; 0.0435)$ & 0.853 \\
\hline
\end{tabular}




\begin{tabular}{|c|c|c|c|c|c|c|c|c|}
\hline Red eye ${ }^{\star *}$ & $-0.0230(-0.0528 ; 0.0068)$ & 0.130 & $-0.0165(-0.0267 ;-0.0062)$ & 0.002 & $-0.1093(-0.3159 ; 0.0973)$ & 0.300 & $-0.0151(-0.0278 ;-0.0025)$ & 0.019 \\
\hline Sleep disorders & $-0.0057(-0.0179 ; 0.0065)$ & 0.361 & $-0.0065(-0.0216 ; 0.0085)$ & 0.394 & $0.0067(-0.0076 ; 0.0209)$ & 0.357 & $-0.0139(-0.0733 ; 0.0455)$ & 0.646 \\
\hline Memory loss & $0.0017(-0.0207 ; 0.0240)$ & 0.8846 & - & - & $0.0012(-0.0264 ; 0.0289)$ & 0.931 & $0.1041(-0.2078 ; 0.4160)$ & 0.513 \\
\hline
\end{tabular}

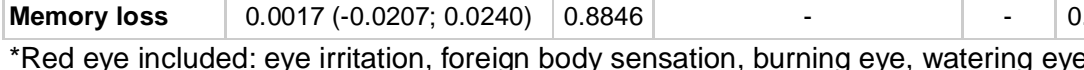

${ }^{*}$ Rhinitis included: congested nose and runny nose. 
Supplementary figures. Forest plots of long-term manifestation pooled prevalence estimates. 


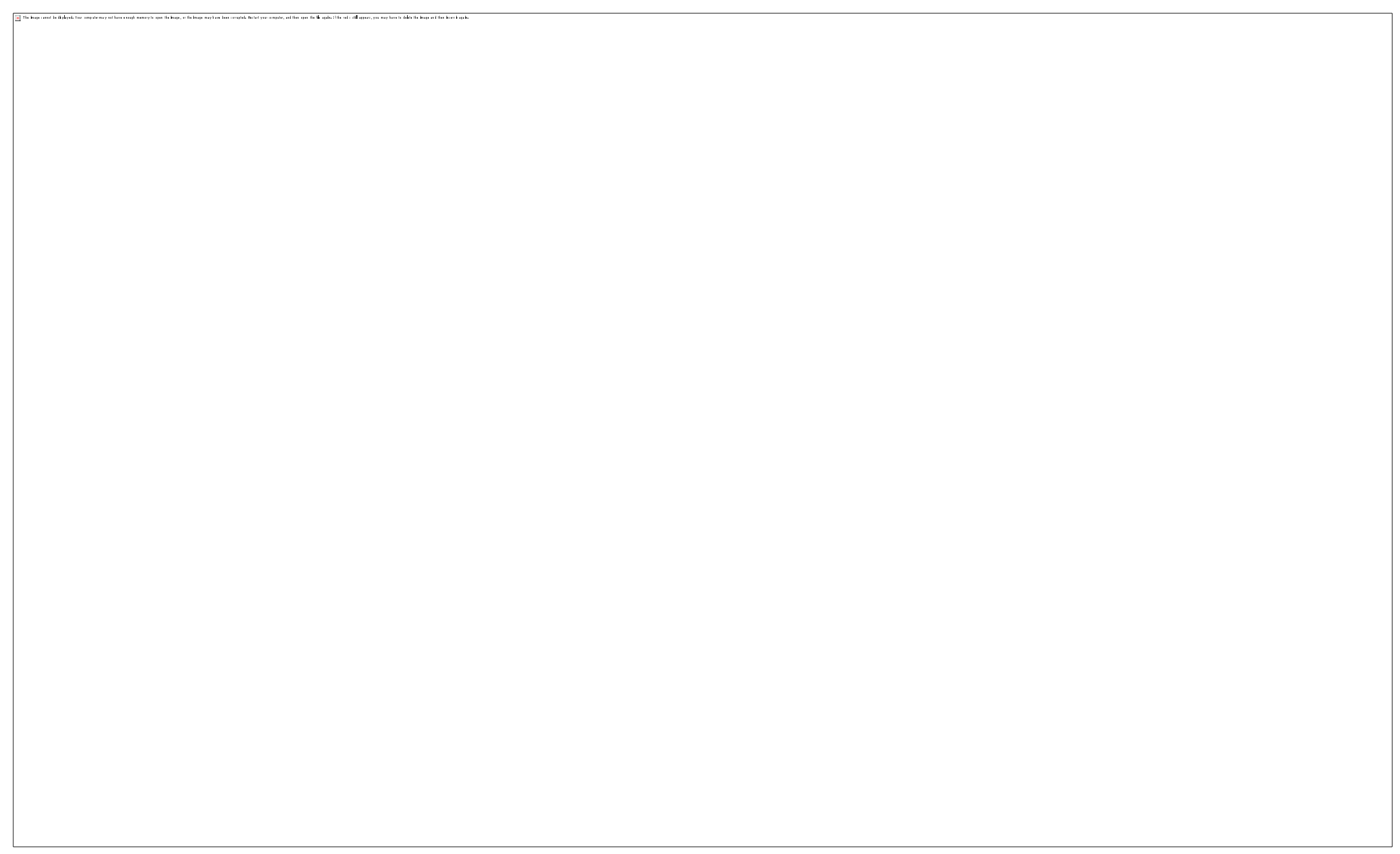




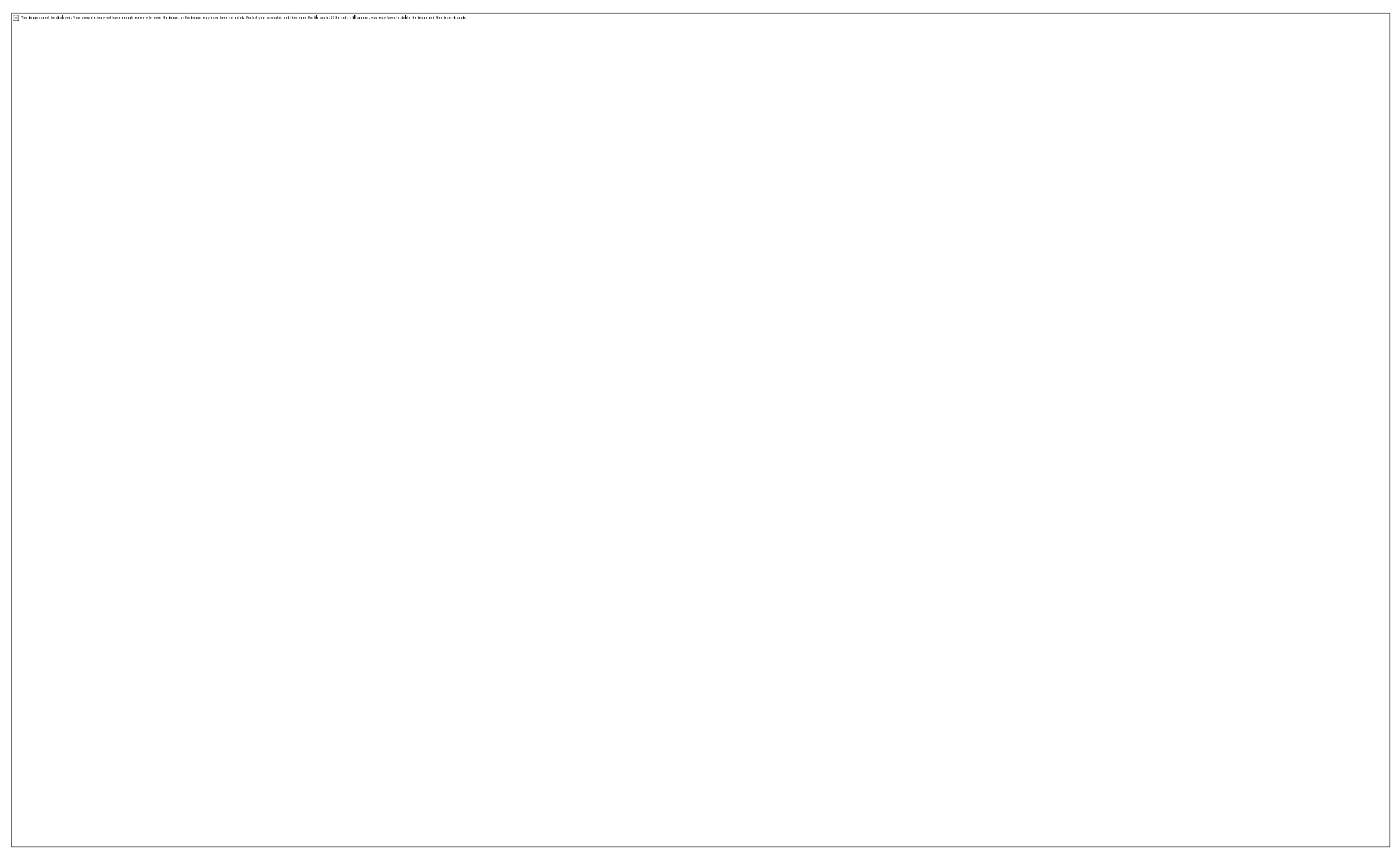




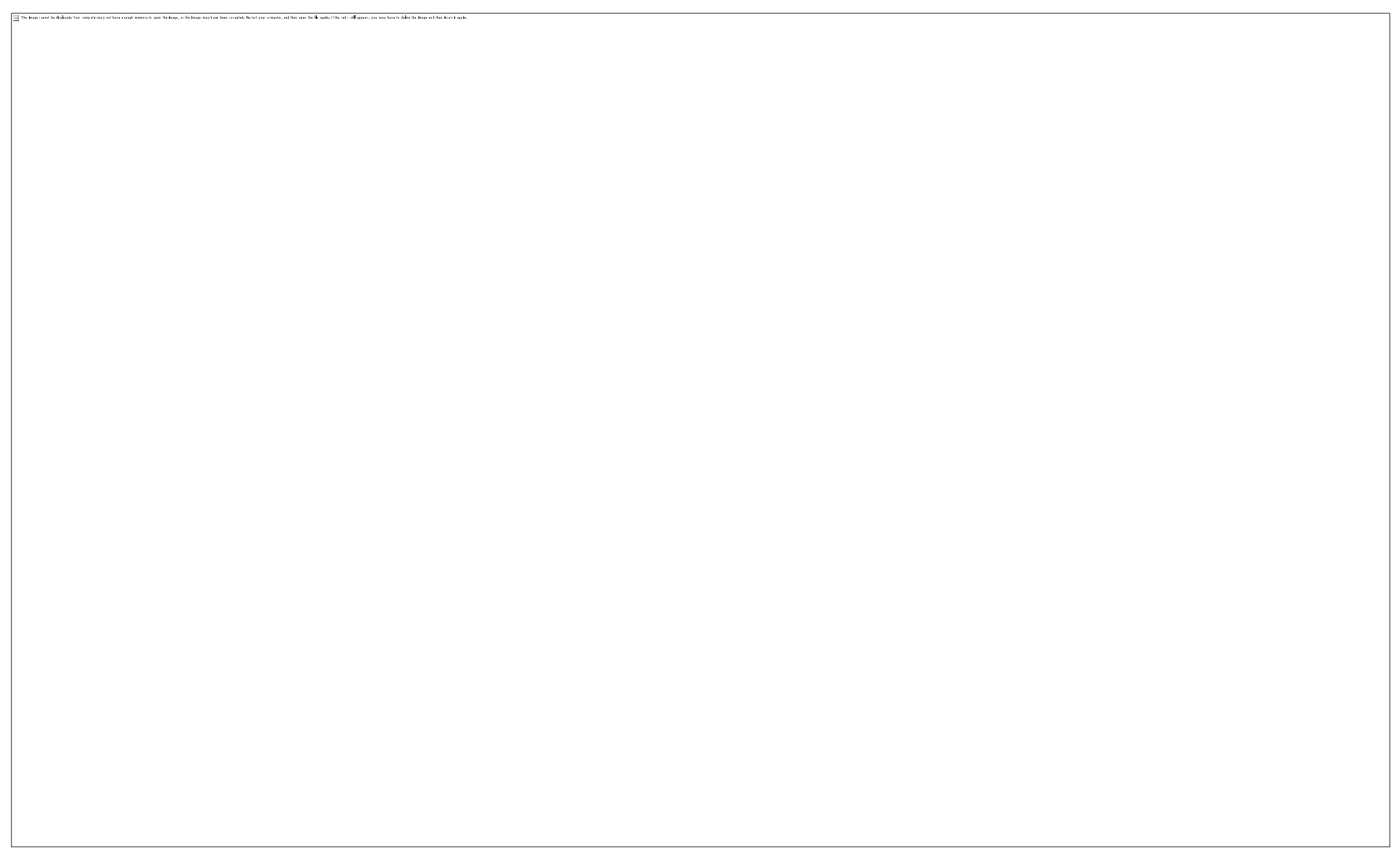




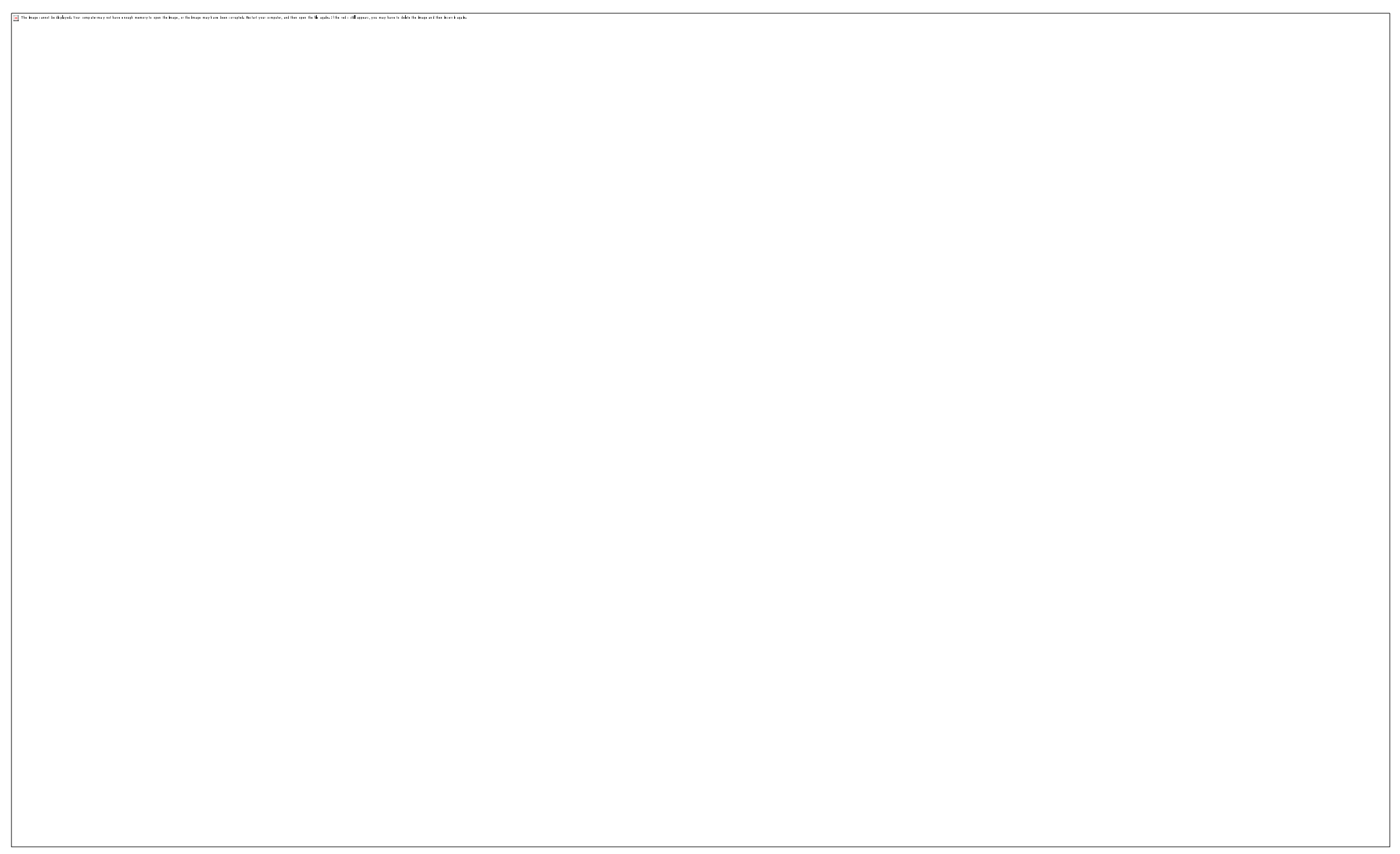




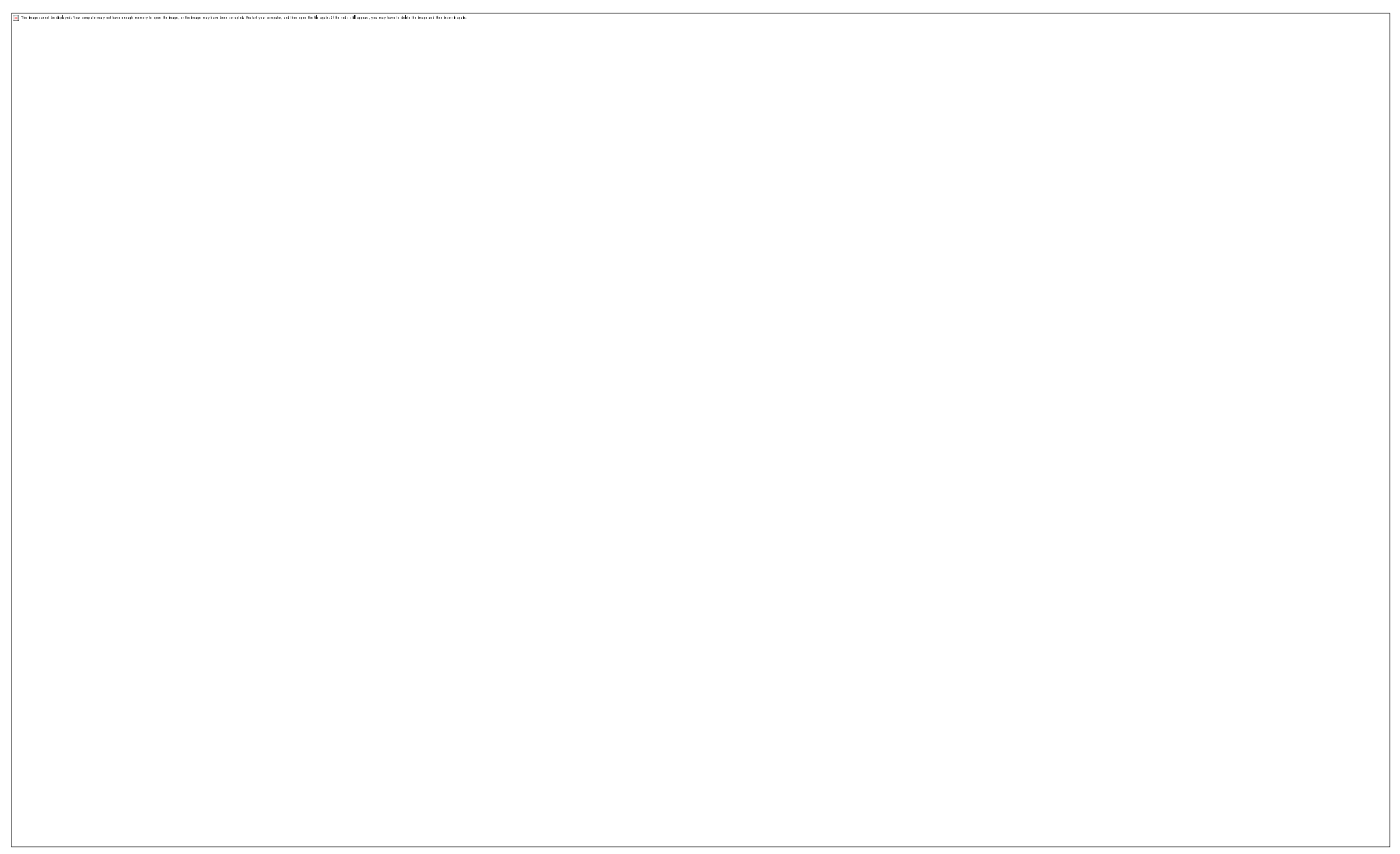




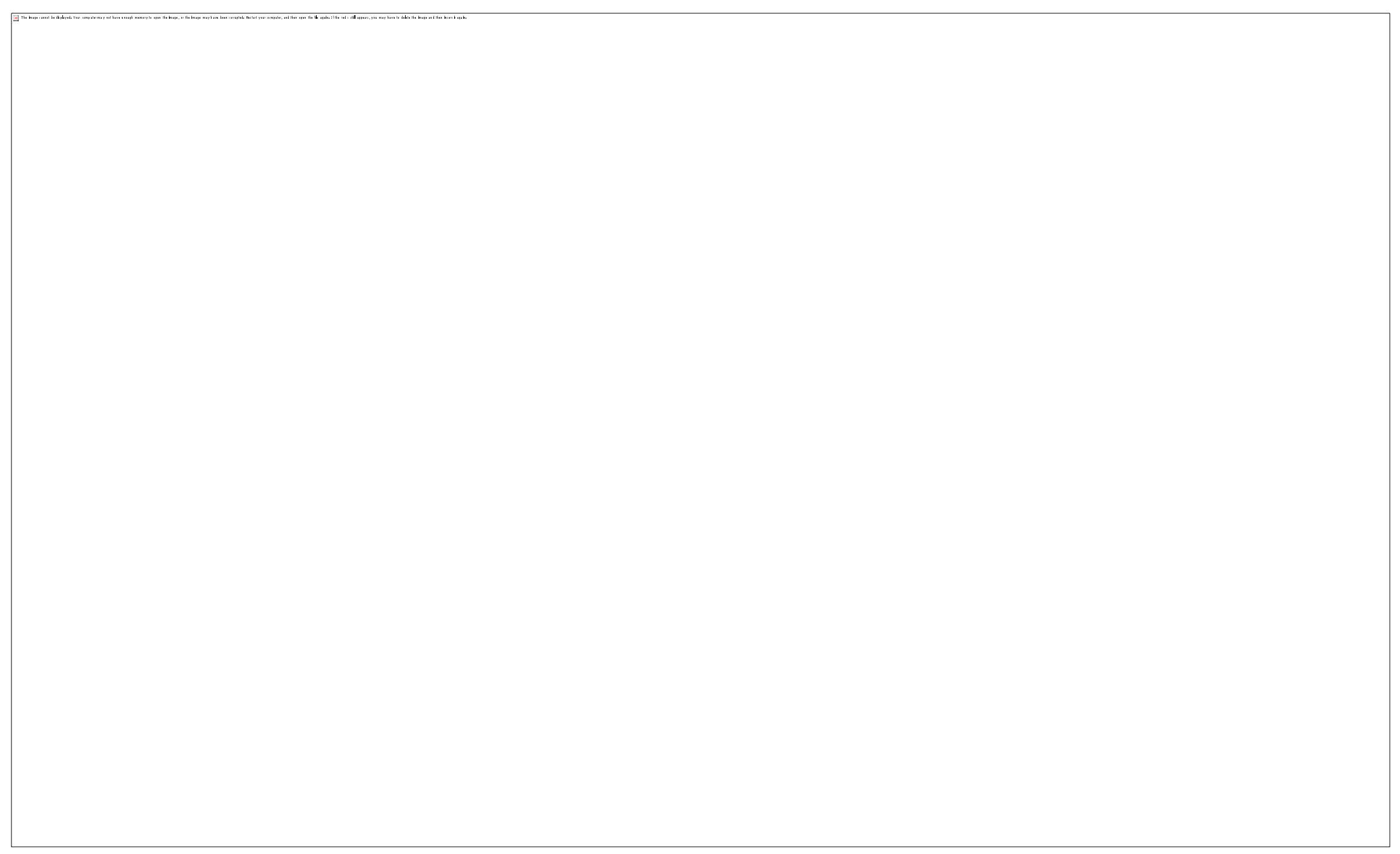




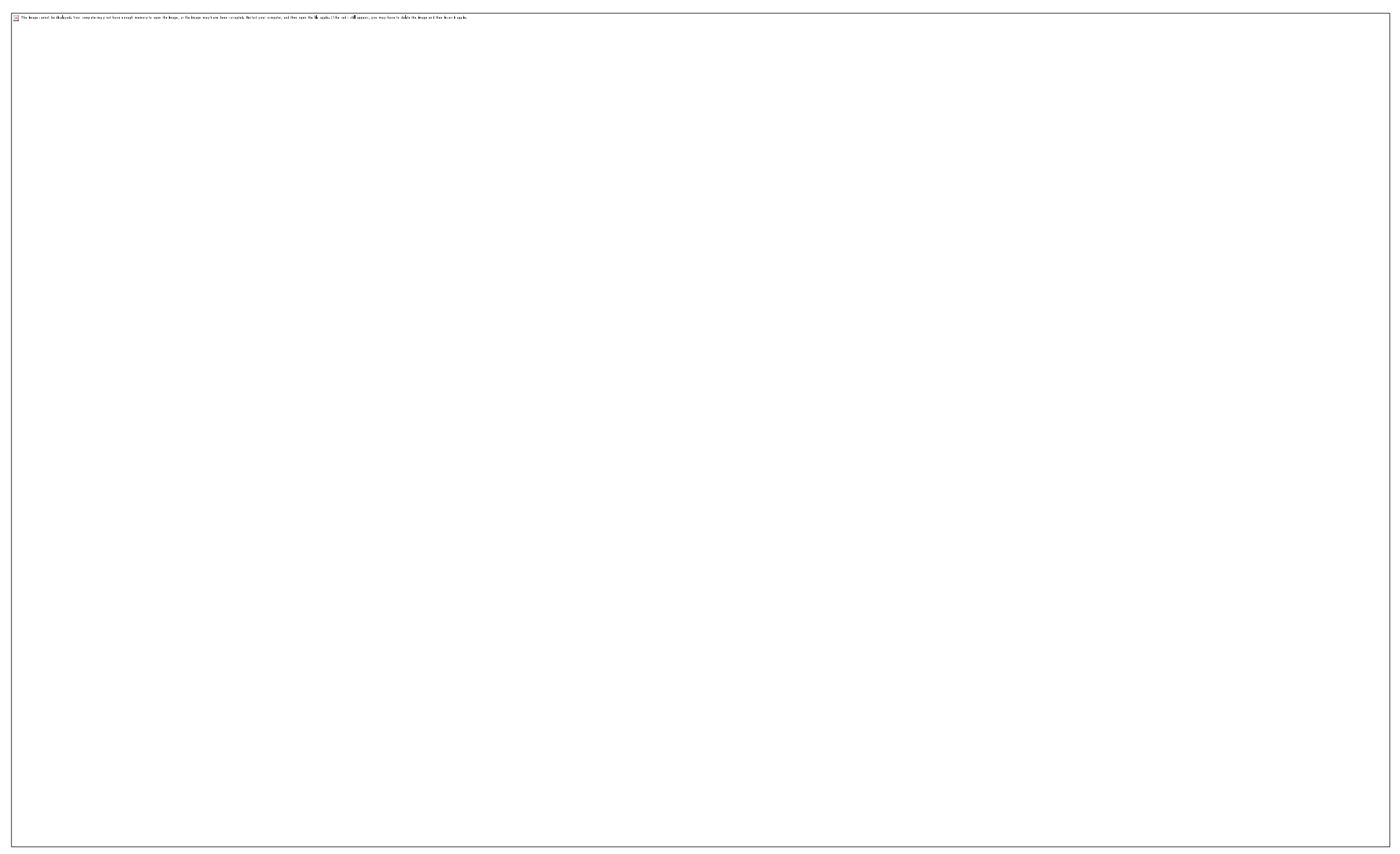




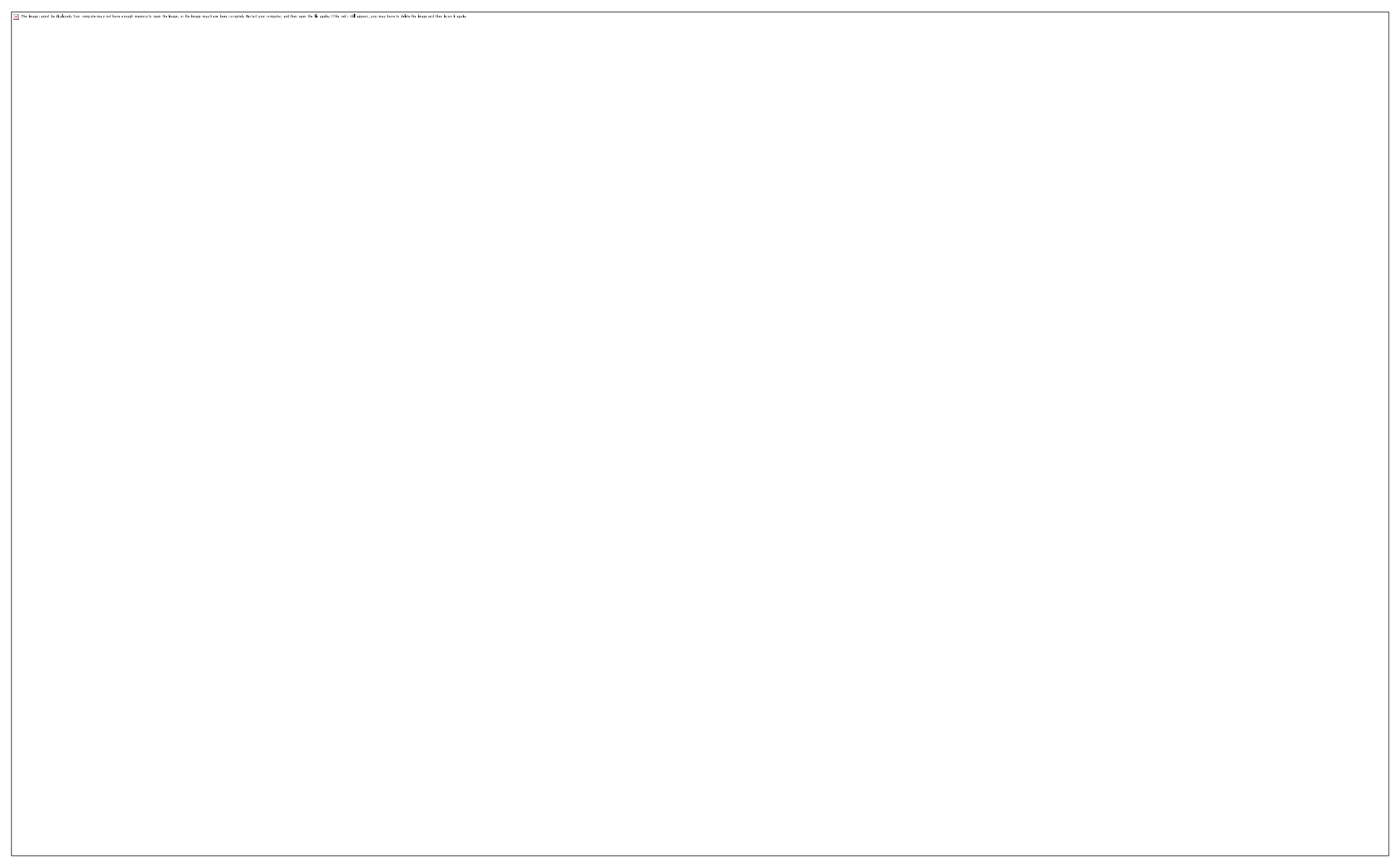




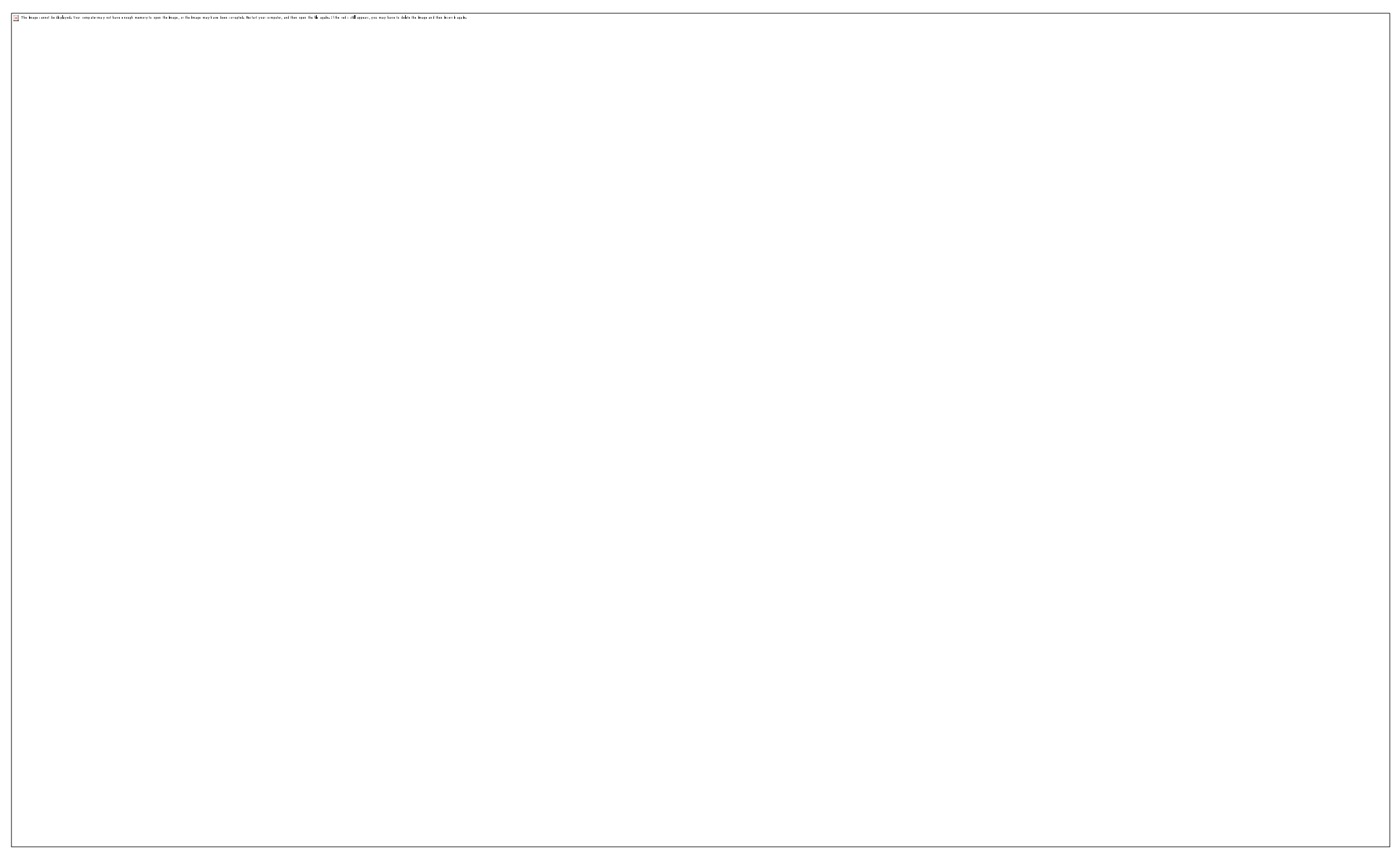




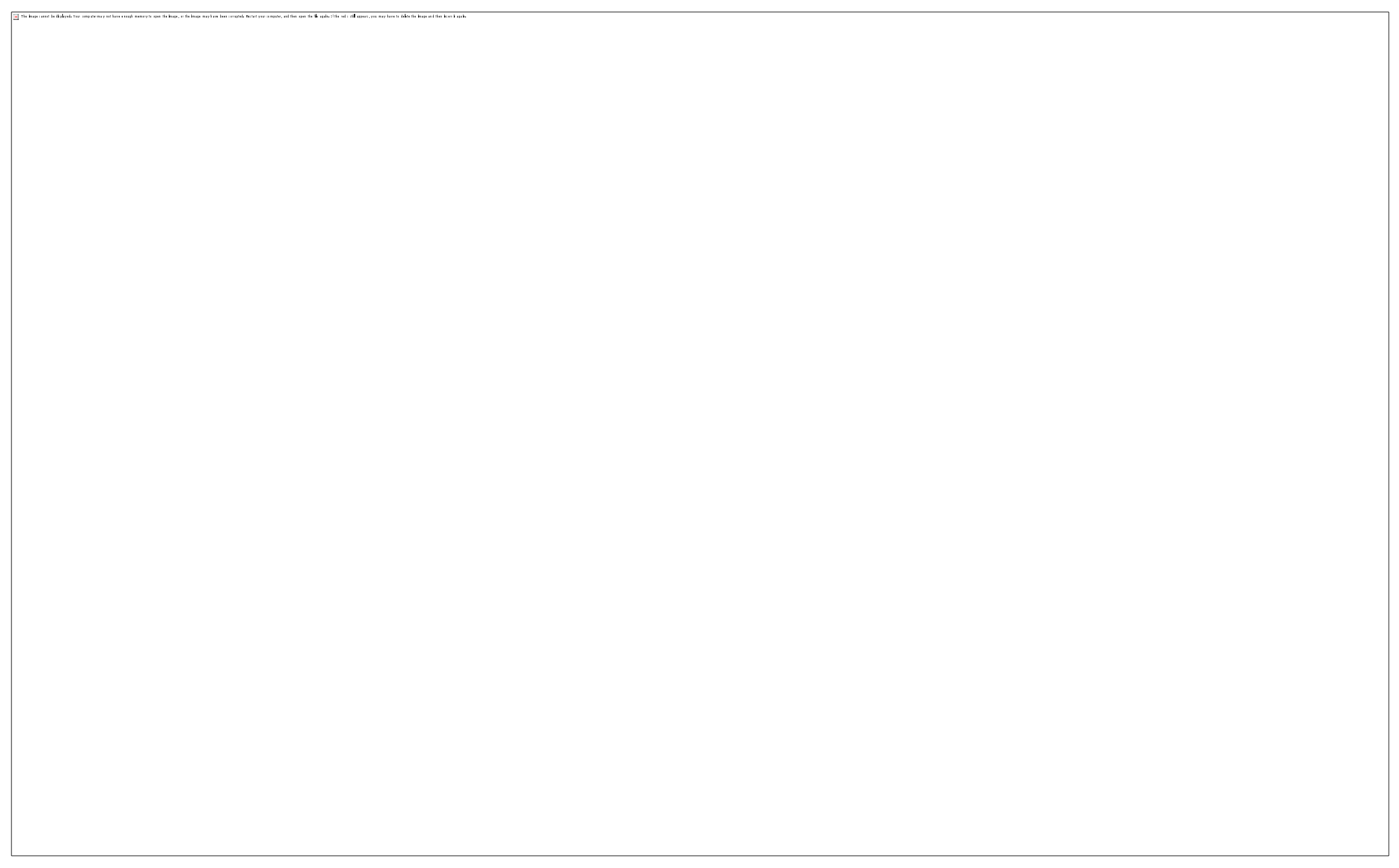




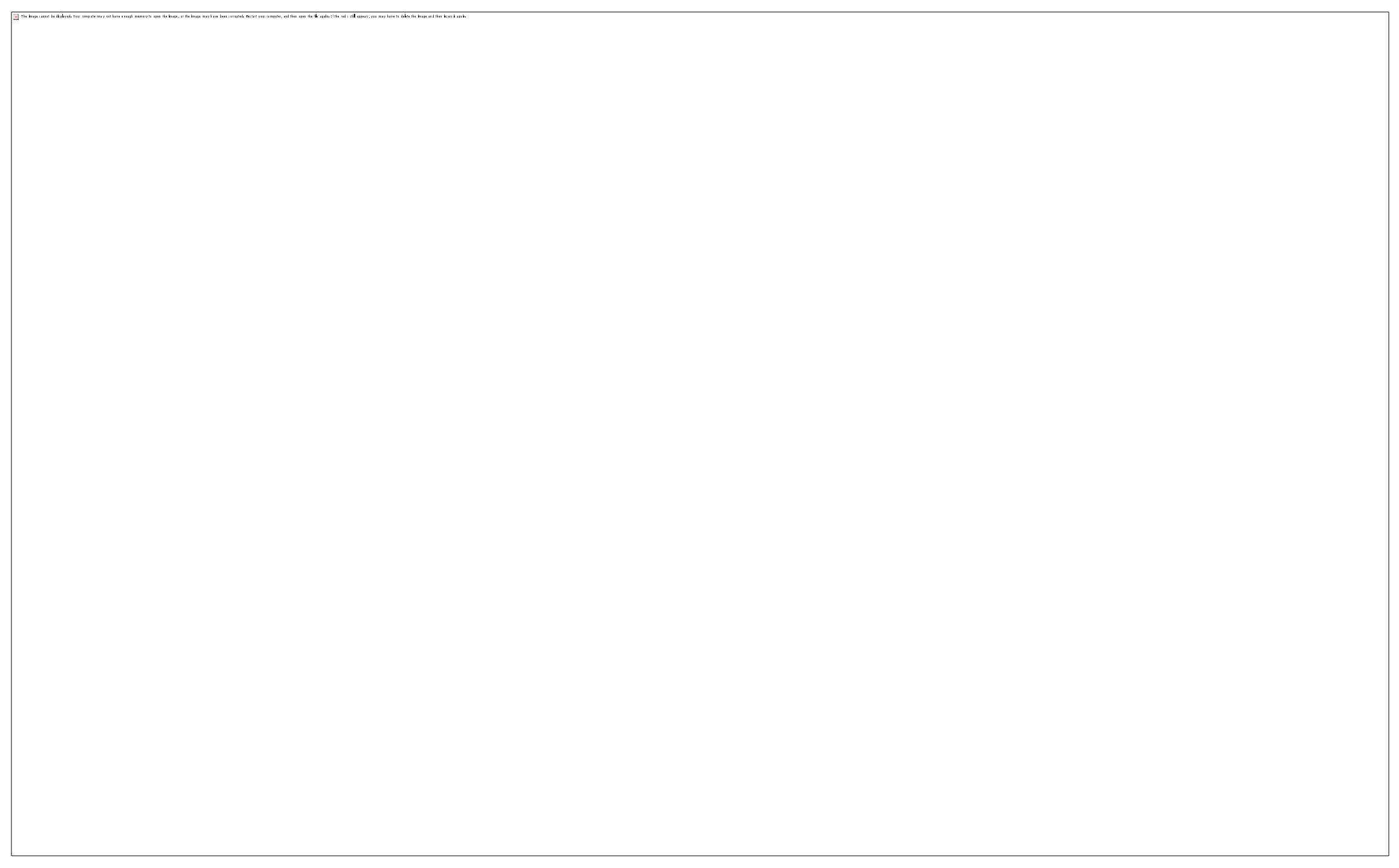




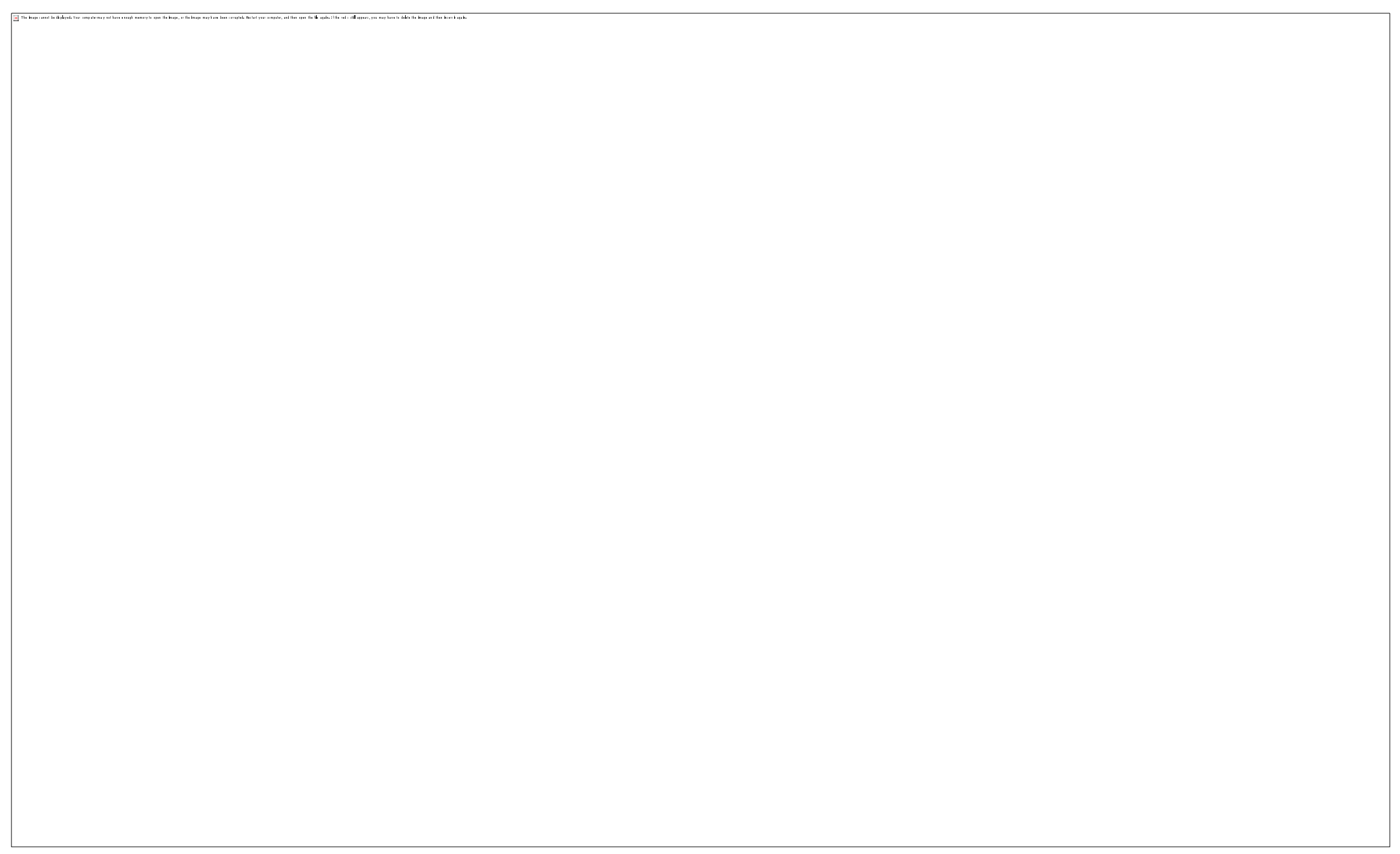




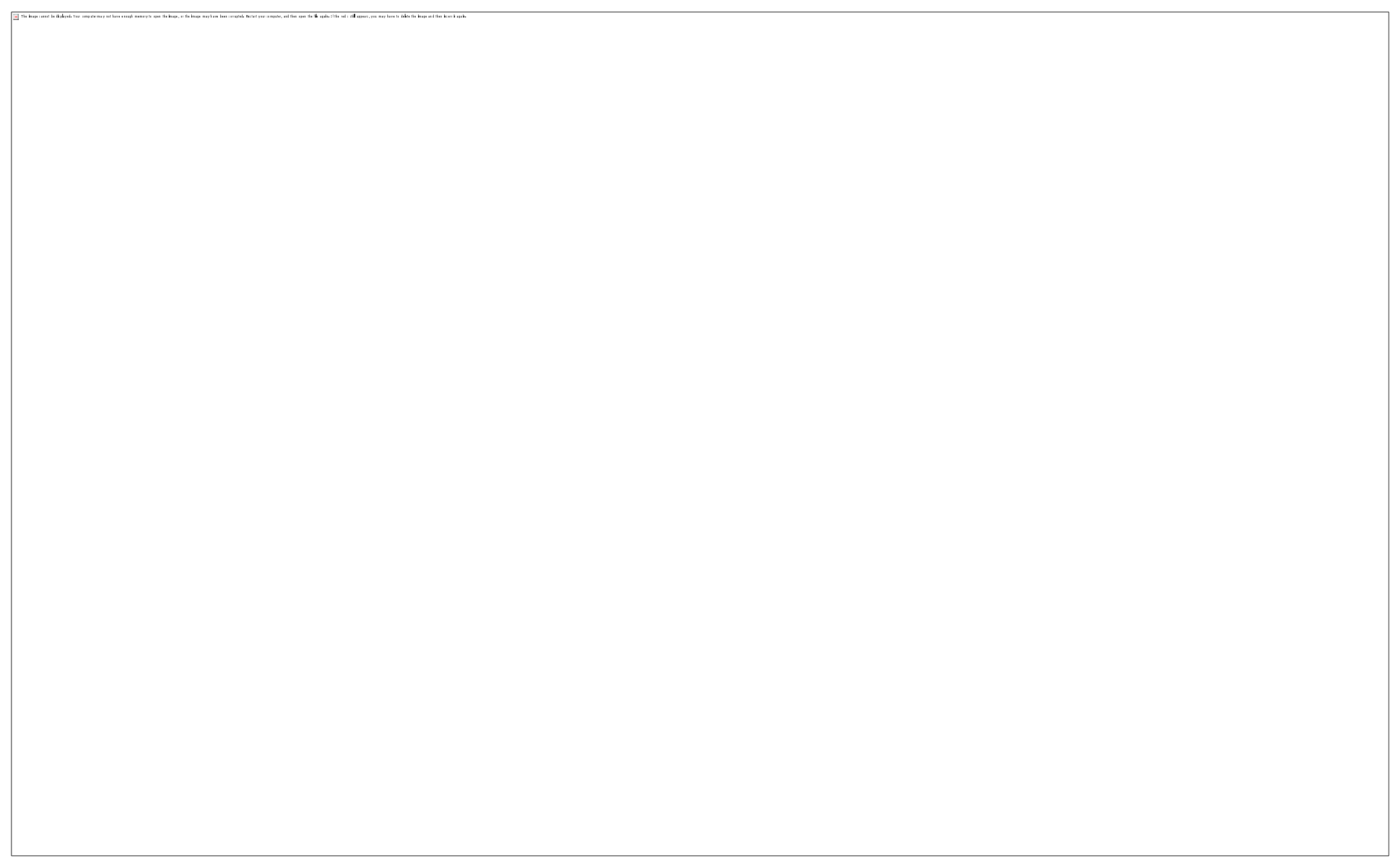




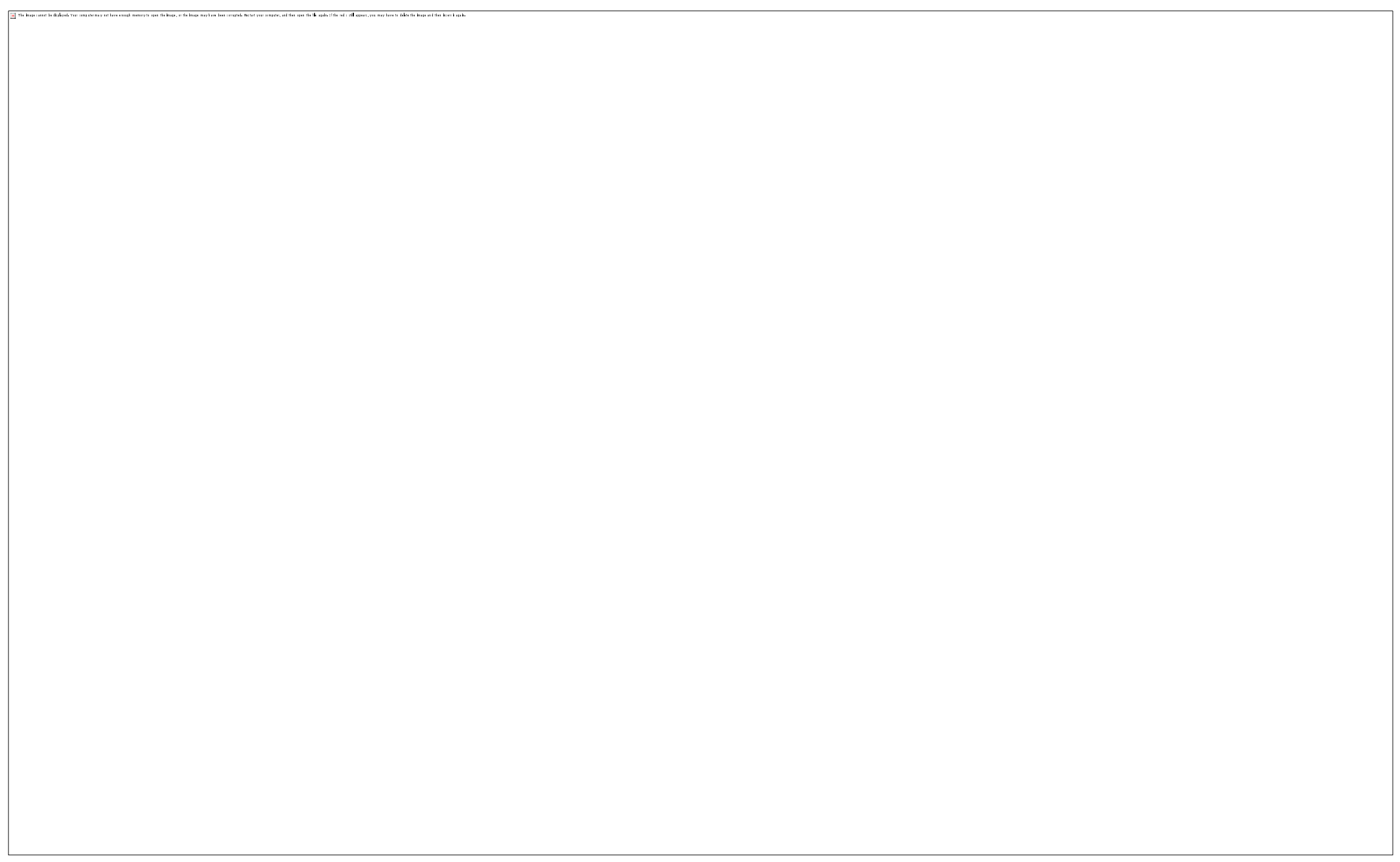




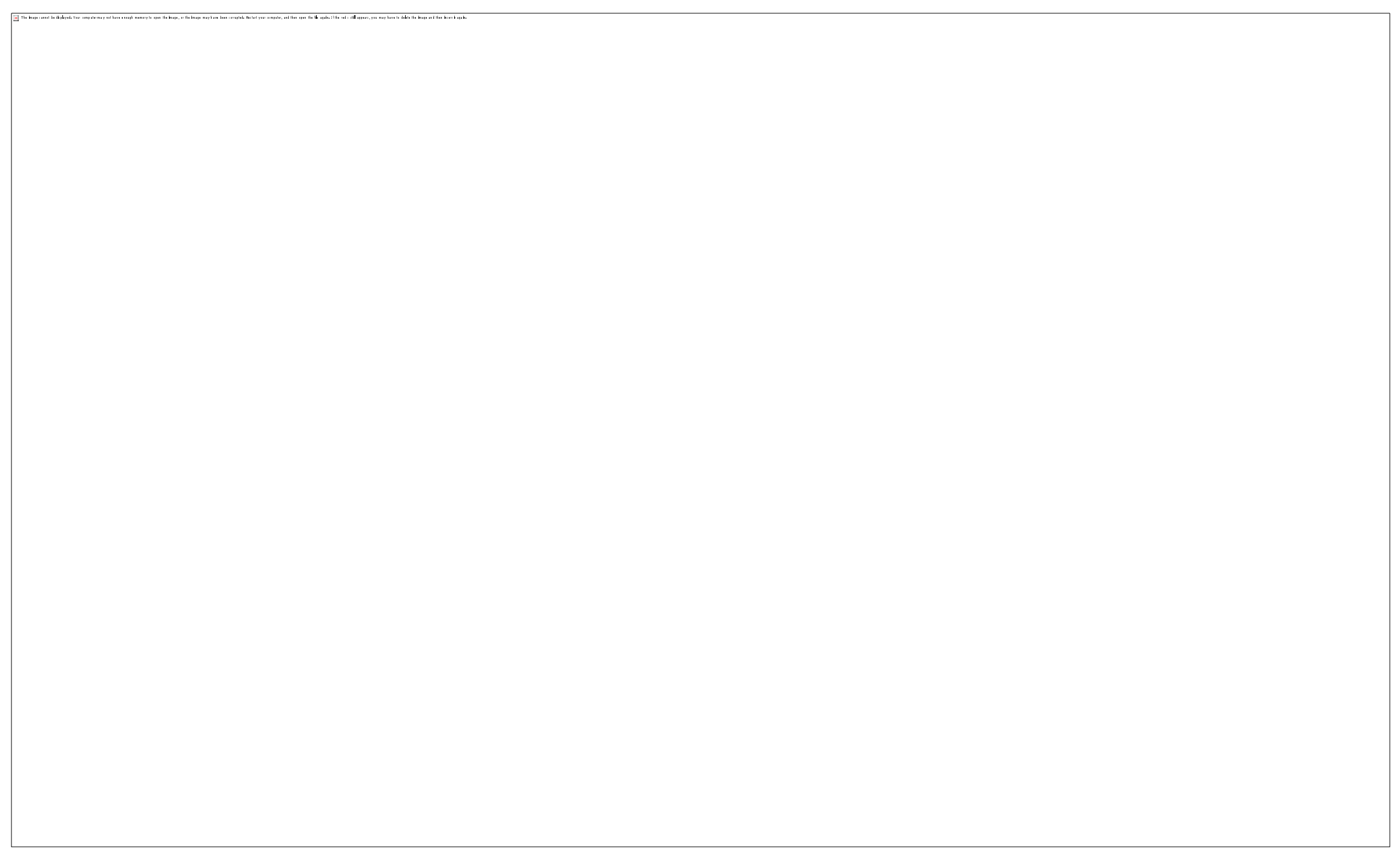




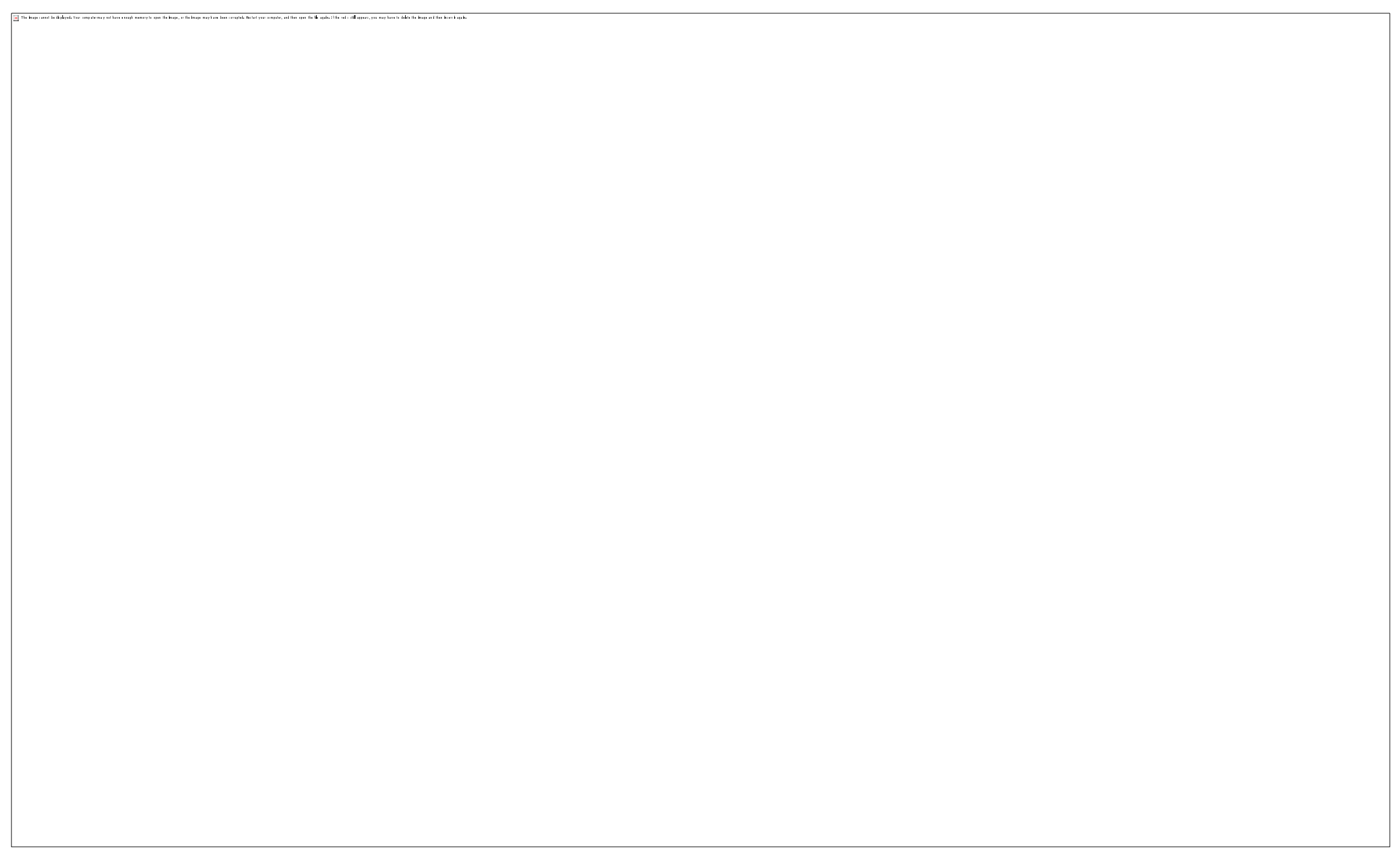




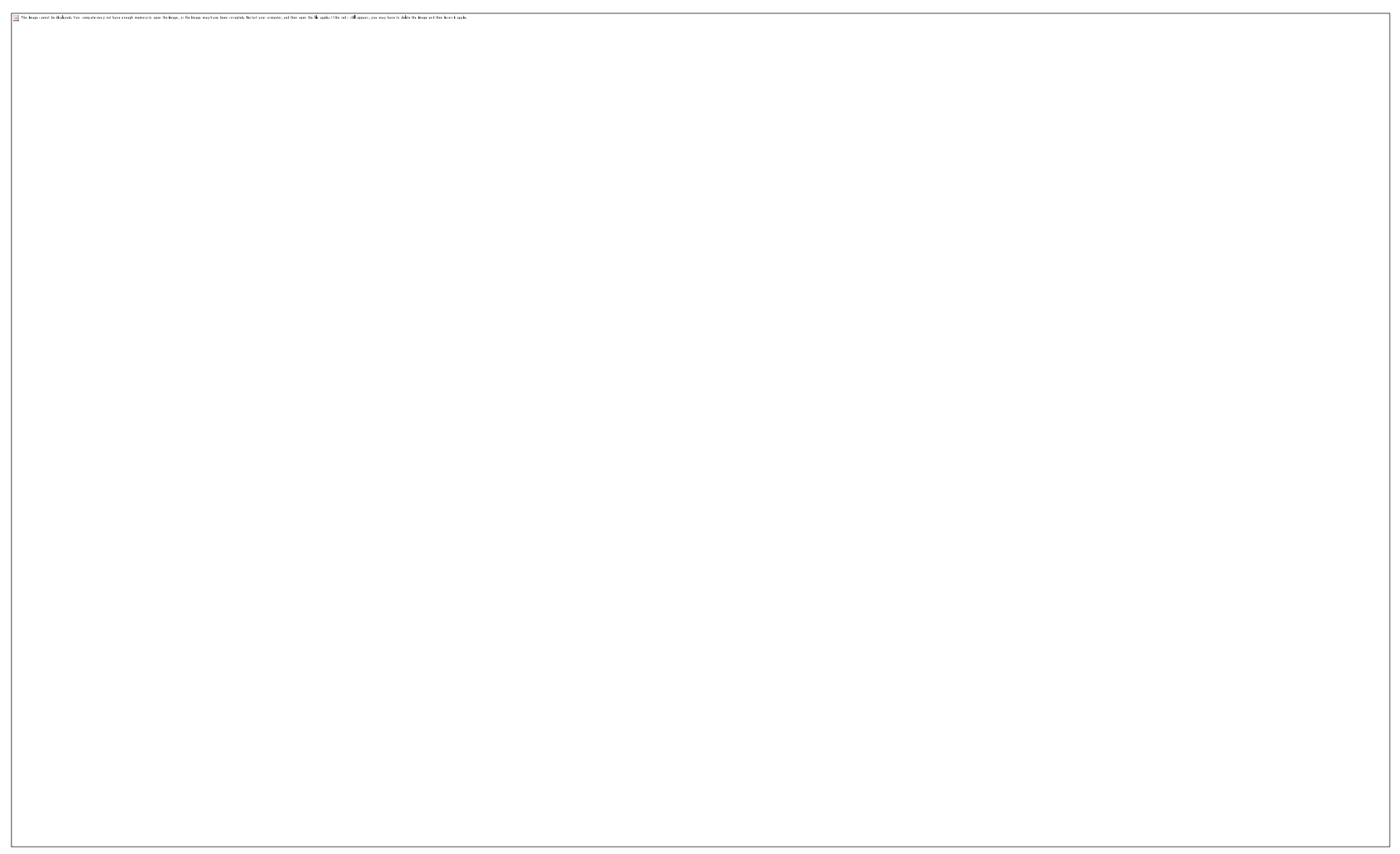




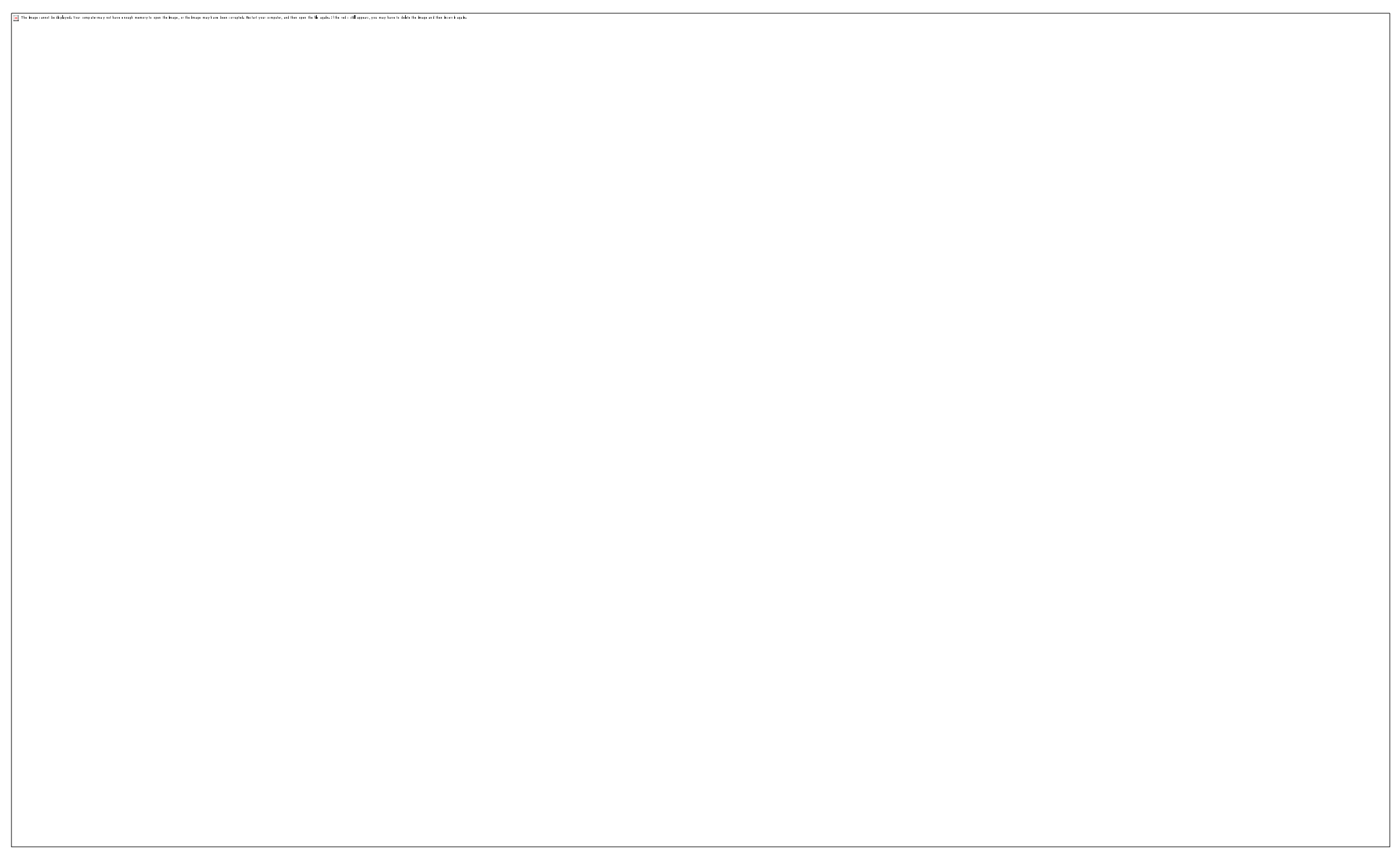




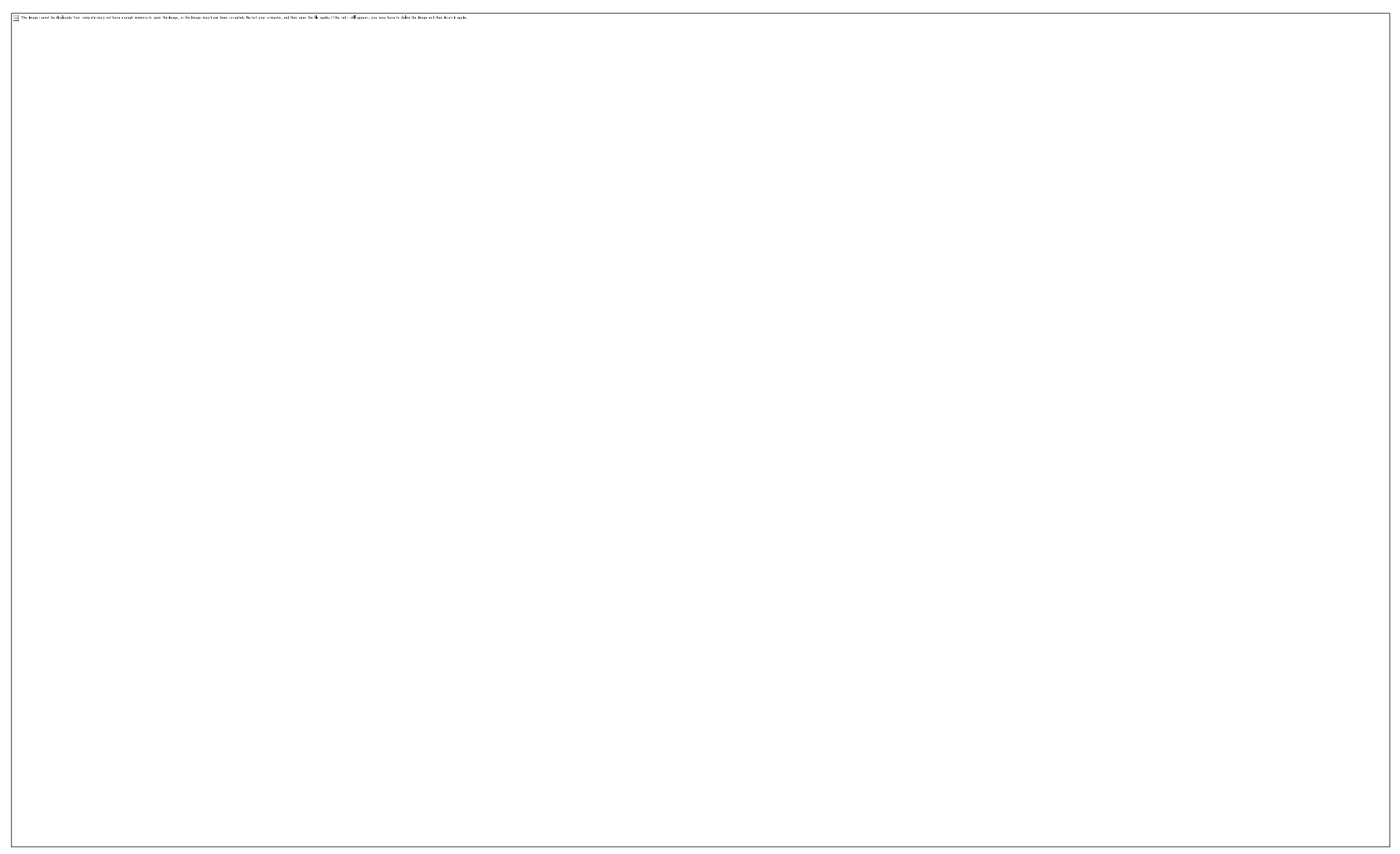




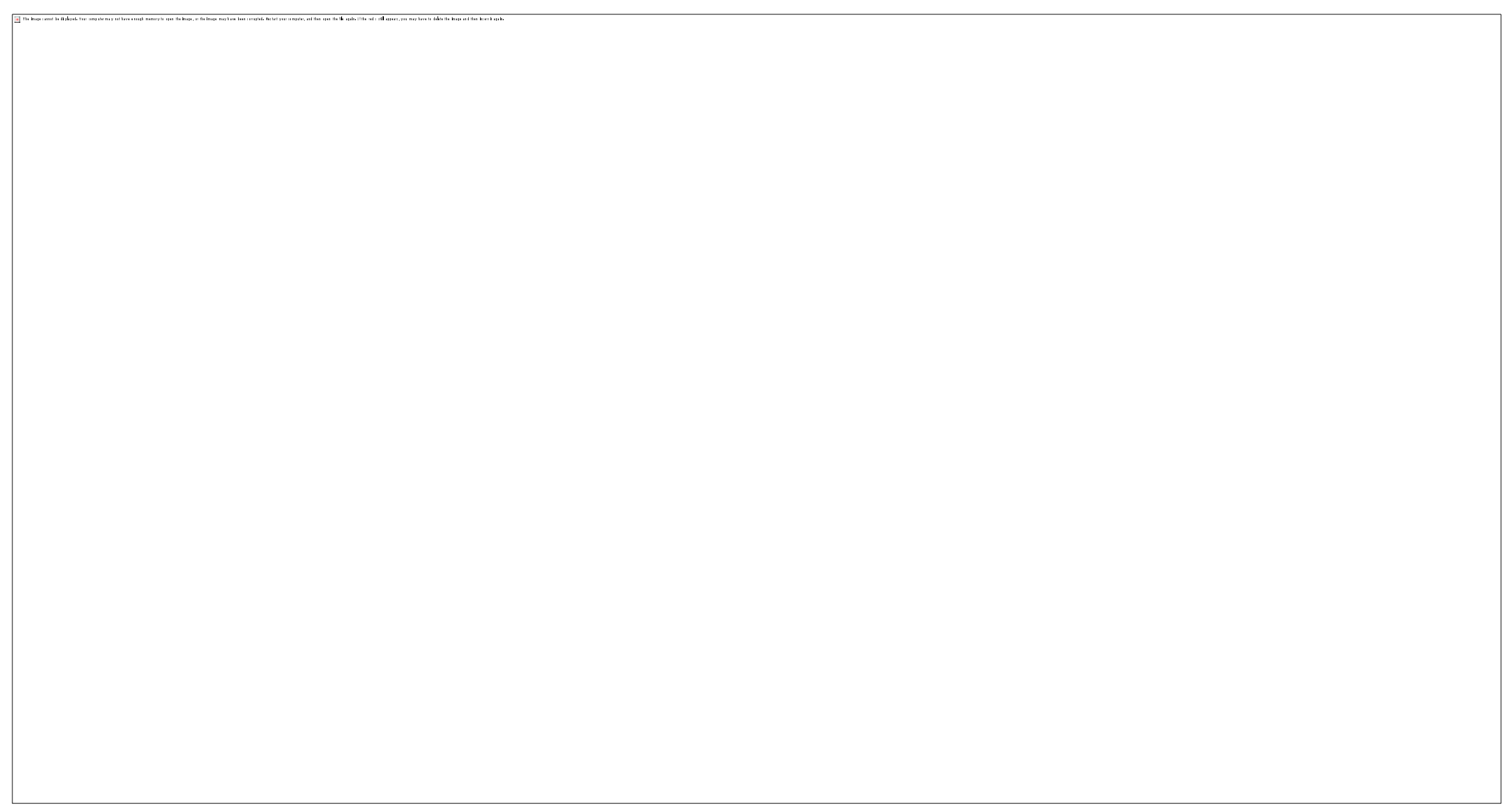




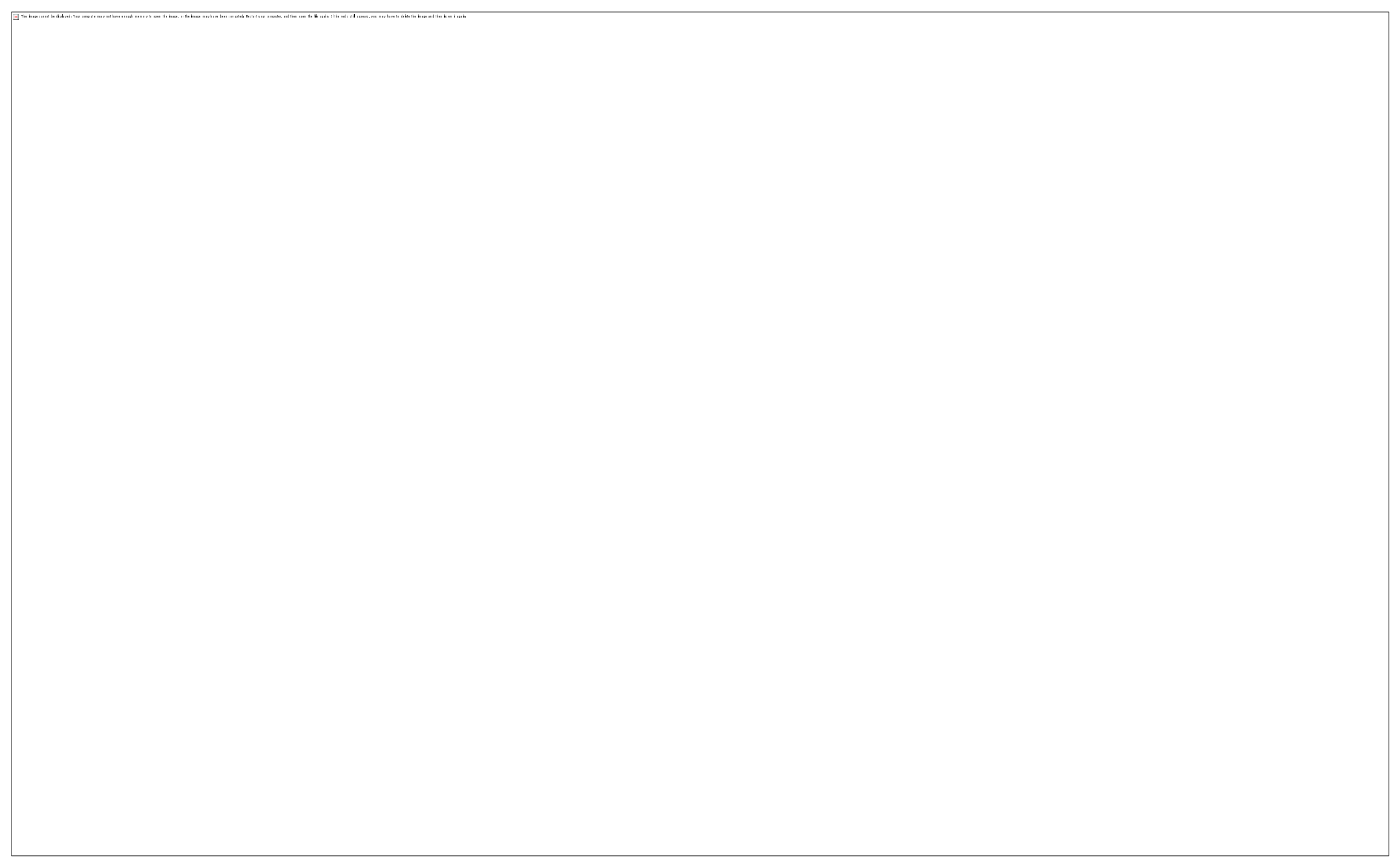




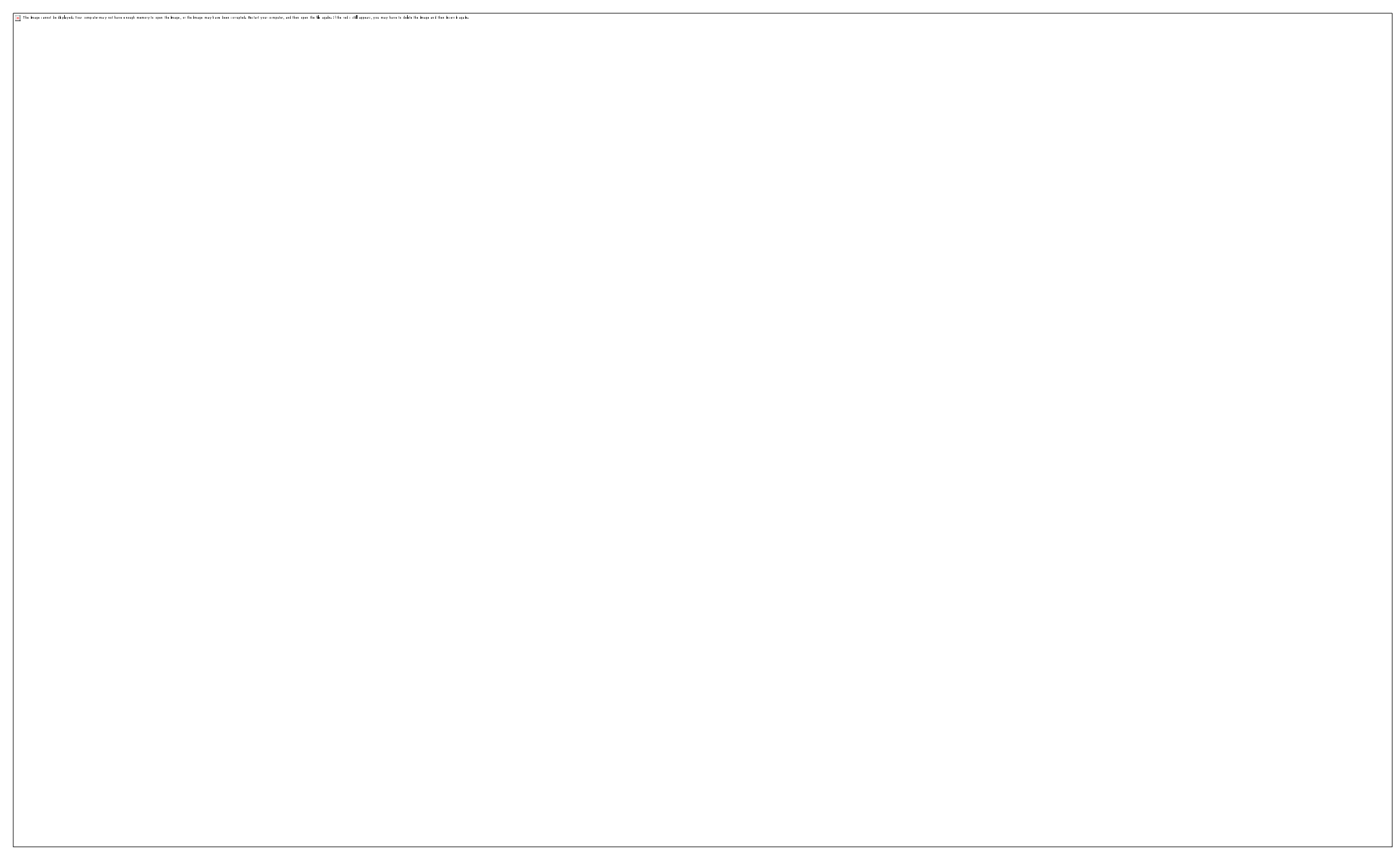




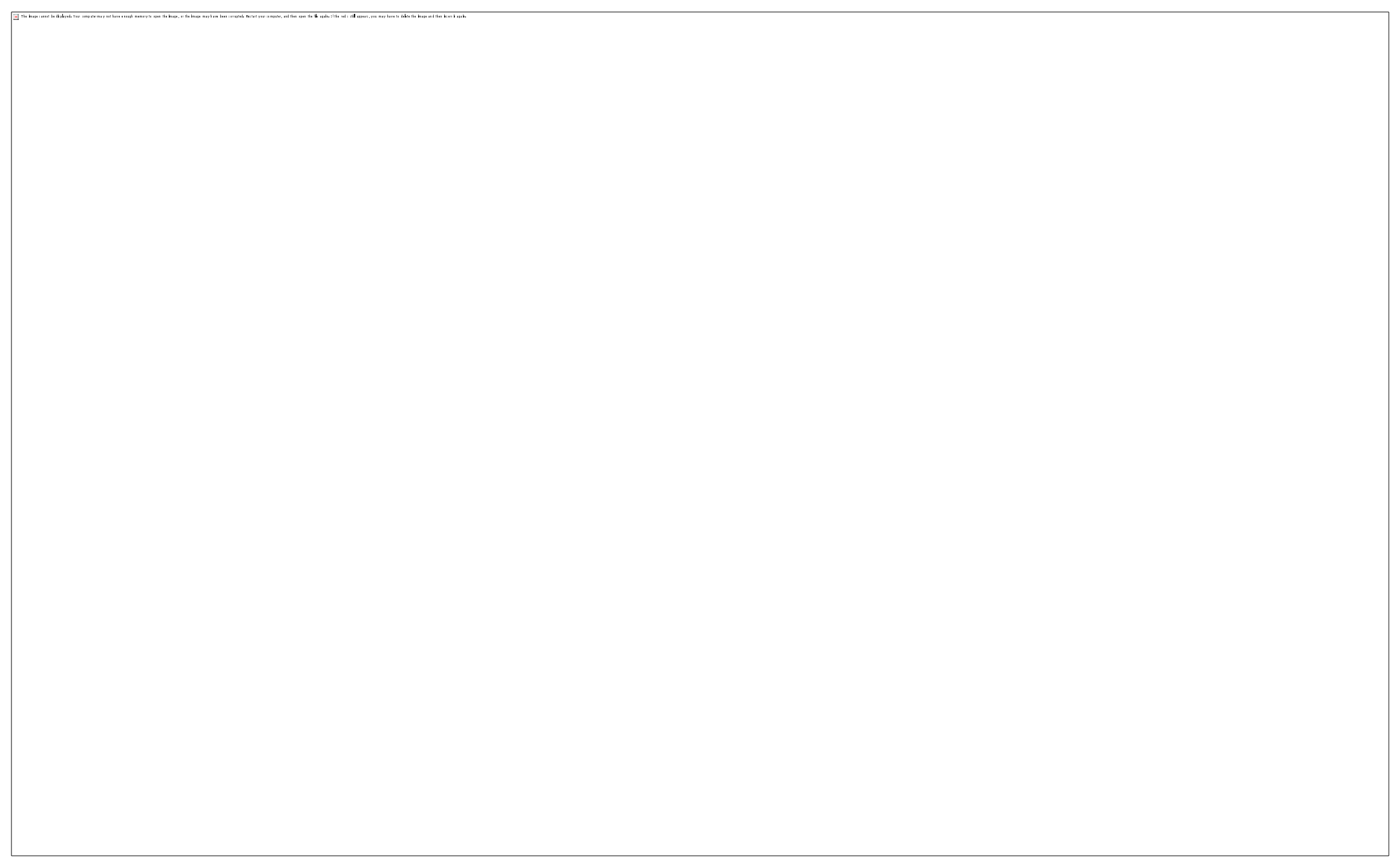




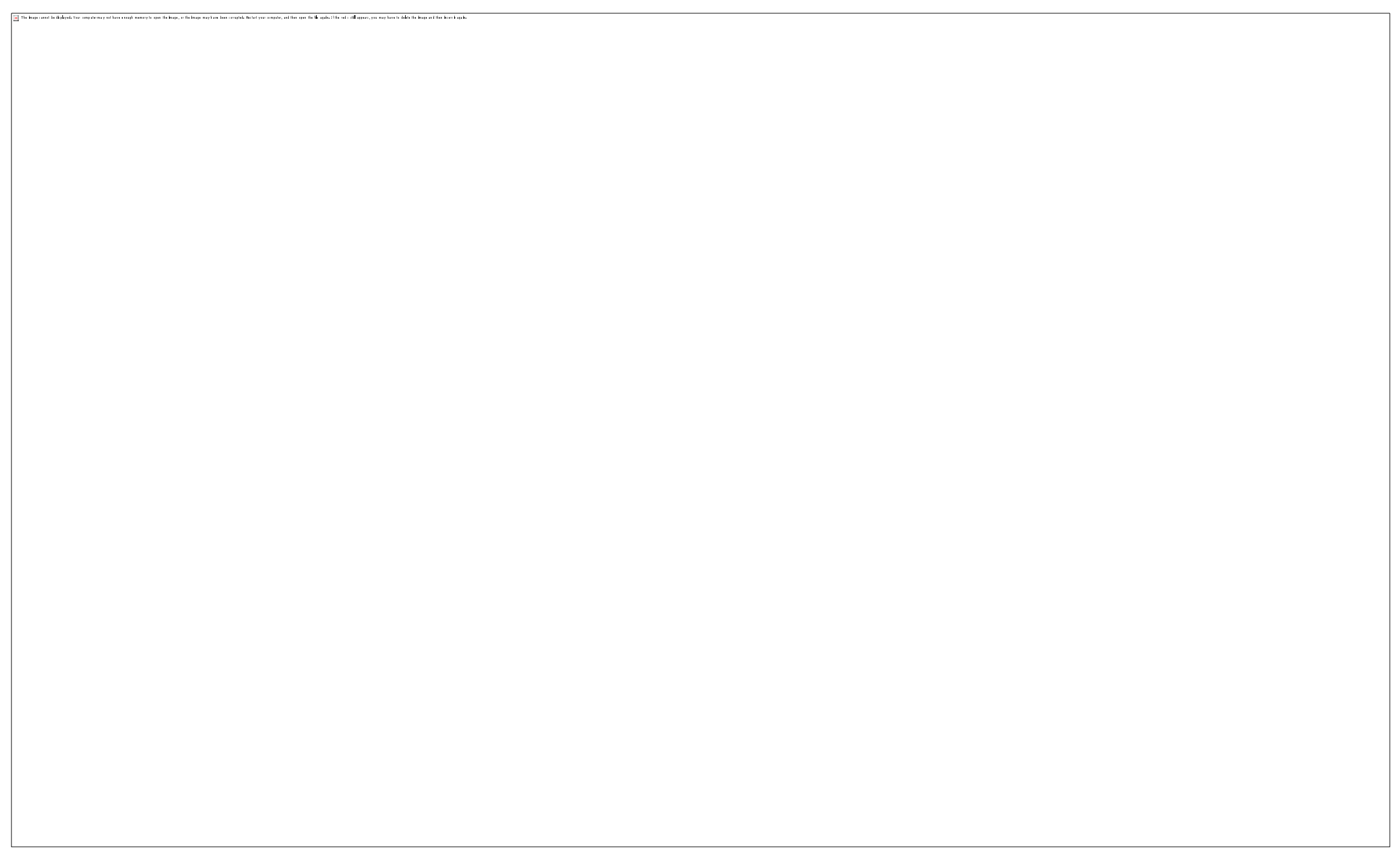




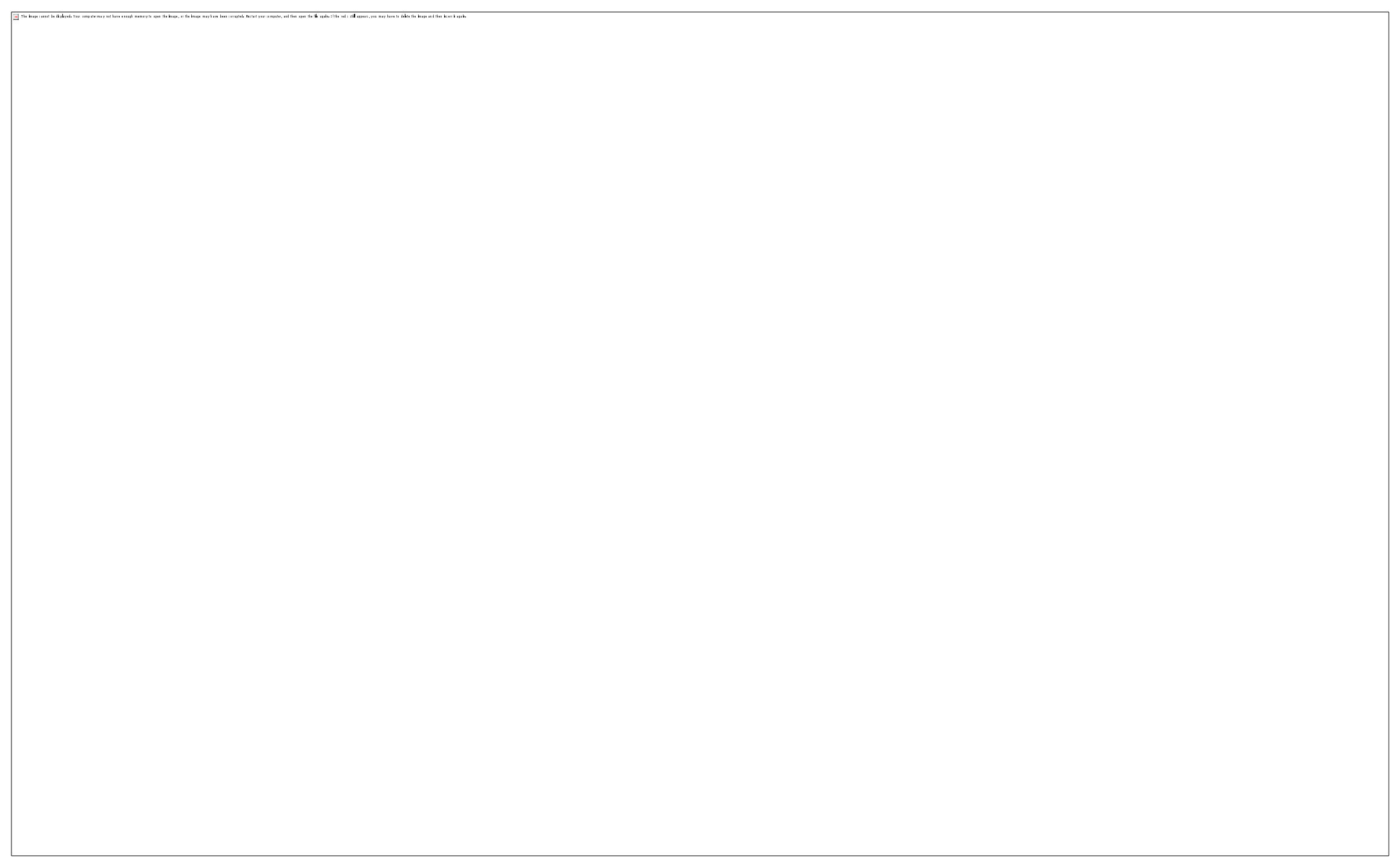




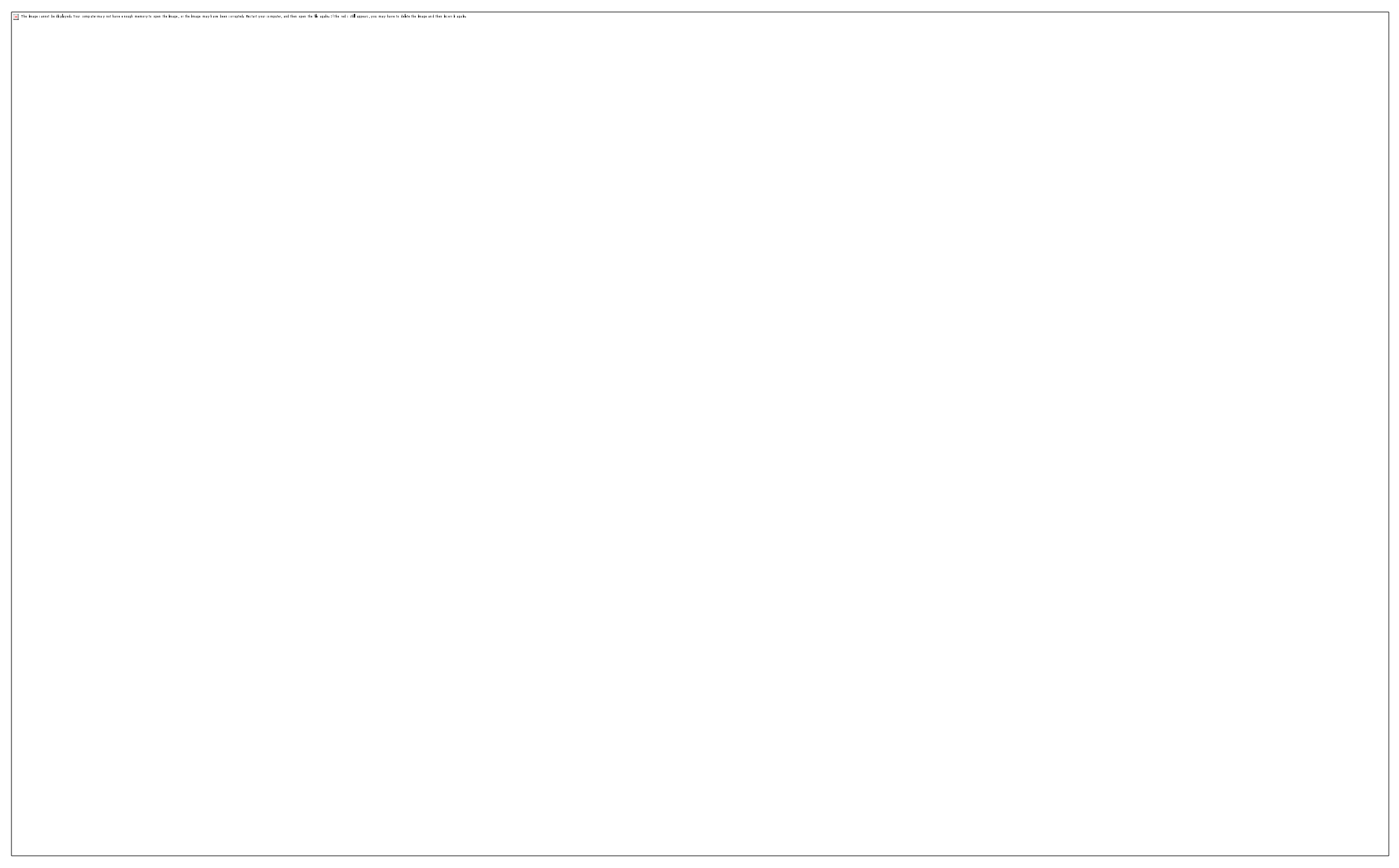




\section{Study}

Niklassen, et al.

Vaira, et al.

Tomasini, et al.

Jacobs, et al.

Park, et al.

Taboada, et al.

Garrigues, et al.

Landi, et al.

Sonnweber, et al.

Barón-Sánchez, et al. [ ]

Carvalho-Schneider, et al.

Gerkin, et al.

Fixed effect model

Random effects model

Heterogeneity: $I^{2}=99 \%, \tau^{2}=0.0892, p<0.01$

$\begin{array}{rr}1 & 93 \\ 8 & 138 \\ 6 & 105 \\ 17 & 183 \\ 4 & 46 \\ 10 & 91 \\ 16 & 120 \\ 16 & 109 \\ 26 & 145 \\ 7 & 31 \\ 40 & 150\end{array}$

15963147

4358
Events Total Anosmia and hyposmia

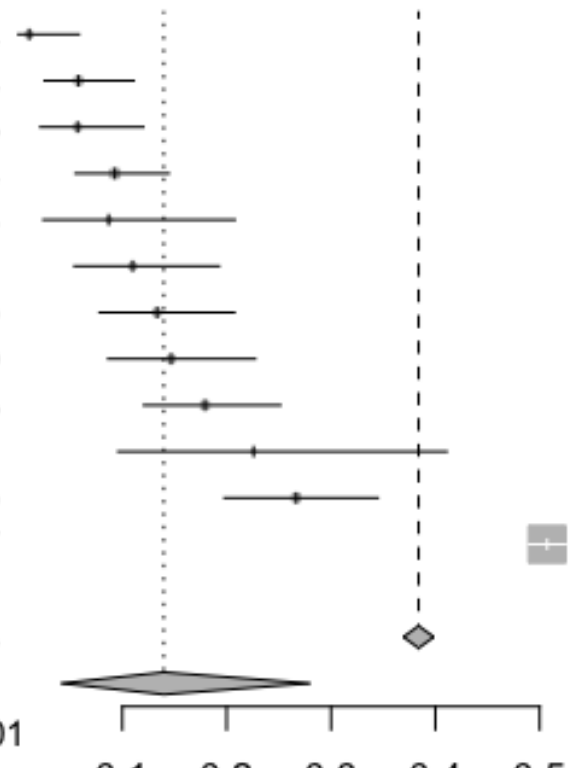

$\begin{array}{lllll}0.1 & 0.2 & 0.3 & 0.4 & 0.5\end{array}$
Weight Weight Proportion $\quad 95 \%-\mathrm{Cl}$ (fixed) (random)

\section{$0.01[0.00 ; 0.06] \quad 2.1 \% \quad 8.3 \%$}

$0.06[0.03 ; 0.11] \quad 3.2 \% \quad 8.4 \%$

$0.06[0.02 ; 0.12] \quad 2.4 \% \quad 8.4 \%$

$0.09[0.06 ; 0.14] \quad 4.2 \% \quad 8.5 \%$

$0.09[0.02 ; 0.21] \quad 1.1 \% \quad 8.1 \%$

$0.11[0.05 ; 0.19] \quad 2.1 \% \quad 8.3 \%$

$0.13[0.08 ; 0.21] \quad 2.8 \% \quad 8.4 \%$

$0.15[0.09 ; 0.23] \quad 2.5 \% \quad 8.4 \%$

$0.18[0.12 ; 0.25] \quad 3.3 \% \quad 8.4 \%$

$0.23[0.10 ; 0.41] \quad 0.7 \% \quad 7.9 \%$

$0.27[0.20 ; 0.34] \quad 3.4 \% \quad 8.4 \%$

$0.51[0.49 ; 0.52] \quad 72.1 \% \quad 8.6 \%$

$0.38[0.37 ; 0.40] 100.0 \%$ $0.14[0.04 ; 0.28] \quad--\quad 100.0 \%$ 


\section{Study}

Vaira, et al.

Jacobs, et al.

Garrigues, et al.

Carvalho-Schneider, et al.

Fixed effect model

Random effects model

Heterogeneity: $I^{2}=91 \%, \tau^{2}=0.0169, p<0.01$

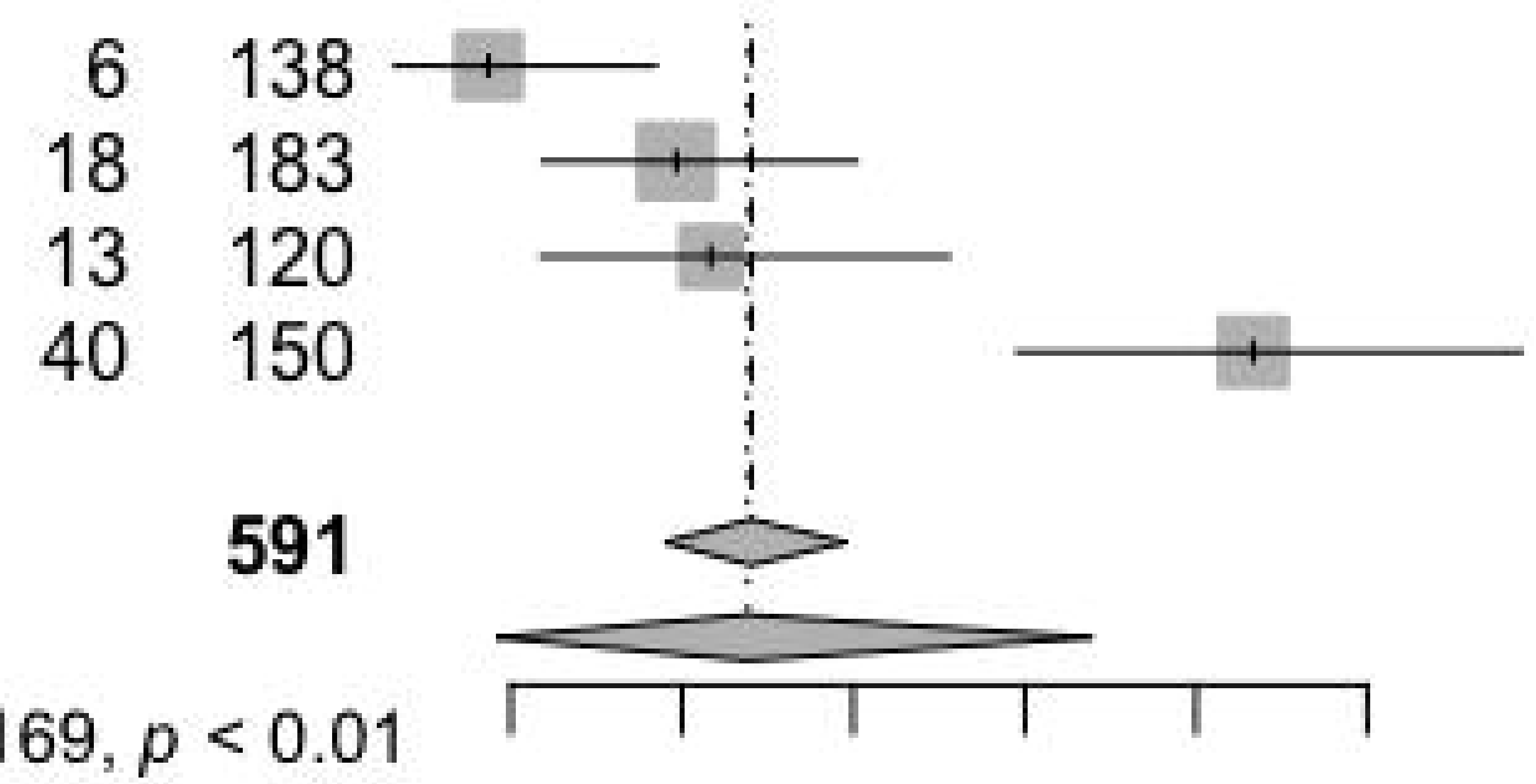

$\begin{array}{llllll}0.05 & 0.1 & 0.15 & 0.2 & 0.25 & 0.3\end{array}$
Weight Weight Proportion $\quad 95 \%-\mathrm{Cl}$ (fixed) (random)

$0.04[0.02 ; 0.09] \quad 23.4 \% \quad 24.9 \%$

$0.10[0.06 ; 0.15] \quad 30.9 \% \quad 25.5 \%$

$0.11[0.06 ; 0.18] \quad 20.3 \% \quad 24.5 \%$

$0.27[0.20 ; 0.34] 25.4 \% \quad 25.1 \%$

$0.12[0.09 ; 0.15] 100.0 \%$

$0.12[0.05 ; 0.22] \quad--100.0 \%$ 
study

With:aren, ri ral.

vara, ob al

Tormagili et is

Jarcbe, et al.

Pork. eits

Trisiosta, $\mathrm{d}$ at

Cariguec. ot al.

Lindi, d a!

Sonrecber, ot 6 .

Arrnsindre. is at [1

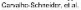

Cerin, ot o

rlaed elfect ncclal

Randam atforte madal

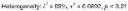

\section{Evosts Totol Ansanla ond hypeanis}

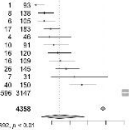

$\begin{array}{lllll}0.1 & 0.2 & 0.3 & 0.1 & 0.5\end{array}$
Weigte Wingt Preportion 15\%,cl [llxcel (raiteri)

nor mao r.co:

2. $7 \mathrm{k}$

4.35

Das [0]3 - [ 11]

3.24

Dos Tha2:[. 12] $2.4 \mathrm{x}$

D.09 0.0: 011 11 $1.2 \%$

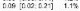

D.11 pas o. 19 2.

Q.13 $0.0 \mathrm{v}: \mathrm{v}$. $2.8 \%$

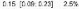

0.18 $0.12 \mathrm{~V} 2 \mathrm{E}$ 3.2\%

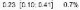

0.27 0.25:0.34 34

ט51 0.A8: C52 12\%

$3.4 \%$

4.45

$1.6 \%$

1.15

$3.3 \%$

$3.4 \%$

4.45

$1.4 \%$

7.95

4.45

$1.5 \%$

0.53 [1.3T;0.40] 110.0\%

0.14 [ $102 ; 020]$

tan. $0 \%$ 


\section{Study}

Tomasini, et al. Kamal, et al.

Events Total

Anxiety

Fixed effect model

Random effects model

Heterogeneity: $I^{2}=73 \%, \tau^{2}=0.0043, p=0.06$

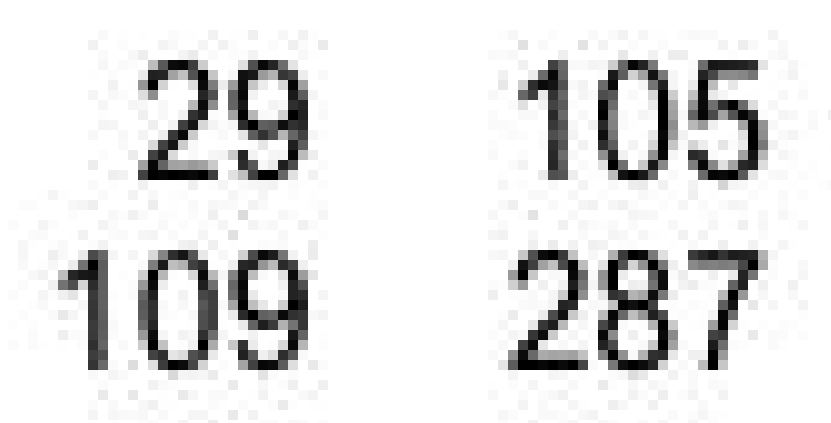

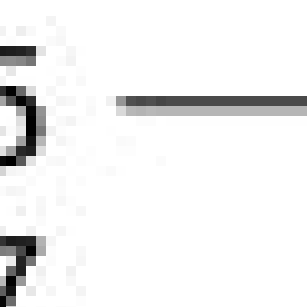

392

$\begin{array}{lllll}0.2 & 0.25 & 0.3 & 0.35 & 0.4\end{array}$
Weight Weight Proportion $\quad 95 \%-\mathrm{Cl}$ (fixed) (random)

$0.28[0.19 ; 0.37] \quad 26.8 \% \quad 43.7 \%$ $0.38[0.32 ; 0.44] \quad 73.2 \% \quad 56.3 \%$

$0.35[0.30 ; 0.40] 100.0 \%$ $0.33[0.24 ; 0.44] \quad--100.0 \%$ 


\section{Study}

Carvalho-Schneider, et al. Jacobs, et al.

Landi, et al.

Carfi, et al.

Taboada, et al.

Kamal, et al.

Fixed effect model

Random effects model

Heterogeneity: $I^{2}=88 \%, \tau^{2}=0.0122, p<0.01 \Gamma$

$\begin{array}{lllllll}0.05 & 0.1 & 0.15 & 0.2 & 0.25 & 0.3 & 0.35\end{array}$
Weight Weight

Proportion $\quad 95 \%-\mathrm{Cl}$ (fixed) (random)

$0.09[0.05 ; 0.14] \quad 15.6 \% \quad 16.8 \%$

$0.16[0.11 ; 0.22] \quad 19.0 \% \quad 17.1 \%$

$0.26[0.18 ; 0.35] \quad 11.3 \% \quad 16.1 \%$

$0.27[0.20 ; 0.35] \quad 14.9 \% \quad 16.7 \%$

$0.29[0.20 ; 0.39] \quad 9.5 \% \quad 15.6 \%$

$0.31[0.26 ; 0.37] 29.8 \% \quad 17.8 \%$

$0.23[0.20 ; 0.25] 100.0 \%$

$0.22[0.15 ; 0.31] \quad--100.0 \%$ 


\section{Study}

Boscolo-Rizzo, et al.

Tomasini, et al.

Carvatho-Schneider, et at: Taboada, et al.

Pellaud, et al.

Fixed effect model

Random effects model

Heterogeneity: $I^{2}=89 \%, \tau^{2}=0.0151, p<0.01$

649

\section{Events Total \\ Asthenia}
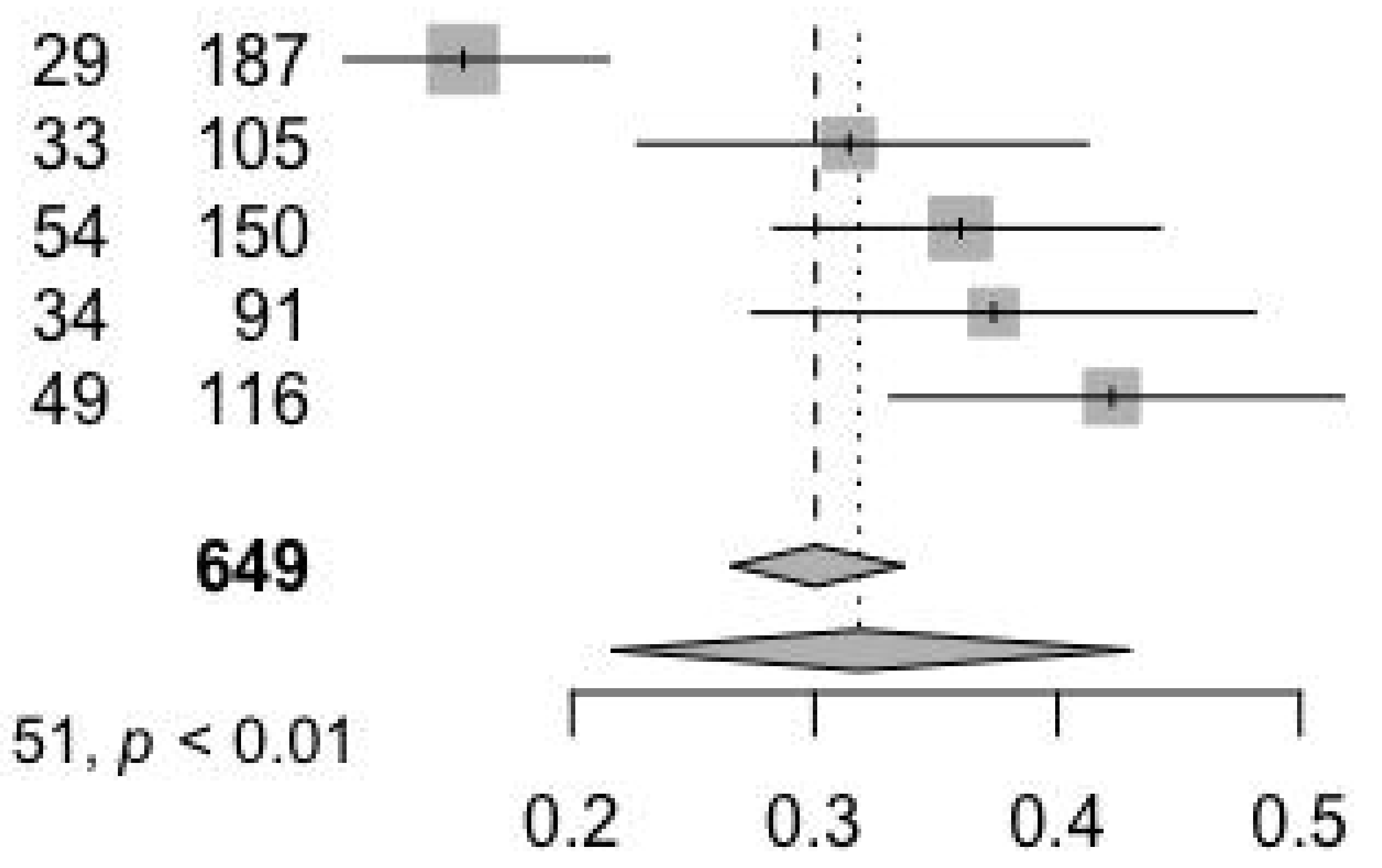

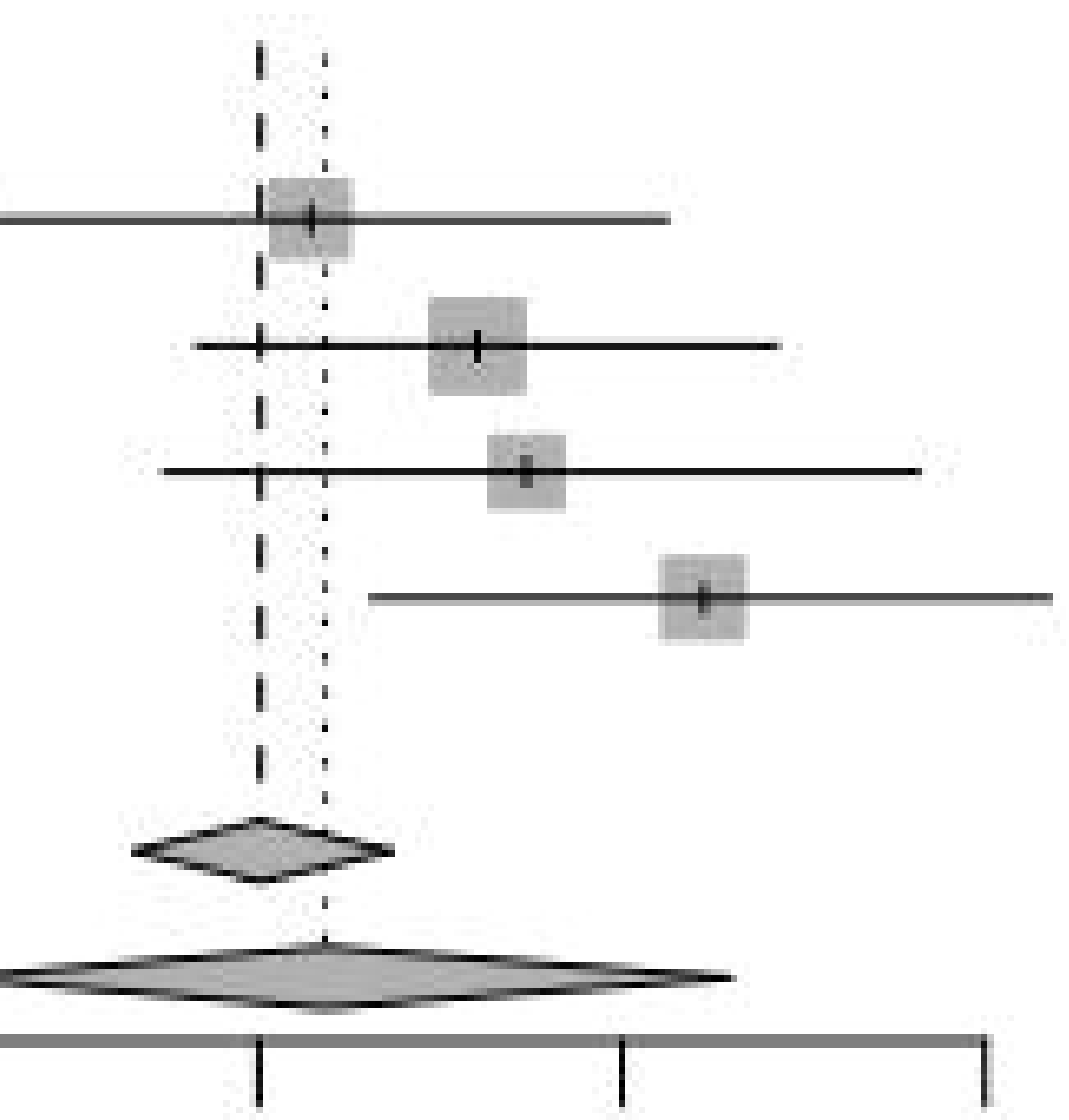

$\begin{array}{llll}0.2 & 0.3 & 0.4 & 0.5\end{array}$

Proportion Weight Weight

$0.16[0.11 ; 0.22] \quad 28.8 \% \quad 20.9 \%$

$0.31[0.23 ; 0.41] \quad 16.2 \% \quad 19.6 \%$

$0.36[0.28 ; 0.44] \quad 23.1 \% \quad 20.4 \%$

$0.37[0.27 ; 0.48] \quad 14.0 \% \quad 19.2 \%$

$0.42[0.33 ; 0.52] \quad 17.9 \% \quad 19.9 \%$

$0.30[0.27 ; 0.34] 100.0 \%$

$0.32[0.22 ; 0.43] \quad--100.0 \%$ 


\begin{tabular}{|c|c|c|c|c|c|c|c|c|}
\hline Study & Events & Total & & Body_aches & Proportion & $95 \%$-Cl & $\begin{array}{r}\text { Weight } \\
\text { (fixed) }\end{array}$ & $\begin{array}{r}\text { Weight } \\
\text { (random) }\end{array}$ \\
\hline Tomasini, et al. & 11 & $105-$ & + & 4 & 0.10 & {$[0.05 ; 0.18]$} & $30.0 \%$ & $32.4 \%$ \\
\hline Halpin, el al & 19 & 100 & & 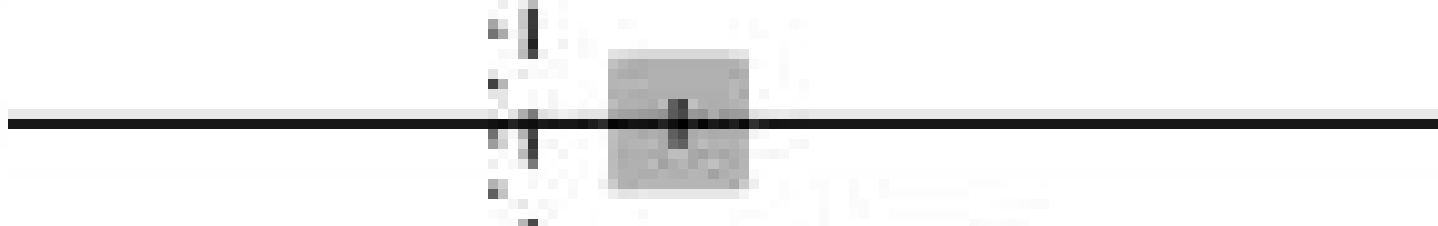 & 0.19 & {$[0.12 ; 0.28]$} & $28.6 \%$ & $31.8 \%$ \\
\hline Sonnweber, et al. & 32 & 145 & & $\begin{array}{c}1 \\
1 \\
1 \\
\vdots \\
1\end{array}$ & 0.22 & {$[0.16 ; 0.30]$} & $41.4 \%$ & $35.8 \%$ \\
\hline $\begin{array}{l}\text { Fixed effect model } \\
\text { Random effects model }\end{array}$ & & 350 & & & $\begin{array}{l}0.17 \\
0.17\end{array}$ & $\begin{array}{l}{[0.14 ; 0.22]} \\
{[0.11 ; 0.25]}\end{array}$ & $\begin{array}{r}100.0 \% \\
-\end{array}$ & $100.0 \%$ \\
\hline \multirow{2}{*}{\multicolumn{3}{|c|}{ Heterogeneity: $I^{2}=68 \%, \tau^{2}=0.0045, p=0.05$}} & . & 7 & & & & \\
\hline & & & 0.1 & $\begin{array}{lll}0.15 & 0.2 & 0.25\end{array}$ & & & & \\
\hline
\end{tabular}




\section{Study}

Boscolo-Rizzo, et al. Landi, et al. Jacobs, et al. Zhao, et al. Park, et al. Caronna, et al. Kamal, et al. Carvalho-Schneider, et al.

Fixed effect model Random effects model

Heterogeneity: $I^{2}=89 \%, \tau^{2}=0.0156, p<0.01$
Events Total Cephalea

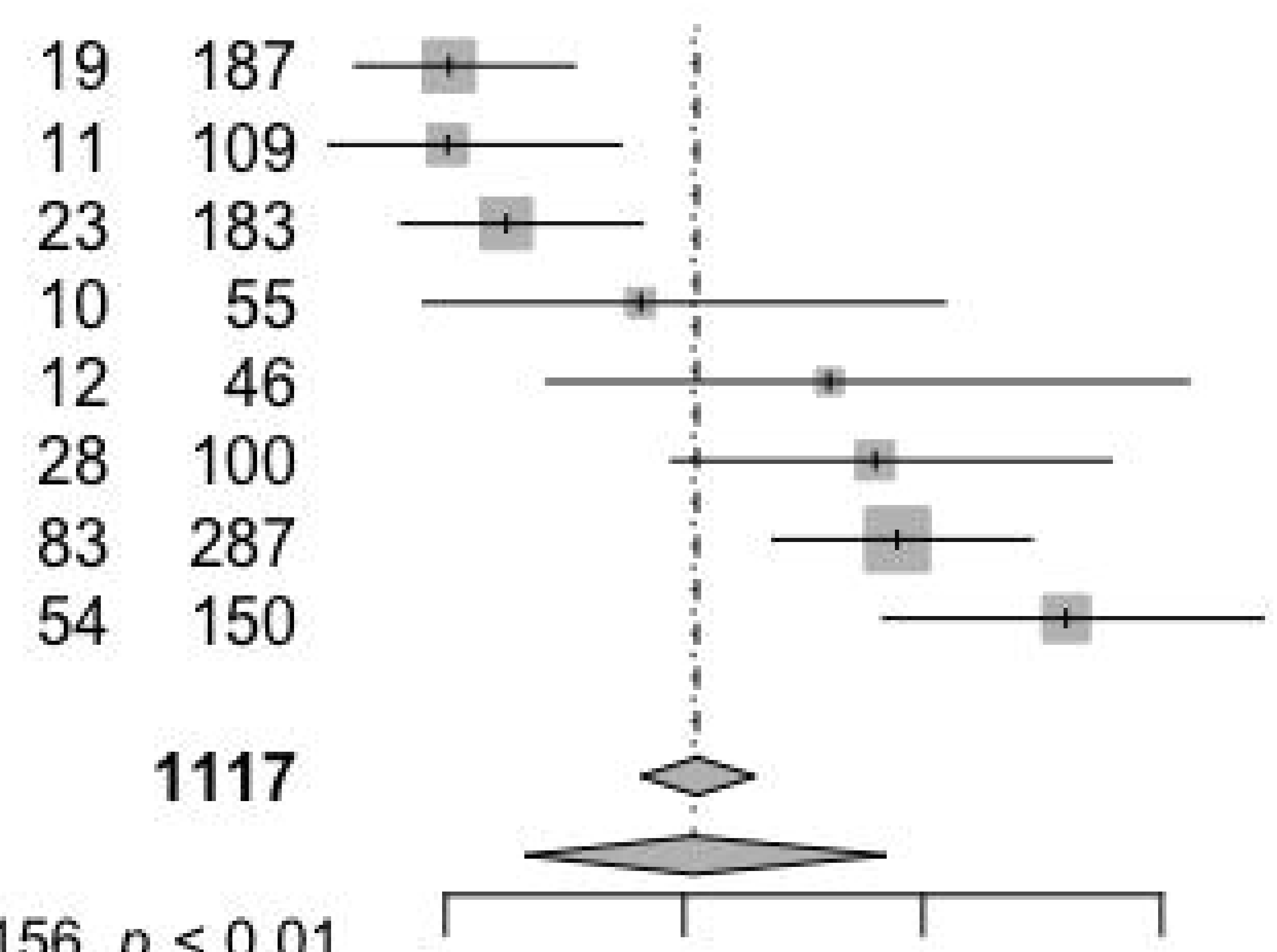

$\begin{array}{llll}0.1 & 0.2 & 0.3 & 0.4\end{array}$
Weight Weight Proportion $\quad 95 \%-\mathrm{Cl}$ (fixed) (random)

$0.10[0.06 ; 0.15] \quad 16.7 \% \quad 13.3 \%$ $0.10[0.05 ; 0.17] \quad 9.8 \% \quad 12.6 \%$ $0.13[0.08 ; 0.18] \quad 16.4 \% \quad 13.2 \%$ $0.18[0.09 ; 0.31] \quad 5.0 \% \quad 11.2 \%$ $0.26[0.14 ; 0.41] \quad 4.1 \% \quad 10.7 \%$ $0.28[0.19 ; 0.38] \quad 9.0 \% \quad 12.4 \%$ $0.29[0.24 ; 0.35] \quad 25.6 \% \quad 13.6 \%$ $0.36[0.28 ; 0.44] \quad 13.4 \% \quad 13.0 \%$

$0.21[0.18 ; 0.23] 100.0 \%$ $0.20[0.13 ; 0.28] \quad-$ -- $\quad 100.0 \%$ 


\section{Study}

Boscolo-Rizzo, et al.

Taboada, et al.

Garrigues, et al.

Huang, et al.

Park, et al.

Carvalho-Schneider, et al.

Carfi, et al.

Kamal, et al.

Fixed effect model

Random effects model

Heterogeneity: $I^{2}=93 \%, \tau^{2}=0.0276, p<0.01$

\section{Events Total}

$\begin{array}{rr}2 & 187 \\ 8 & 91 \\ 13 & 120 \\ 3 & 26 \\ 7 & 46 \\ 27 & 150\end{array}$

$31 \quad 143$

$\begin{array}{ll}83 & 287\end{array}$

1050

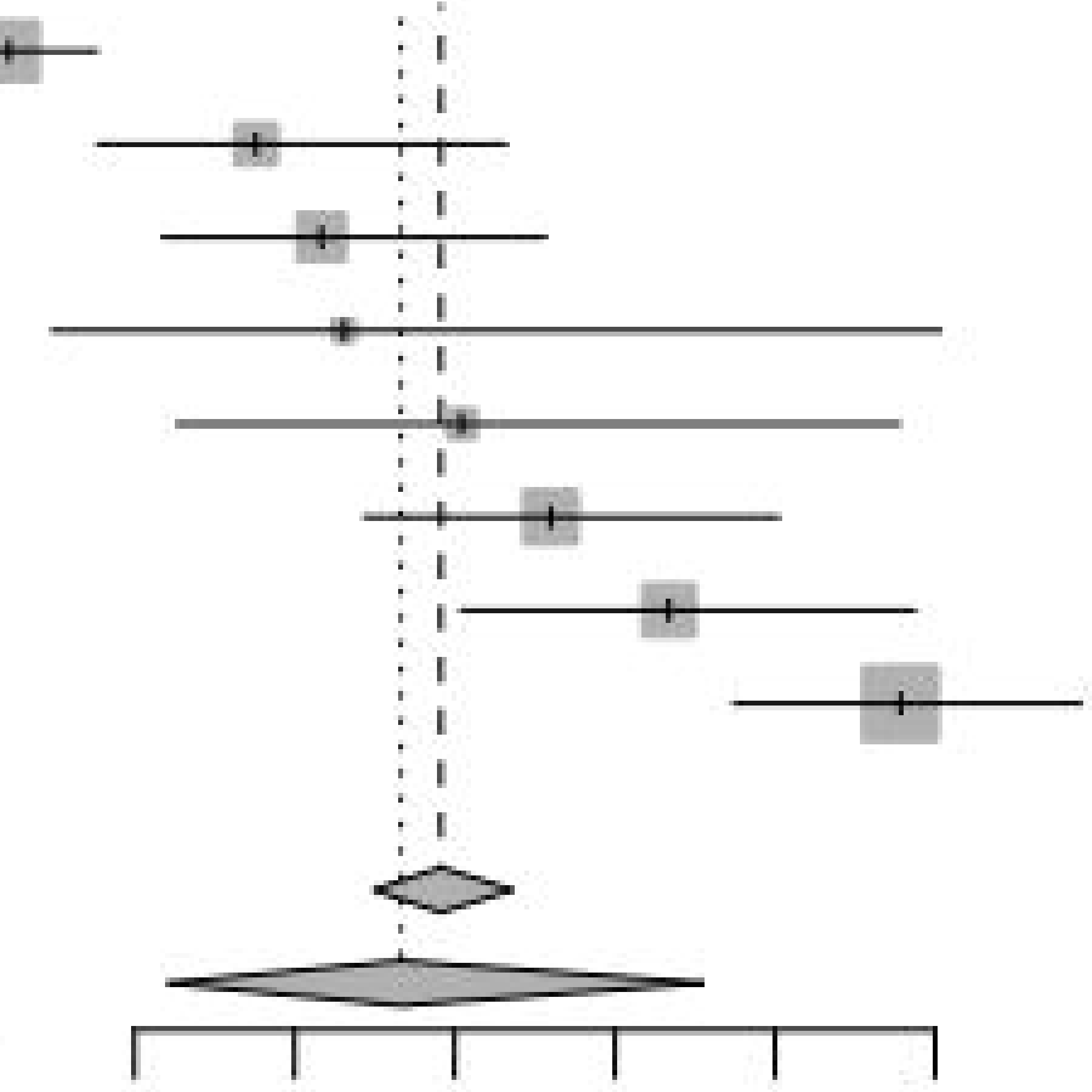

$\begin{array}{llllll}0.05 & 0.1 & 0.15 & 0.2 & 0.25 & 0.3\end{array}$
Weight Weight

Proportion $\quad 95 \%-\mathrm{Cl} \quad$ (fixed) (random)

$0.01[0.00 ; 0.04] \quad 17.8 \% \quad 13.2 \%$

$0.09[0.04 ; 0.17] \quad 8.7 \% \quad 12.6 \%$

$0.11[0.06 ; 0.18] \quad 11.4 \% \quad 12.9 \%$

$0.12[0.02 ; 0.30] \quad 2.5 \% \quad 10.3 \%$

$0.15[0.06 ; 0.29] \quad 4.4 \% \quad 11.6 \%$

$0.18[0.12 ; 0.25] \quad 14.3 \% \quad 13.0 \%$

$0.22[0.15 ; 0.29] \quad 13.6 \% \quad 13.0 \%$

$0.29[0.24 ; 0.35] \quad 27.3 \% \quad 13.4 \%$

$0.15[0.12 ; 0.17] 100.0 \%$

$0.13[0.06 ; 0.23] \quad-$

$-100.0 \%$ 


\section{Study}

Blair, et al.

Taboada, et al.

Landi, et al.

Sonnweber, et al.

Garrigues, et al.

Boscolo-Rizzo, et al. Jacobs, et al.

Park, et al.

Fixed effect model

Random effects model

Heterogeneity: $I^{2}=66 \%, \tau^{2}=0.0041, p<0.01$

$\begin{array}{rr}11 & 84 \\ 13 & 91 \\ 16 & 109 \\ 23 & 145 \\ 20 & 120 \\ 46 & 187 \\ 46 & 183 \\ 17 & 46\end{array}$

965
Events Total Cough
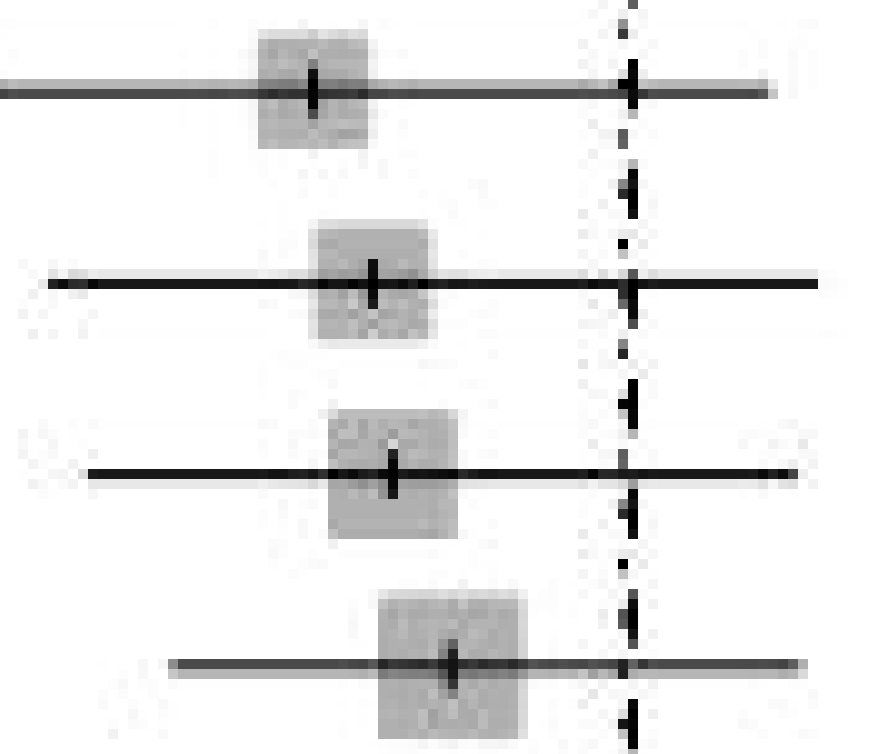

$+1$
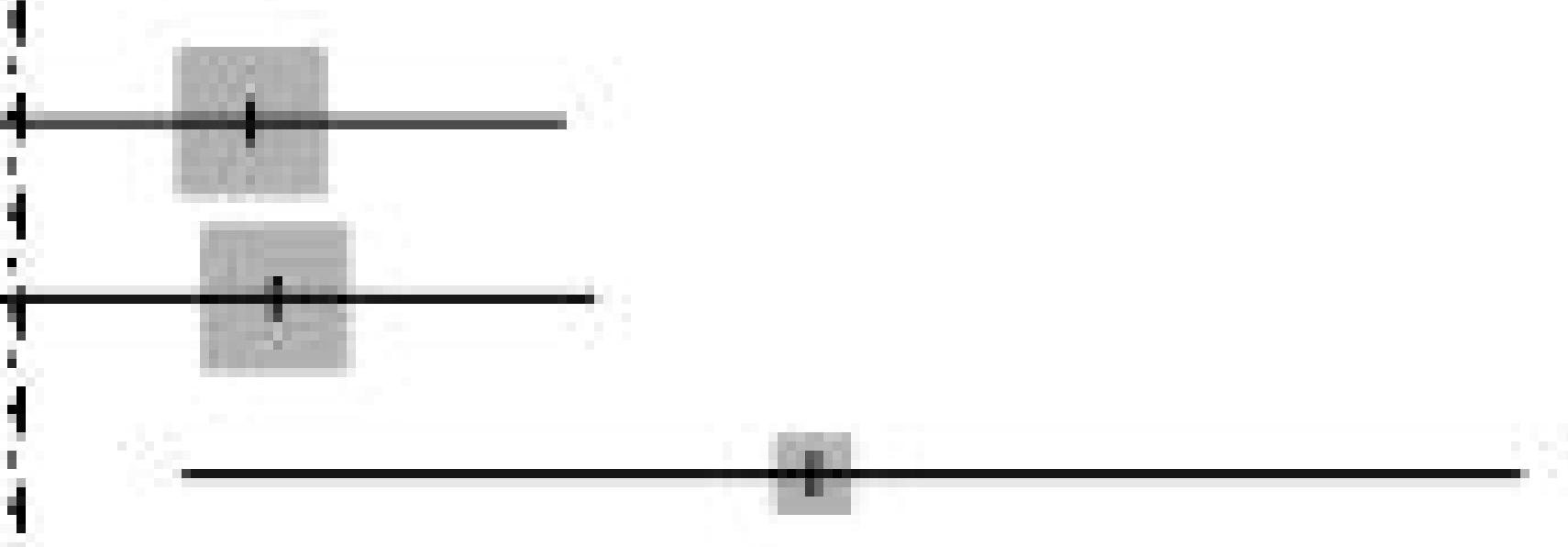

0.1
Weight Weight Proportion $\quad 95 \%-\mathrm{Cl}$ (fixed) (random)

$\begin{array}{rrr}0.13[0.07 ; 0.22] & 8.7 \% & 11.3 \% \\ 0.14[0.08 ; 0.23] & 9.4 \% & 11.7 \% \\ 0.15[0.09 ; 0.23] & 11.3 \% & 12.5 \% \\ 0.16[0.10 ; 0.23] & 15.0 \% & 13.7 \% \\ 0.17[0.10 ; 0.25] & 12.4 \% & 12.9 \% \\ 0.25[0.19 ; 0.31] & 19.3 \% & 14.7 \% \\ 0.25[0.19 ; 0.32] & 18.9 \% & 14.6 \% \\ 0.37[0.23 ; 0.52] & 4.8 \% & 8.5 \%\end{array}$

$0.20[0.17 ; 0.22] 100.0 \%$

$0.19[0.15 ; 0.24]$

- $\quad 100.0 \%$ 
Study

Tomasini, et al. Kamal, et al.

Fixed effect model

Random effects model

Heterogeneity: $I^{2}=94 \%, \tau^{2}=0.0246, p<0.01$

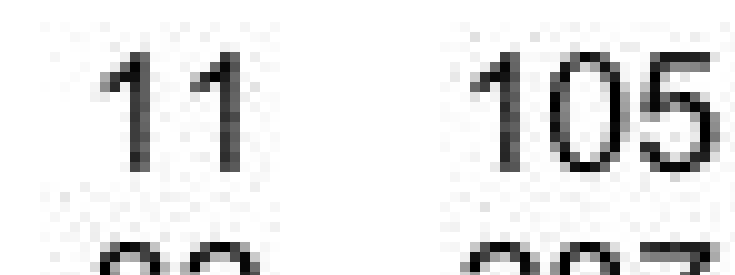

$82 \quad 287$

392
Events Total Depression

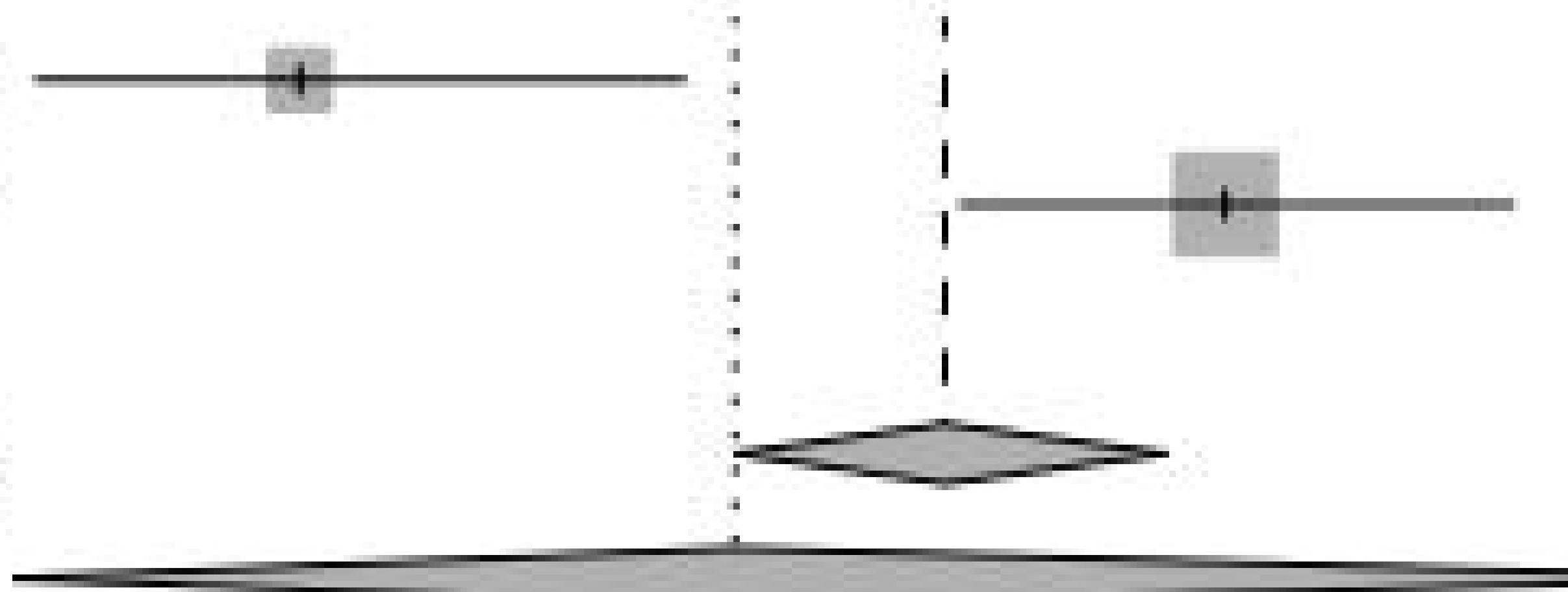

$\begin{array}{lllll}0.1 & 0.15 & 0.2 & 0.25 & 0.3\end{array}$ (fixed) (random)

$0.10[0.05 ; 0.18] \quad 26.8 \% \quad 48.6 \%$ $0.29[0.23 ; 0.34] \quad 73.2 \% \quad 51.4 \%$

$0.23[0.19 ; 0.27] 100.0 \%$ $0.19[0.05 ; 0.39]$

- $100.0 \%$ 


\section{Study}

Jacobs, et al. Landi, et al.

Boscoto-Rizzo, etal.

Sonnweber, et al.

Carvalho-Schneider, et al.

Fixed effect model

Random effects model

Heterogeneity: $I^{2}=82 \%, \tau^{2}=0.0074, p<0.01$

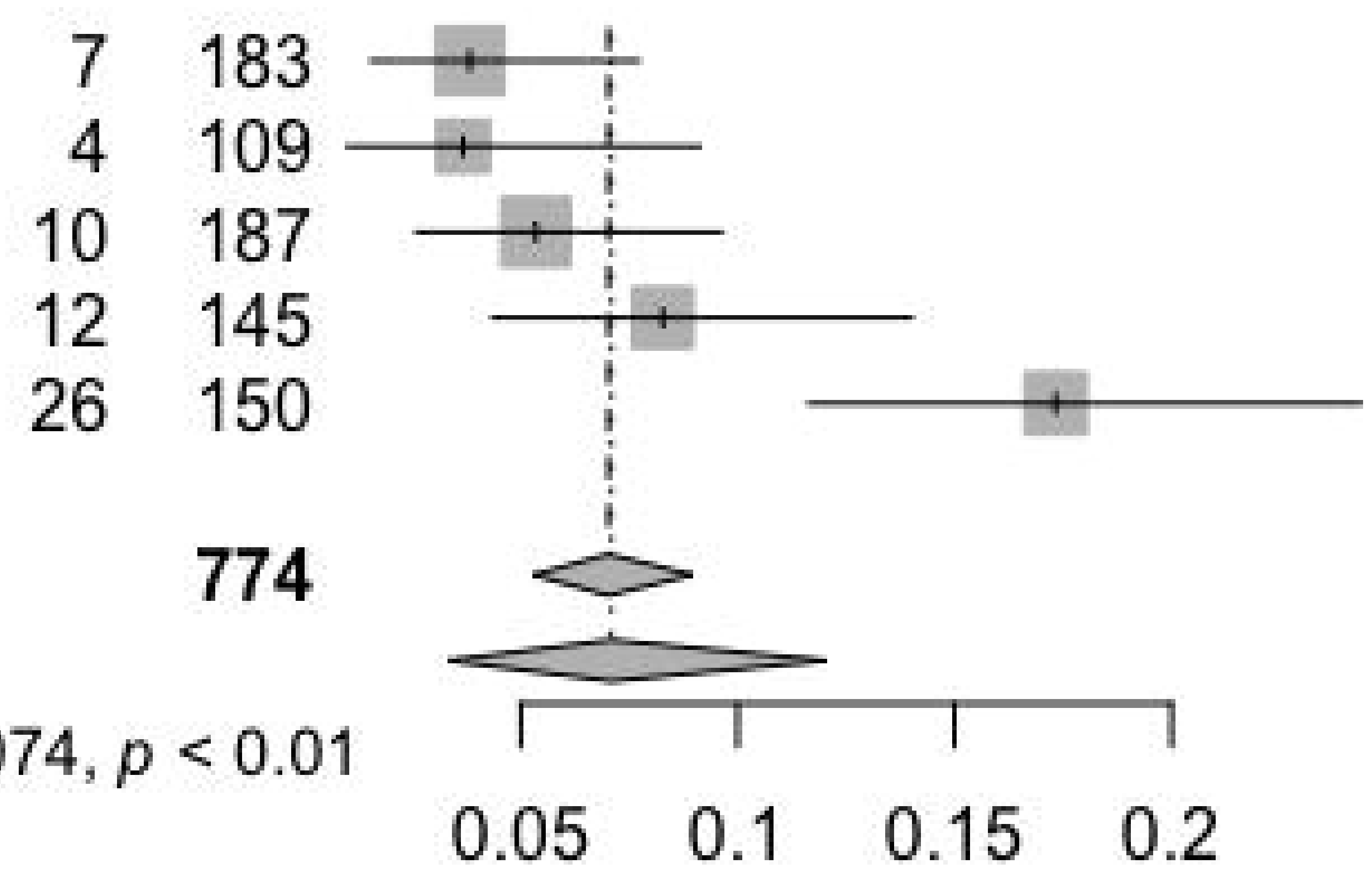

Weight Weight Proportion $\quad 95 \%-\mathrm{Cl}$ (fixed) (random)

$0.04[0.02 ; 0.08] 23.6 \%$ $0.04[0.01 ; 0.09] 14.1 \%$ $0.05[0.03 ; 0.10] \quad 24.1 \%$ $0.08[0.04 ; 0.14] \quad 18.7 \%$ $0.17[0.12 ; 0.24] \quad 19.4 \%$ $20.7 \%$ $18.7 \%$ $20.7 \%$ $19.9 \%$ $20.0 \%$

$0.07[0.05 ; 0.09] 100.0 \%$ $0.07[0.03 ; 0.12]$

$100.0 \%$ 


\section{Study}

Townsend, et al.

Townsend, et al.

Fixed effect model

Random effects model

Heterogeneity: $I^{2}=0 \%, \tau^{2}=0, p=0.47$
Diminished_general

Events Total

$\begin{array}{ll}74 & 128\end{array}$

$95 \quad 153$

281

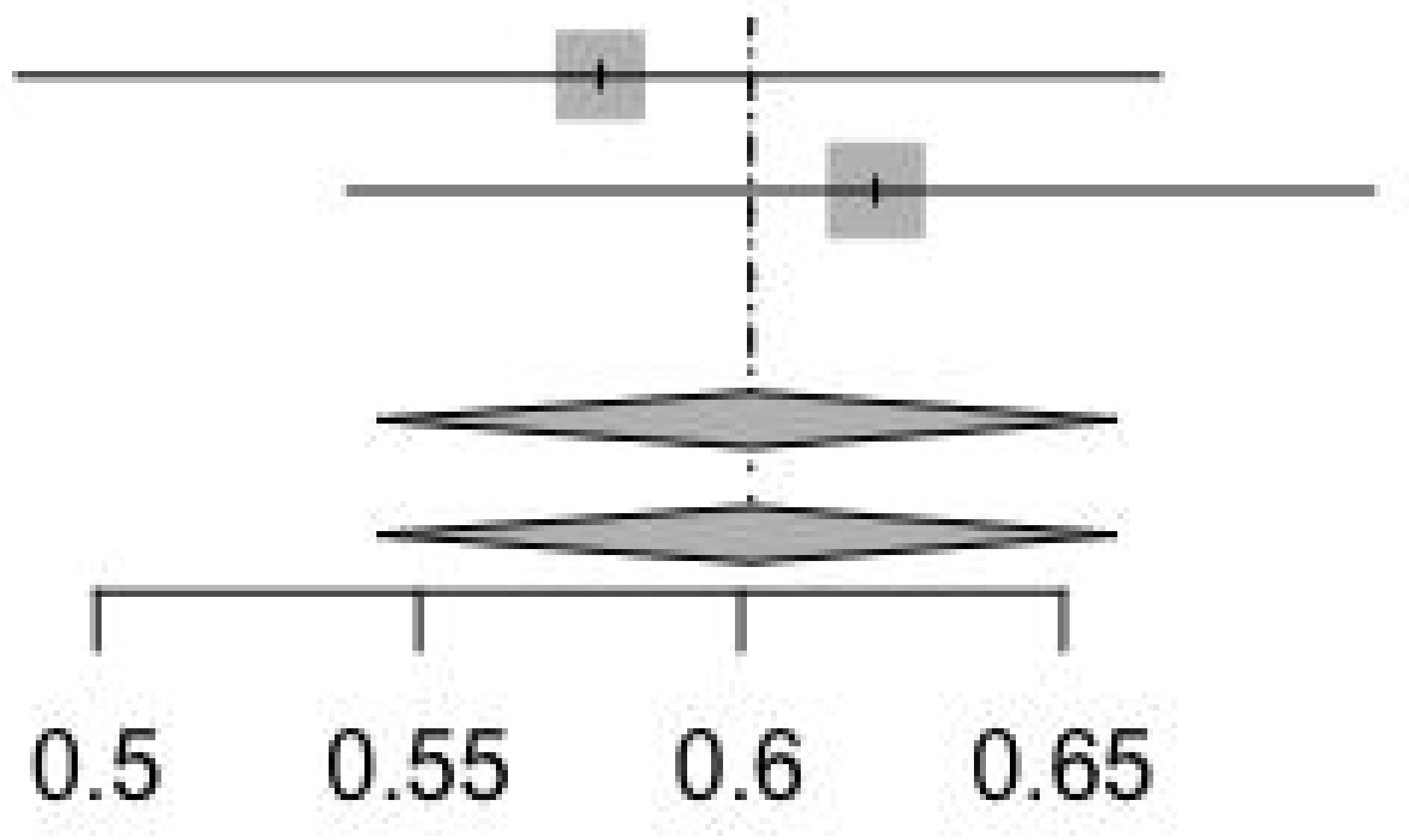

Weight Weight

Proportion $\quad 95 \%-\mathrm{Cl}$ (fixed) (random)

$0.58[0.49 ; 0.66] \quad 45.6 \% \quad 45.6 \%$

$0.62[0.54 ; 0.70] \quad 54.4 \% \quad 54.4 \%$

$0.60[0.54 ; 0.66] 100.0 \%$

$0.60[0.54 ; 0.66]$

$-100.0 \%$ 


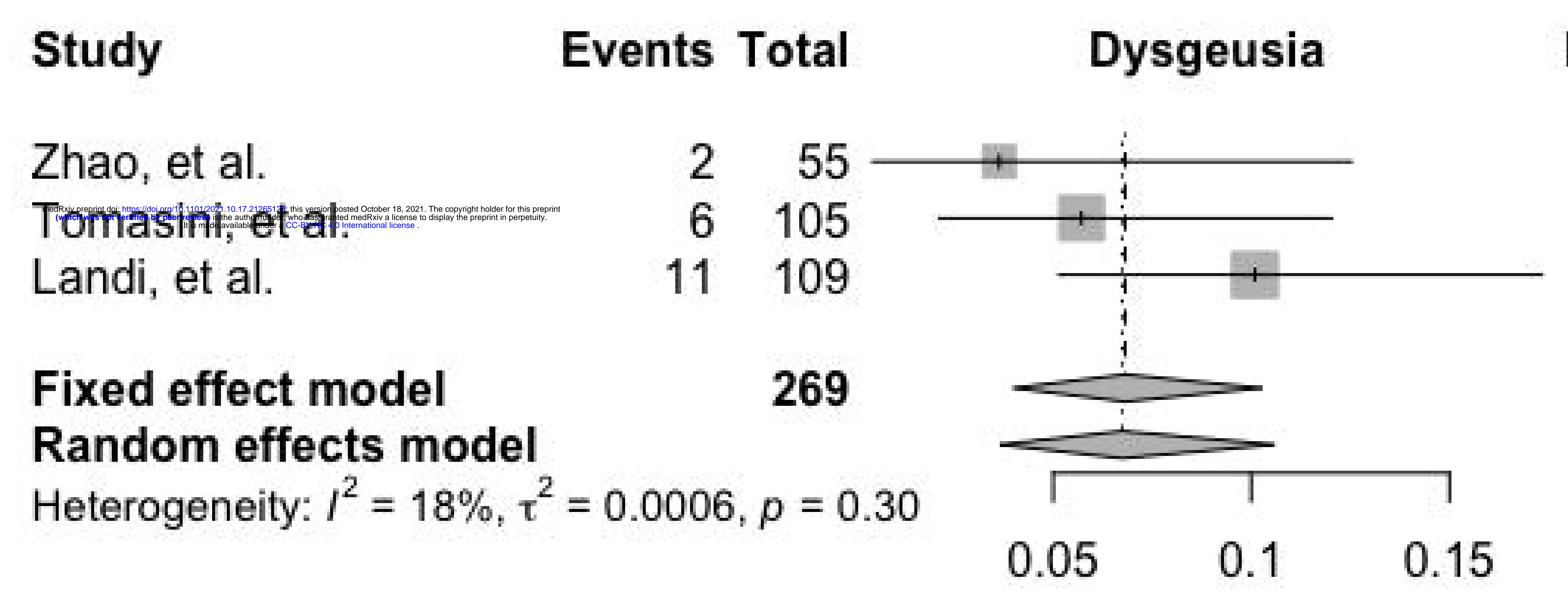

Proportion $\quad 95 \%-\mathrm{Cl} \quad \begin{gathered}\text { Weight } \\ \text { (fixed) }\end{gathered} \begin{array}{r}\text { Weight } \\ \text { (random) }\end{array}$

$0.04[0.00 ; 0.13] \quad 20.5 \% \quad 22.3 \%$

$0.06[0.02 ; 0.12] \quad 39.0 \% \quad 38.3 \%$

$0.10[0.05 ; 0.17] \quad 40.5 \% \quad 39.4 \%$

$0.07[0.04 ; 0.10] 100.0 \%$

$0.07[0.04 ; 0.11] \quad--100.0 \%$ 


\section{Study}

Tomasini, et al.

Carvalho-Schneider, et al. Zhao, et al.

Boscolo-Rizzo, et al.

Kamal, et al. Jacobs, et al.

Sonnweber, etal:

Garrigues, et al.

Carfi, et al. Landi, et al. Halpin, et al.

Raman, et al.

Taboada, et al.

\section{Events Total}

$\begin{array}{ll}7 & 105\end{array}$

$16 \quad 150$

855

$30 \quad 187$

$81 \quad 287$

$\begin{array}{ll}58 & 183\end{array}$

$49 \quad 145$

$\begin{array}{ll}50 & 120\end{array}$

$62 \quad 143$

$\begin{array}{ll}50 & 109\end{array}$

$50 \quad 100$

$36 \quad 58$

$\begin{array}{ll}76 & 91\end{array}$

1733

Fixed effect model

Random effects model

Heterogeneity: $I^{2}=96 \%, \tau^{2}=0.0444, p<0.01$

Weight Weight

Dyspnea

Proportion

$+$

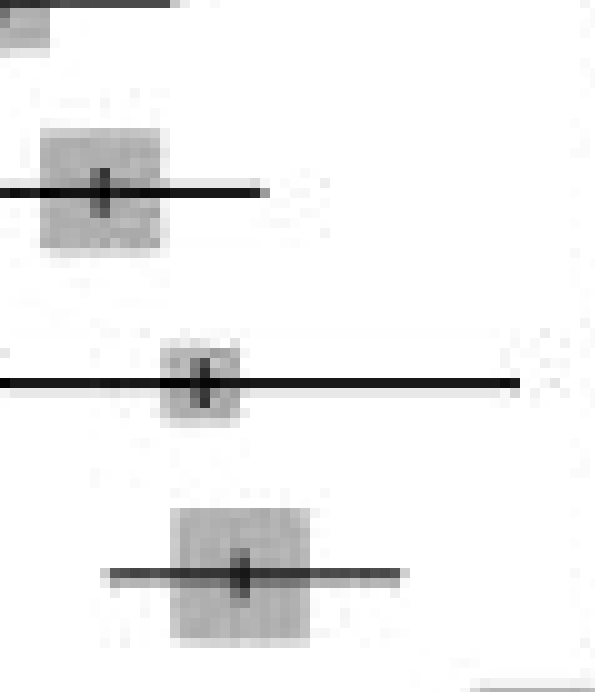

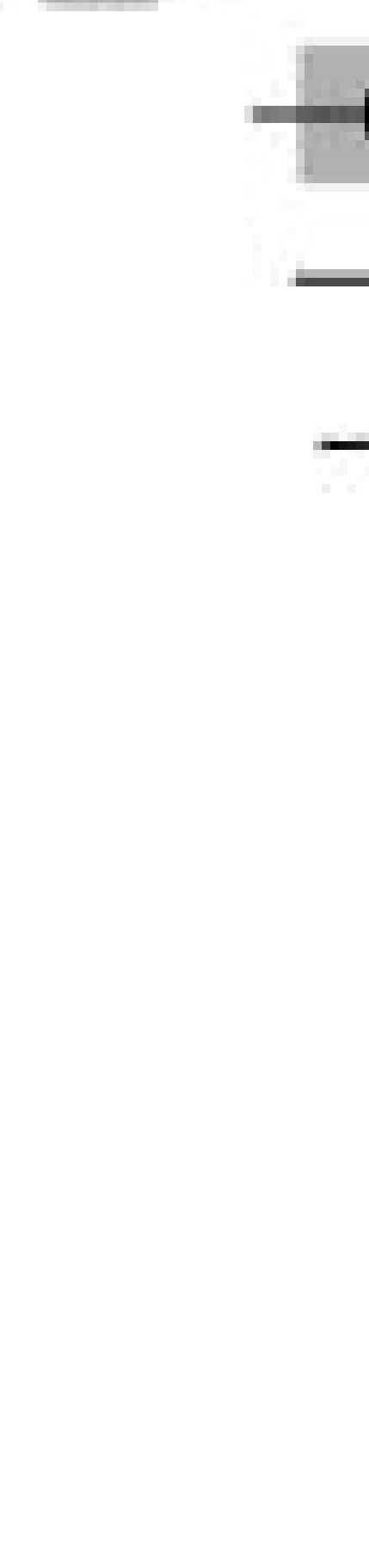
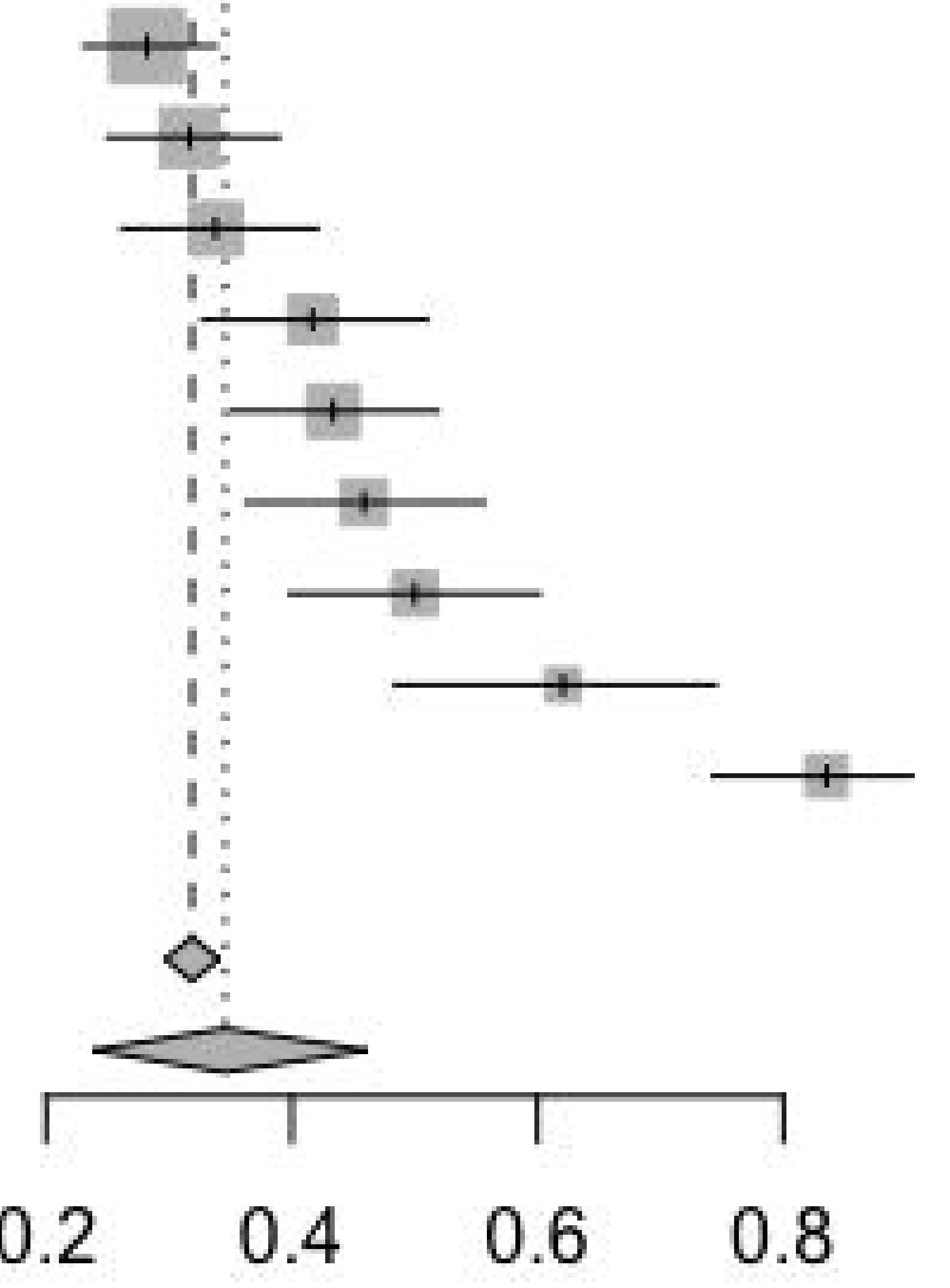

$0.07[0.03 ; 0.13]$

$0.11[0.06 ; 0.17]$

$6.1 \%$

$8.7 \%$

$3.2 \%$

$10.8 \%$

$16.5 \%$

$10.5 \%$

$8.4 \%$

$6.9 \%$

$8.2 \%$

$6.3 \%$

$5.8 \%$

$3.4 \%$

$5.3 \%$

$0.84[0.74 ; 0.90]$

$0.32[0.30 ; 0.34] 100.0 \%$ $0.35[0.24 ; 0.46]$
$7.7 \%$

$7.8 \%$

$7.3 \%$

$7.8 \%$

$7.9 \%$

$7.8 \%$

$7.8 \%$

$7.7 \%$

$7.8 \%$

$7.7 \%$

$7.7 \%$

$7.4 \%$

$7.6 \%$

$100.0 \%$ 


\section{Study}

Zhao, et al.

Park, et al.

Halpin, et al.

Jacobs, et al.

Townsend, et al.

Landi, et al.

Raman, et al.

Townsend, et al.

Carfi, et al.

Garrigues, et al.

Tolba, et al.

Weerahandi, et al.
Events Total

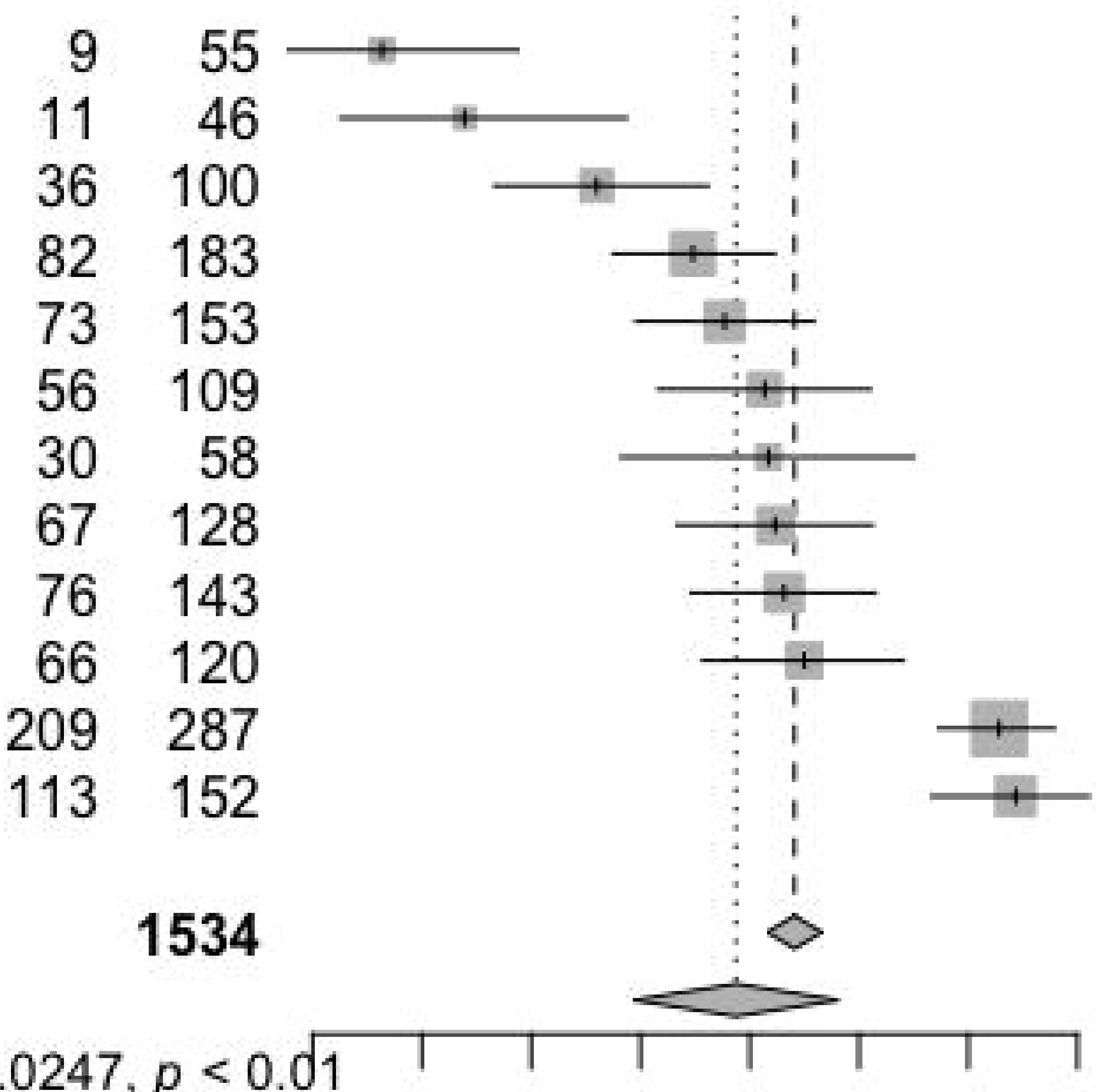

$\begin{array}{llllllll}0.1 & 0.2 & 0.3 & 0.4 & 0.5 & 0.6 & 0.7 & 0.8\end{array}$
Weight Weight

Proportion $\quad 95 \%-\mathrm{Cl}$ (fixed) (random)

\begin{tabular}{lrrr}
$0.16[0.08 ; 0.29]$ & $3.6 \%$ & $7.7 \%$ \\
$0.24[0.13 ; 0.39]$ & $3.0 \%$ & $7.5 \%$ \\
$0.36[0.27 ; 0.46]$ & $6.5 \%$ & $8.3 \%$ \\
$0.45[0.37 ; 0.52]$ & $11.9 \%$ & $8.7 \%$ \\
$0.48[0.40 ; 0.56]$ & $10.0 \%$ & $8.6 \%$ \\
$0.51[0.42 ; 0.61]$ & $7.1 \%$ & $8.4 \%$ \\
$0.52[0.38 ; 0.65]$ & $3.8 \%$ & $7.8 \%$ \\
$0.52[0.43 ; 0.61]$ & $8.3 \%$ & $8.5 \%$ \\
$0.53[0.45 ; 0.62]$ & $9.3 \%$ & $8.6 \%$ \\
$0.55[0.46 ; 0.64]$ & $7.8 \%$ & $8.5 \%$ \\
$0.73[0.67 ; 0.78]$ & $18.7 \%$ & $8.8 \%$ \\
$0.74[0.67 ; 0.81]$ & $9.9 \%$ & $8.6 \%$ \\
\multicolumn{3}{c}{$0.5 \%$} \\
$\mathbf{0 . 5 4}[\mathbf{0 . 5 2} ; \mathbf{0 . 5 7}] \mathbf{1 0 0 . 0 \%}$ & -- \\
$\mathbf{0 . 4 9}[\mathbf{0 . 3 9} \mathbf{0 . 5 8}]$ & -- & $\mathbf{1 0 0 . 0 \%}$
\end{tabular}

Fixed effect model

Random effects model

Heterogeneity: $I^{2}=93 \%, \tau^{2}=0.0247, p<0.01$
Fatigue

$0.49[0.39 ; 0.58]$ 


\section{Study}

Jacobs, et al.

Boscolo-Rizzo, et al.

Park, etal:

Carvalho-Schneider, et al.

Kamal, et al.

Fixed effect model

Random effects model

Heterogeneity: $I^{2}=86 \%, \tau^{2}=0.0091, p<0.01$

\section{Events Total Fever}

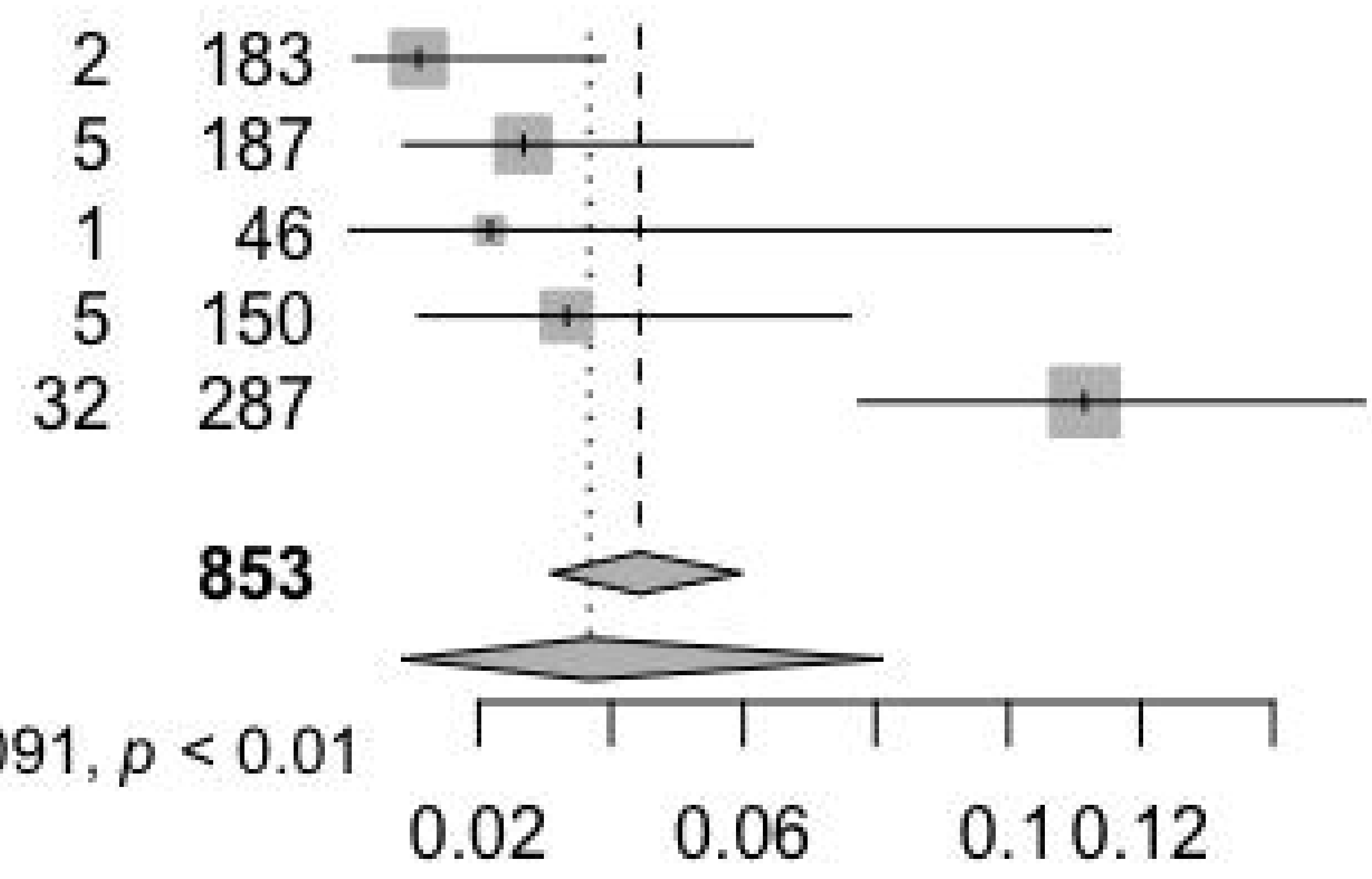

Weight Weight Proportion $\quad 95 \%-\mathrm{Cl}$ (fixed) (random)

$0.01[0.00 ; 0.04] 21.4 \% \quad 21.1 \%$ $0.03[0.01 ; 0.06] \quad 21.9 \% \quad 21.1 \%$ $0.02[0.00 ; 0.12] \quad 5.4 \% \quad 15.2 \%$ $0.03[0.01 ; 0.08] \quad 17.6 \% \quad 20.5 \%$ $0.11[0.08 ; 0.15] \quad 33.6 \% \quad 22.1 \%$

$0.04[0.03 ; 0.06] 100.0 \%$ $0.04[0.01 ; 0.08] \quad--100.0 \%$ 


\section{Study}

Pellaud, et al.

Niklassen, et al

Boscolo-Rizzo, et al.

Konstantinidis, et al.

Fixed effect model

Random effects model

Heterogeneity: $I^{2}=92 \%, \tau^{2}=0.0292, p<0.01$

Events Total Hyposmia and dysgeusia Proportion

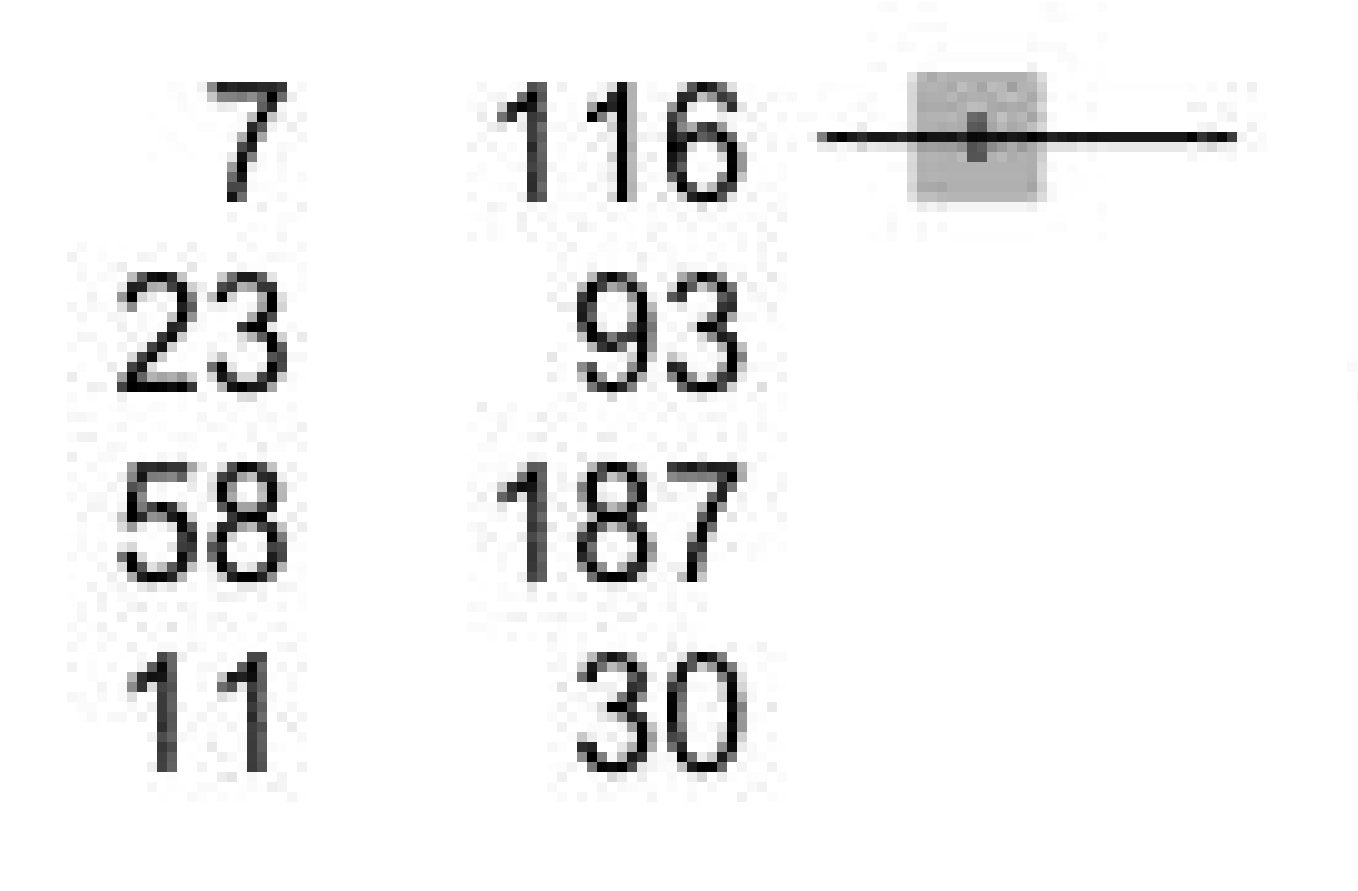

426

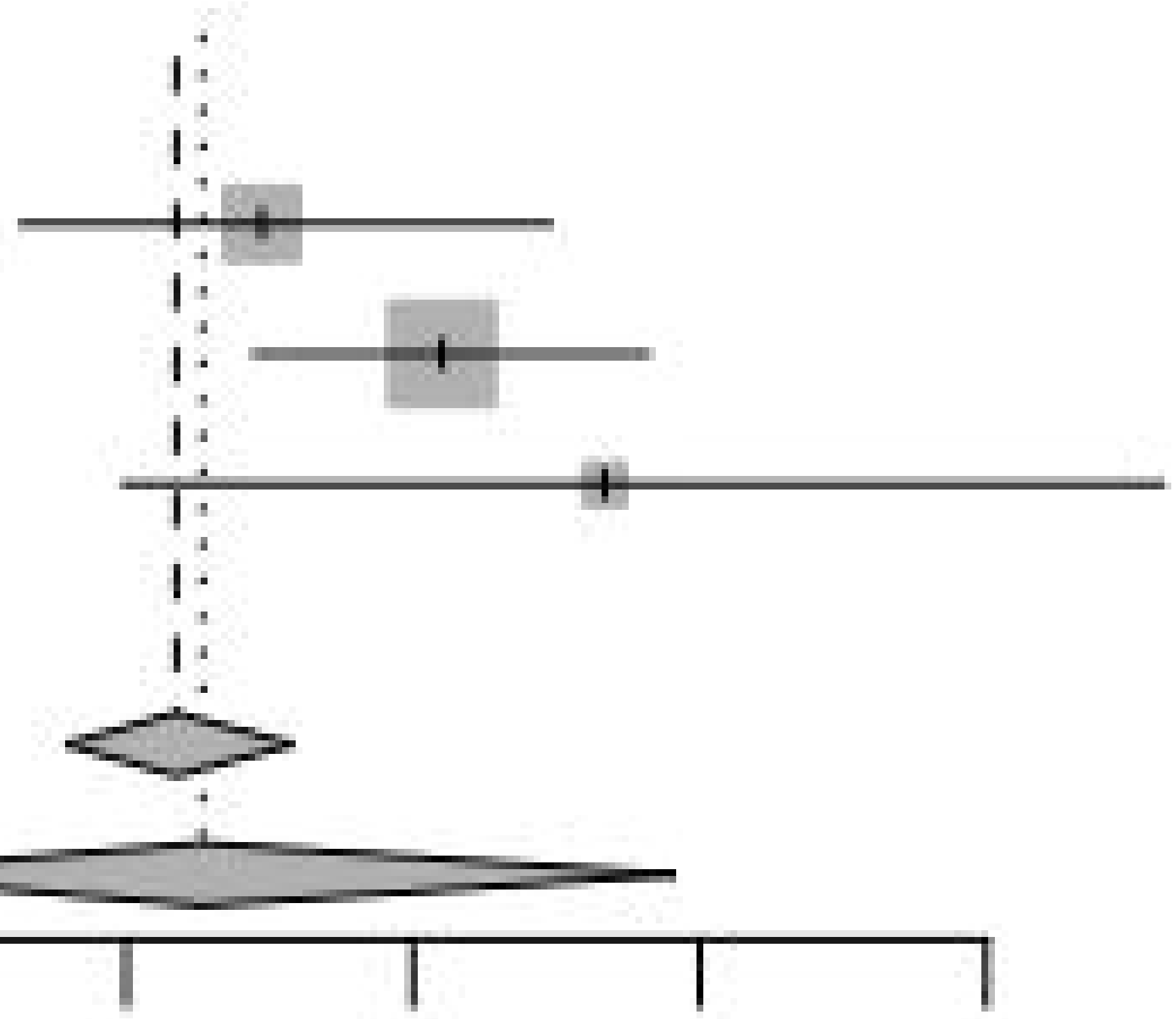

$\begin{array}{lllll}0.1 & 0.2 & 0.3 & 0.4 & 0.5\end{array}$
Weight Weight $95 \%-\mathrm{Cl}$ (fixed) (random)

$0.06[0.02 ; 0.12] \quad 27.2 \% \quad 26.0 \%$

$0.25[0.16 ; 0.35] \quad 21.8 \% \quad 25.6 \%$

$0.31[0.24 ; 0.38] \quad 43.8 \% \quad 26.7 \%$

$0.37[0.20 ; 0.56] \quad 7.1 \% \quad 21.8 \%$

$0.22[0.18 ; 0.26] 100.0 \%$

$0.23[0.10 ; 0.39]$

$-100.0 \%$ 


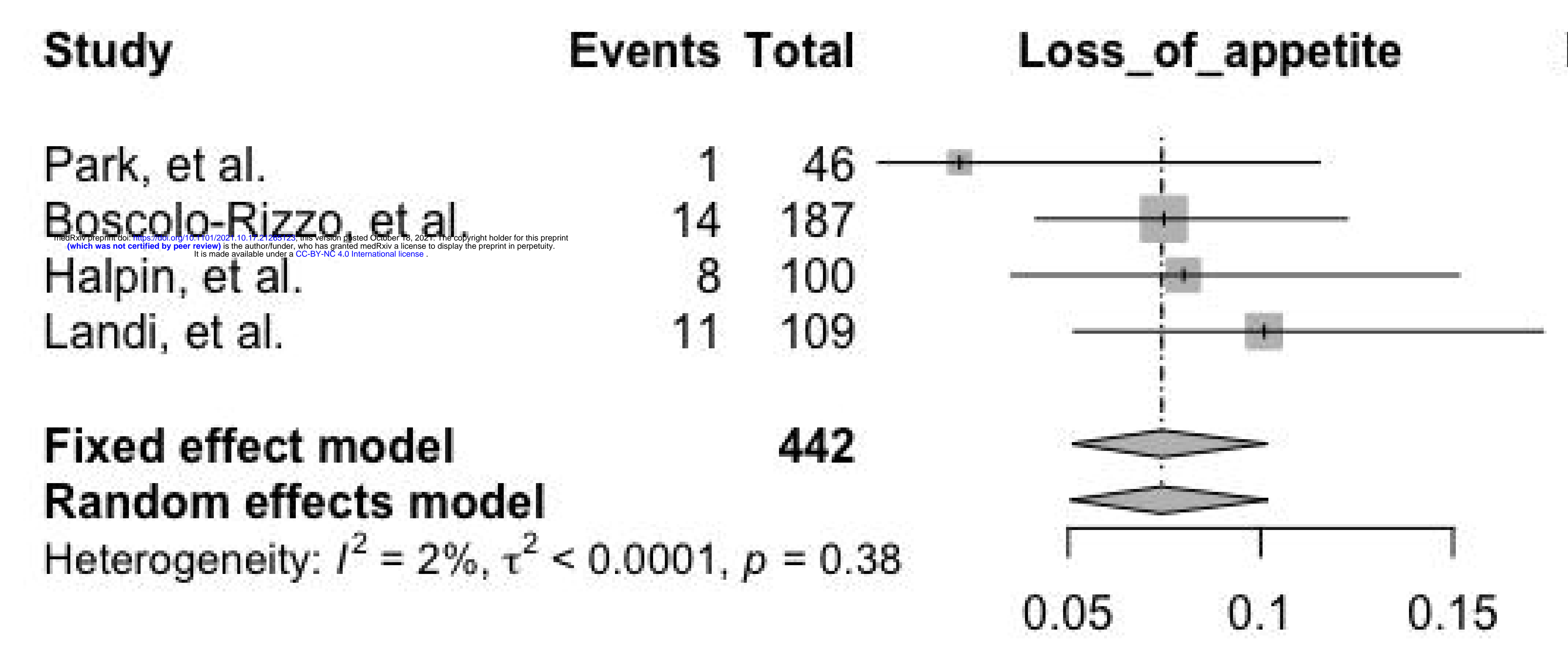

\begin{abstract}
Weight Weight Proportion $\quad 95 \%-\mathrm{Cl}$ (fixed) (random)
\end{abstract}

$\begin{array}{lrrr}0.02[0.00 ; 0.12] & 10.5 \% & 10.7 \% \\ 0.07[0.04 ; 0.12] & 42.2 \% & 41.8 \% \\ 0.08[0.04 ; 0.15] & 22.6 \% & 22.8 \% \\ 0.10[0.05 ; 0.17] & 24.7 \% & 24.8 \% \\ & & \\ \mathbf{0 . 0 7}[\mathbf{0 . 0 5} ; \mathbf{0 . 1 0}] & \mathbf{1 0 0 . 0 \%} & - \\ \mathbf{0 . 0 7}[\mathbf{0 . 0 5} ; \mathbf{0 . 1 0}] & -- & \mathbf{1 0 0 . 0 \%}\end{array}$




\section{Study}

Park, et al.

Jacobs, et al.

Carvalho-Schneider, et al.

Taboada, et al.

Fixed effect model

Random effects model

Heterogeneity: $I^{2}=84 \%, \tau^{2}=0.0116, p<0.01$

\section{Events Total Myalgias}

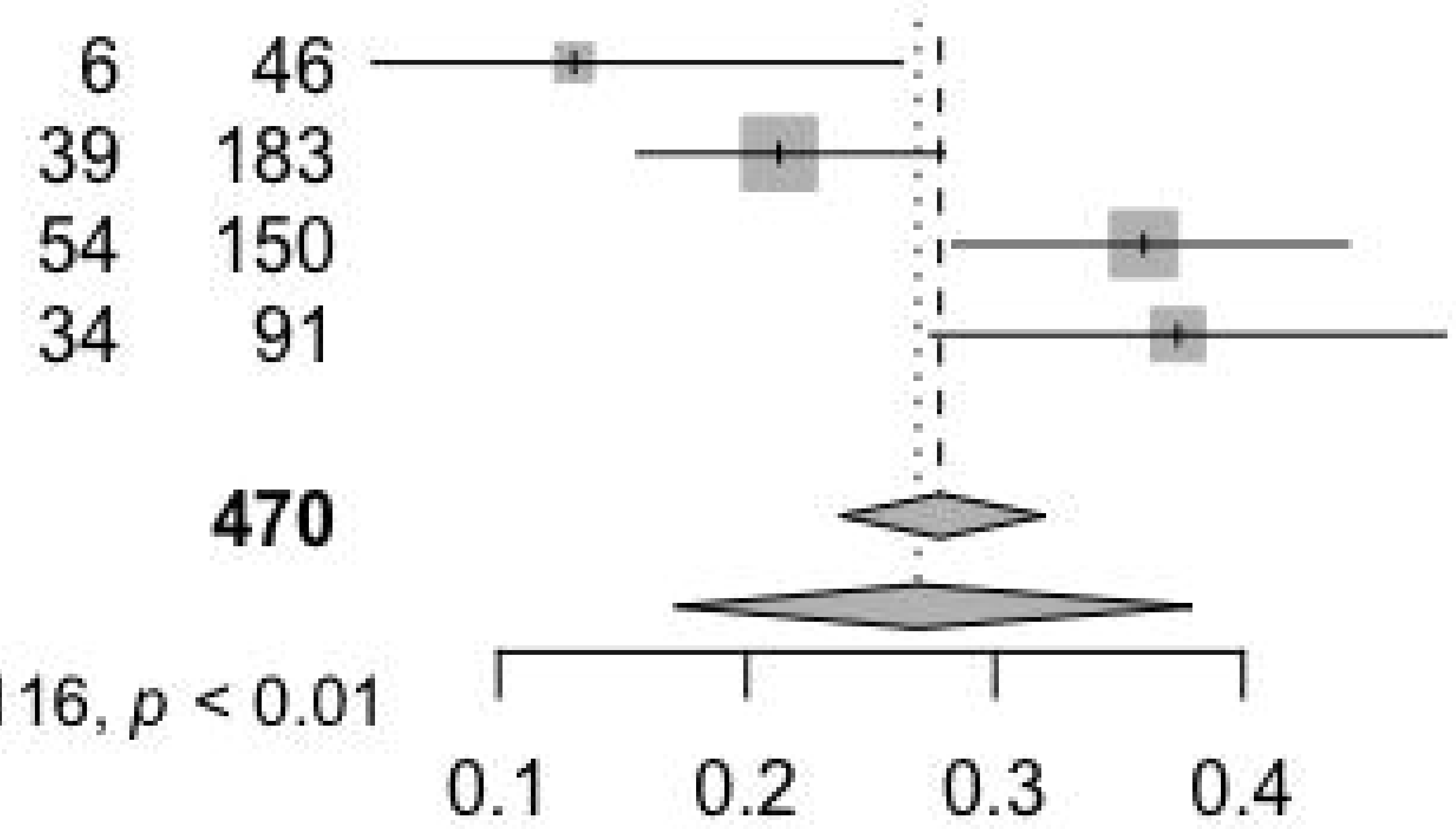

Weight Weight Proportion $\quad 95 \%-\mathrm{Cl}$ (fixed) (random)

$0.13[0.05 ; 0.26] \quad 9.9 \% \quad 20.9 \%$

$0.21[0.16 ; 0.28] \quad 38.9 \% \quad 27.4 \%$

$0.36[0.28 ; 0.44] \quad 31.9 \% \quad 26.8 \%$

$0.37[0.27 ; 0.48] \quad 19.4 \% \quad 24.8 \%$

$0.28[0.24 ; 0.32] 100.0 \%$

$0.27[0.17 ; 0.38] \quad--100.0 \%$ 


\section{Study}

Vaira, et al.

Barón-Sánchez, et al. [ ]

Konstantinidis, et al.

Sonnweber, et al.

Myall, et al.

Gerkin, et al.

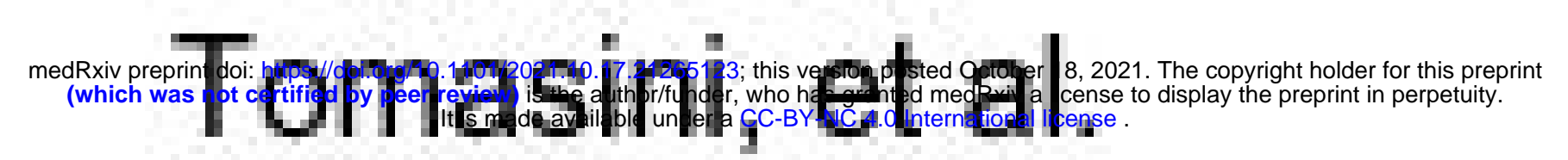

Caronna, et al.

Gambini, et al.

Pellaud, et al.

Carvalho-Schneider, et al.

Weerahandi, et al.

Taboada, et al.

Carfi, et al.

Tolba, et al.

Fixed effect model

Random effects model

Events Total

PACS prevalence

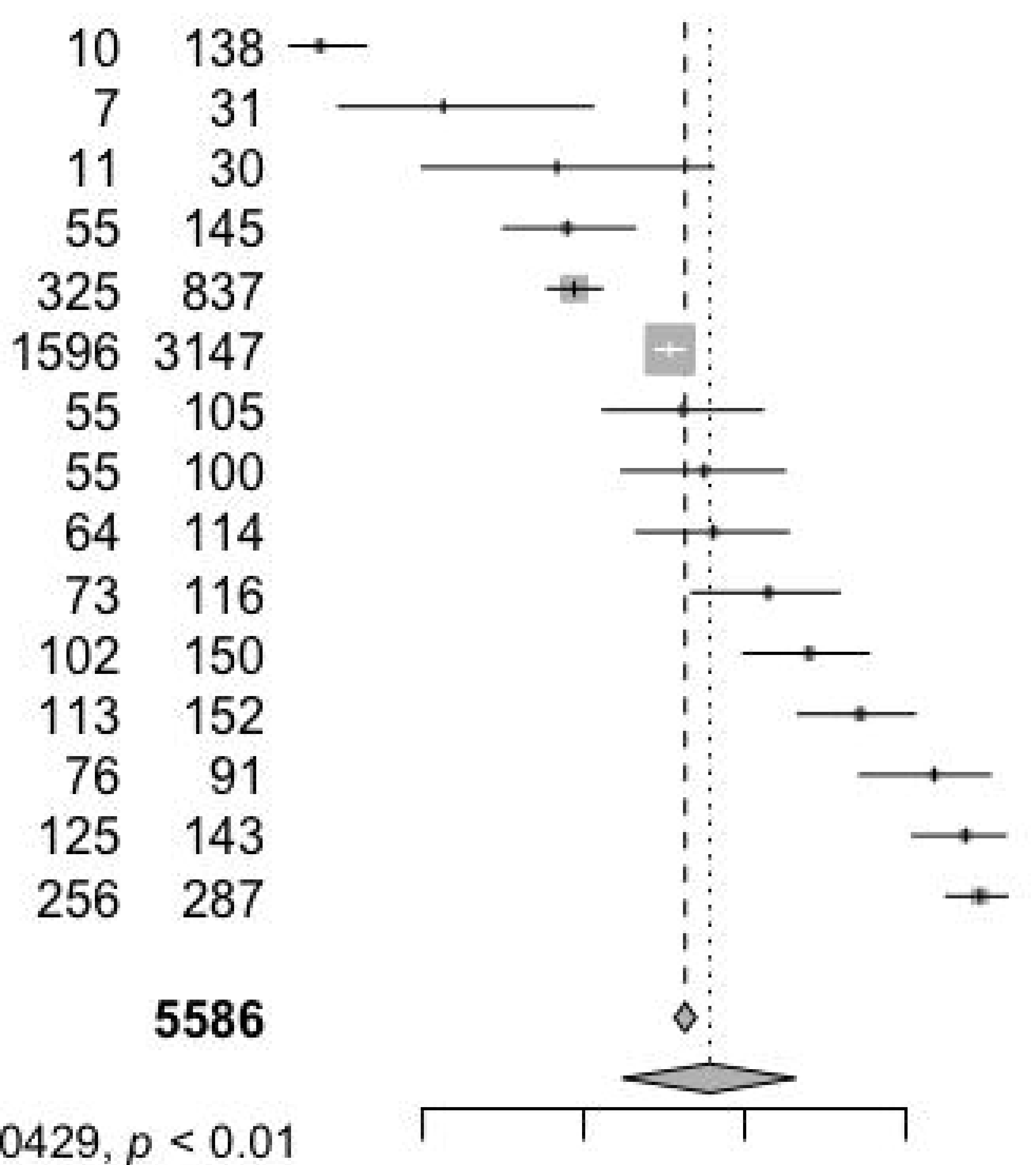

0.2

$\begin{array}{lll}0.4 & 0.6 & 0.8\end{array}$

Proportion

$0.07[0.04 ; 0.13]$

$0.23[0.10 ; 0.41]$

$0.37[0.20 ; 0.56]$

$0.38[0.30 ; 0.46]$

$0.39[0.36 ; 0.42]$

$0.51[0.49 ; 0.52]$

$0.52[0.42 ; 0.62]$

$0.55[0.45 ; 0.65]$

$0.56[0.47 ; 0.65]$

$0.63[0.53 ; 0.72]$

$0.68[0.60 ; 0.75]$

$0.74[0.67 ; 0.81]$

$0.84[0.74 ; 0.90]$

$0.87[0.81 ; 0.92]$

$0.89[0.85 ; 0.93]$

$0.53[0.51 ; 0.54] 100.0 \%$

$0.56[0.45 ; 0.66$ ]
Weight Weight

(fixed) (random)

$2.5 \%$

$0.6 \%$

$0.5 \%$

$2.6 \%$

$15.0 \%$

$56.3 \%$

$1.9 \%$

$1.8 \%$

$2.0 \%$

$2.1 \%$

$2.7 \%$

$2.7 \%$

$1.6 \%$

$2.6 \%$

$5.1 \%$

$6.8 \%$

$5.9 \%$

$5.9 \%$

$6.8 \%$ $7.0 \%$ $7.0 \%$

$6.7 \%$

$6.7 \%$

$6.7 \%$

$6.7 \%$

$6.8 \%$

$6.8 \%$

$6.6 \%$

$6.8 \%$

$6.9 \%$

Heterogeneity: $I^{2}=98 \%, \tau^{2}=0.0429, p<0.01$ 


\section{Study}

Sonnweber, et al _........... Huang, et al.

Fixed effect model

Random effects model

Heterogeneity: $I^{2}=95 \%, \tau^{2}=0.0979, p<0.01$
Events Total Pericardial_efussion

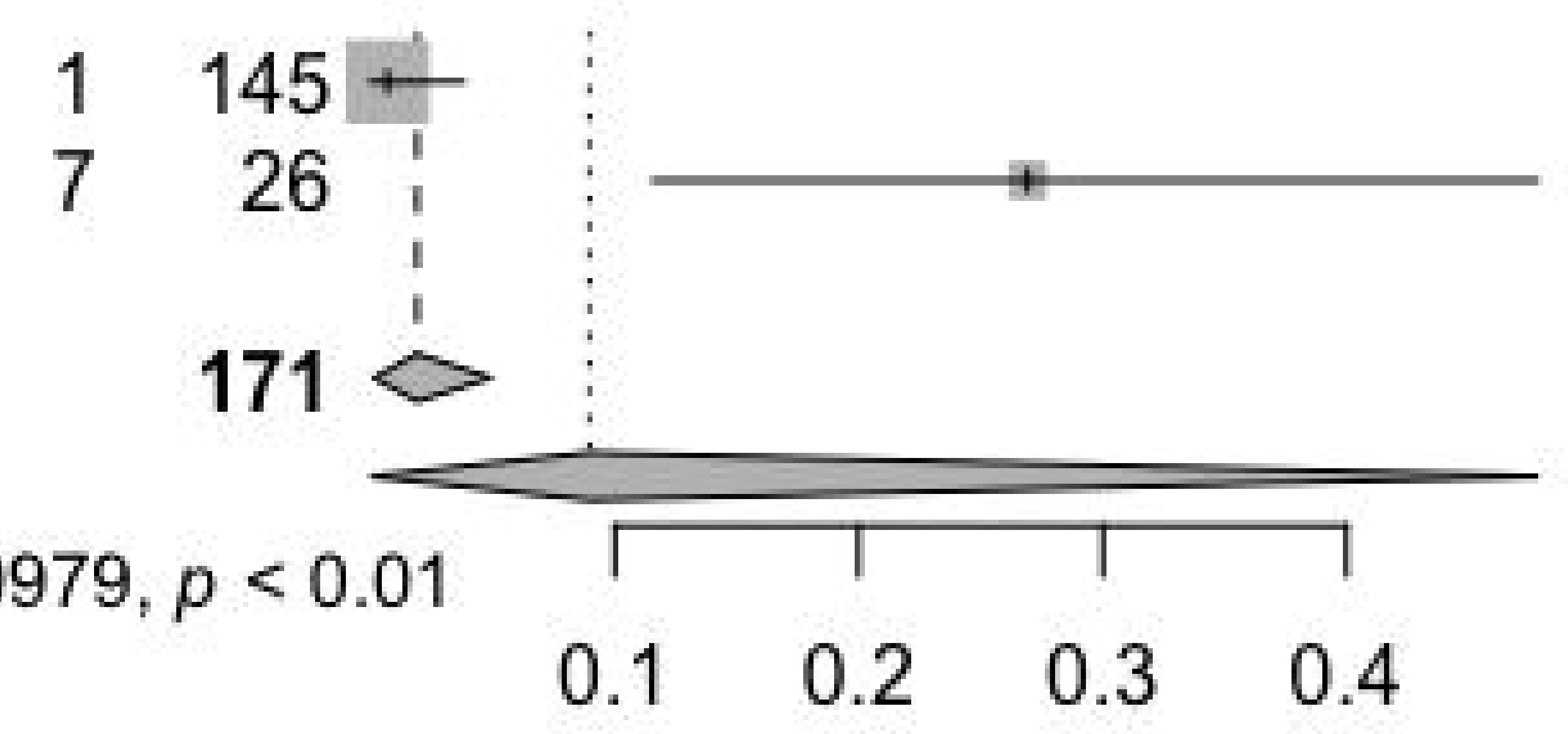

Proportion $\quad 95 \%-\mathrm{Cl}$ (fixed) (random)

$0.01[0.00 ; 0.04] \quad 84.6 \% \quad 51.9 \%$

$0.27[0.12 ; 0.48] \quad 15.4 \% \quad 48.1 \%$

$0.02[0.00 ; 0.05] 100.0 \%$ $0.09[0.00 ; 0.48]$

$-100.0 \%$ 


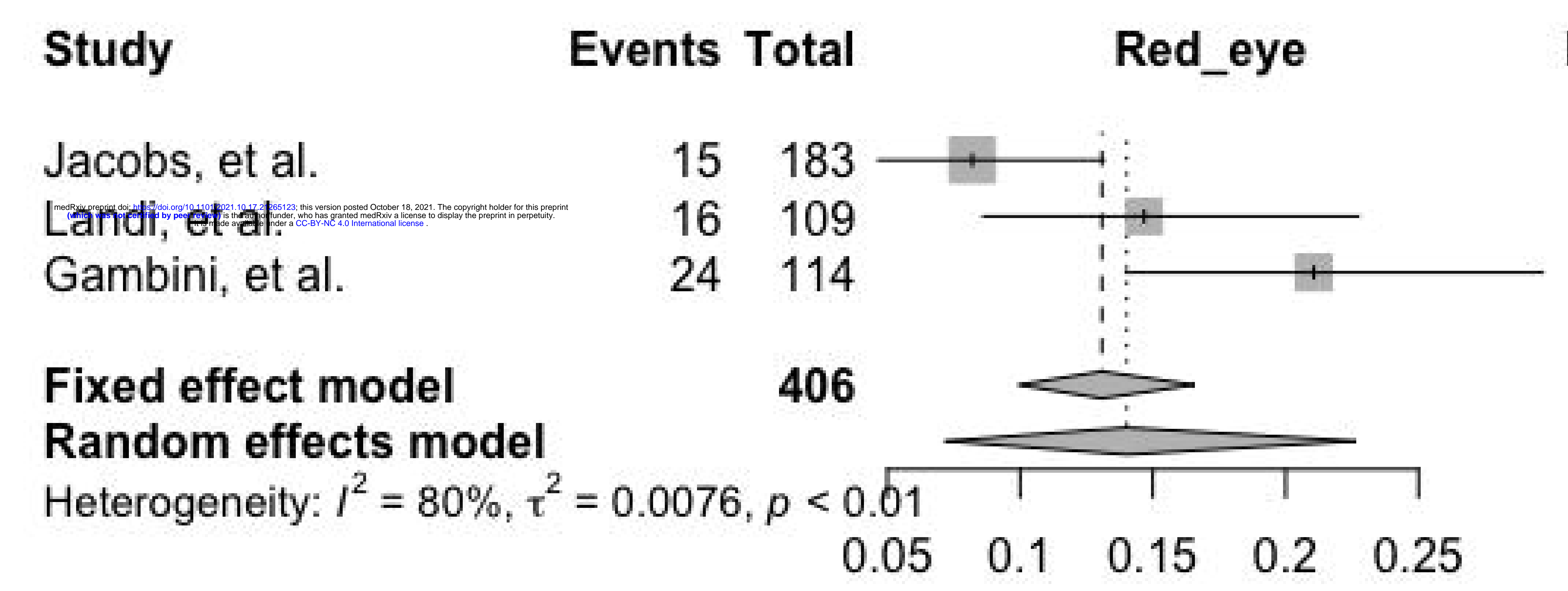

Proportion $\quad 95 \%-\mathrm{Cl} \quad \begin{gathered}\text { Weight } \\ \text { (fixed) }\end{gathered} \begin{array}{r}\text { Weight } \\ \text { (random) }\end{array}$

$0.08[0.05 ; 0.13] \quad 45.0 \% \quad 35.4 \%$

$0.15[0.09 ; 0.23] \quad 26.9 \% \quad 32.1 \%$

$0.21[0.14 ; 0.30] \quad 28.1 \% \quad 32.5 \%$

$0.13[0.10 ; 0.17] 100.0 \%$

$0.14[0.07 ; 0.23] \quad--100.0 \%$ 


\section{Study}

Konstantinidis, et al. Boscolo-Rizzo, et al. Landi, et al.

Park, et al.

Fixed effect model Random effects model

Heterogeneity: $I^{2}=89 \%, \tau^{2}=0.0255, p<0.01$

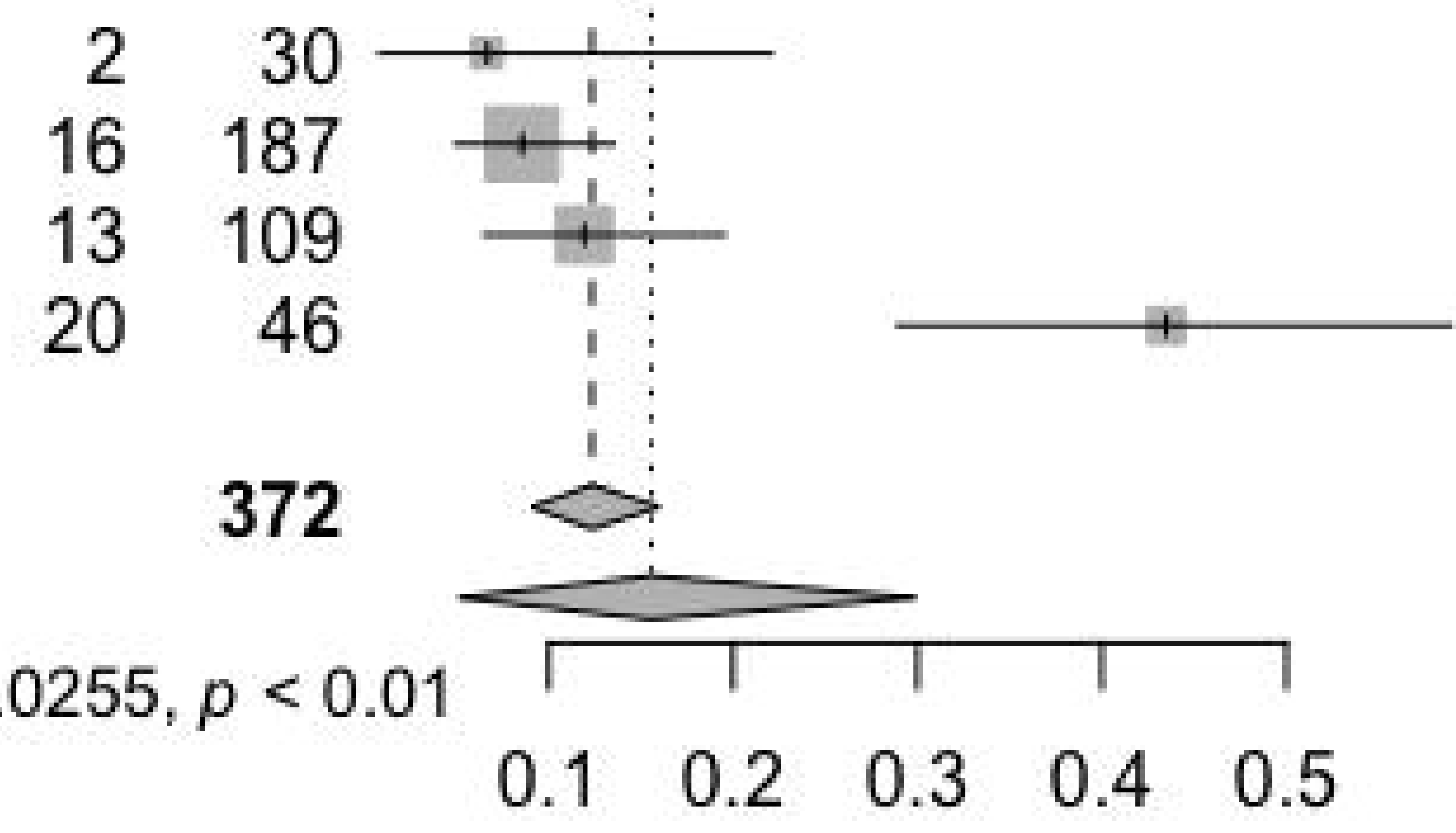
Events Total Rhinitis
Weight Weight

Proportion

$0.07[0.01 ; 0.22] \quad 8.2 \% \quad 21.9 \%$ $0.09[0.05 ; 0.14] \quad 50.1 \% \quad 27.5 \%$

$0.12[0.07 ; 0.20] \quad 29.3 \% \quad 26.6 \%$

$0.43[0.29 ; 0.59] \quad 12.4 \% \quad 23.9 \%$

$0.12[0.09 ; 0.16] 100.0 \%$

$0.16[0.05 ; 0.30]$

$-$

$100.0 \%$ 


\section{Study}

Sonnweber, et al.

Taboada, et al:

Garrigues, et al.

Fixed effect model

Random effects model

Heterogeneity: $I^{2}=0 \%, \tau^{2}=0, p=0.64$

\section{Events Total Sleep_disorders}

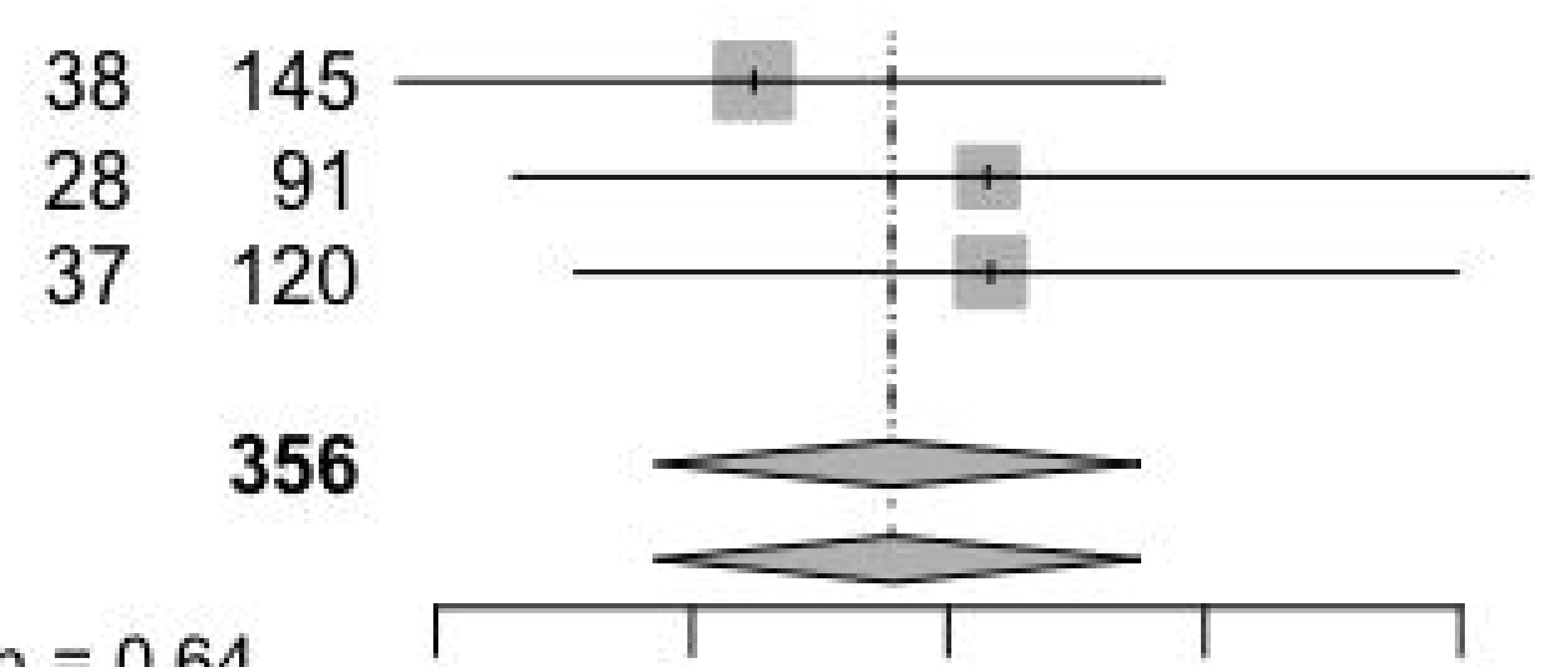

$\begin{array}{lllll}0.2 & 0.25 & 0.3 & 0.35 & 0.4\end{array}$
Weight Weight Proportion $\quad 95 \%-\mathrm{Cl}$ (fixed) (random)

$0.26[0.19 ; 0.34] \quad 40.7 \% \quad 40.7 \%$

$0.31[0.22 ; 0.41] \quad 25.6 \% \quad 25.6 \%$

$0.31[0.23 ; 0.40] \quad 33.7 \% \quad 33.7 \%$

$0.29[0.24 ; 0.34] 100.0 \%$

$0.29[0.24 ; 0.34] \quad-\quad 100.0 \%$ 
Study

Boscolo-Rizzo, et al.

Landi, et al.

Random effects model

Heterogeneity: $I^{2}=0 \%, \tau^{2}=0, p=0.85$

5
Events Total Sore_throat pain

$8 \quad 187$

(1)
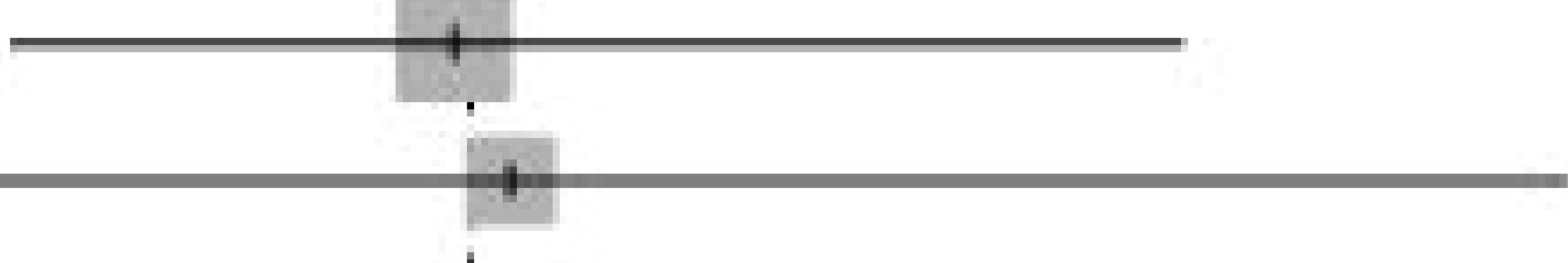

296 .

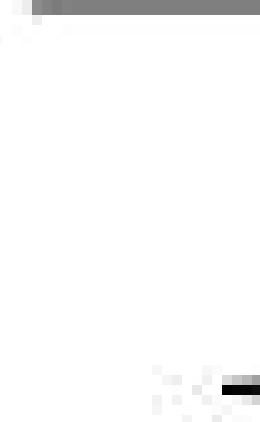
$\begin{array}{lllll}0.02 & 0.04 & 0.06 & 0.08 & 0.1\end{array}$
Proportion $\quad 95 \%$-Cl Weight

$0.04[0.02 ; 0.08] \quad 63.1 \%$

$0.05[0.02 ; 0.10] \quad 36.9 \%$

$0.04[0.02 ; 0.07] 100.0 \%$ 


\section{Study}

Zhao, et al. Jacobs, et al: Park, et al.

Fixed effect model Random effects model

Heterogeneity: $I^{2}=96 \%, \tau^{2}=0.0795, p<0.01$
Events Total Sputum

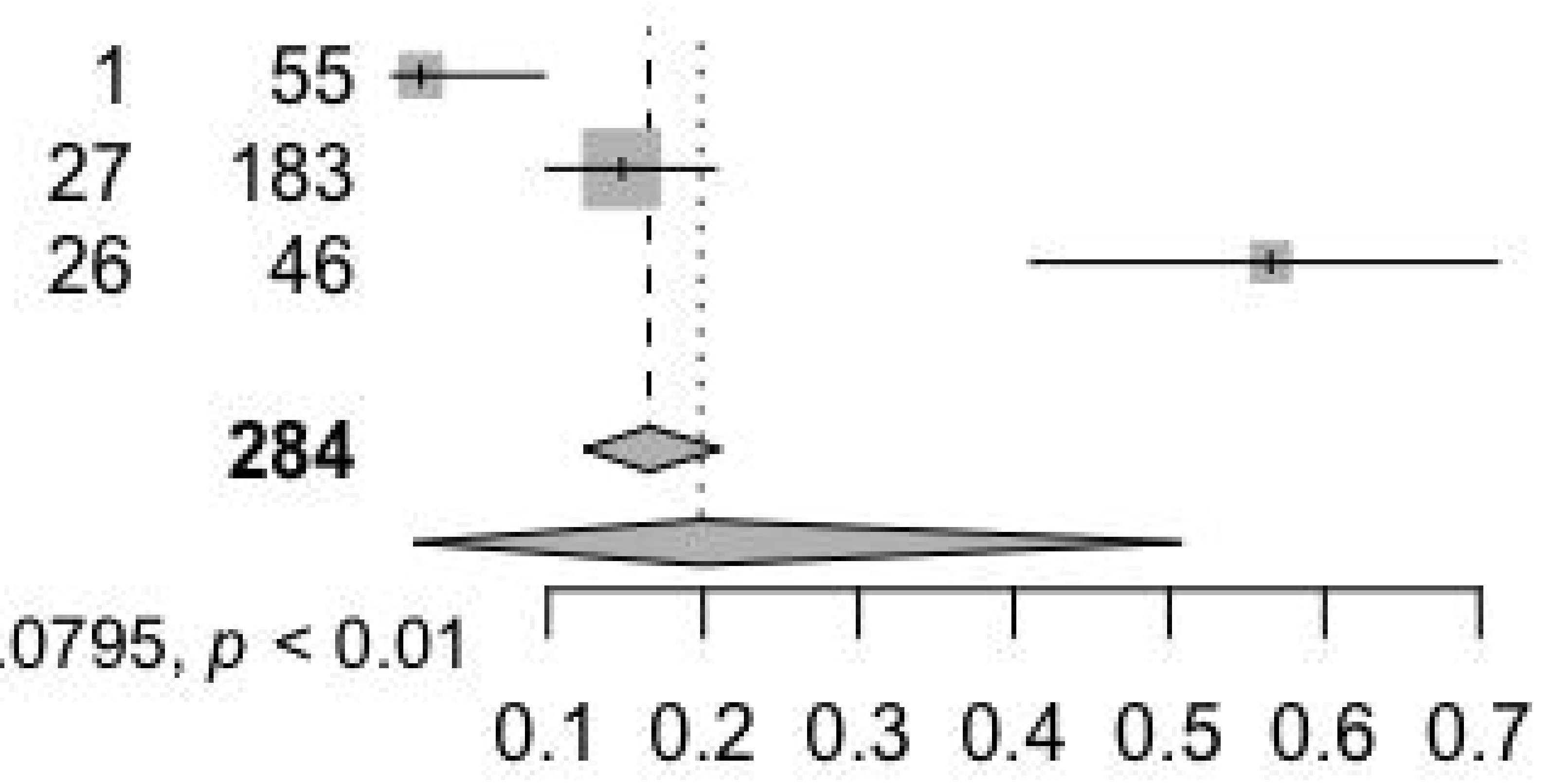

Weight Weight Proportion $\quad 95 \%-\mathrm{Cl}$ (fixed) (random)

$0.02[0.00 ; 0.10] \quad 19.4 \% \quad 33.0 \%$ $0.15[0.10 ; 0.21] \quad 64.3 \% \quad 34.3 \%$ $0.57[0.41 ; 0.71] \quad 16.3 \% \quad 32.7 \%$

$0.17[0.12 ; 0.21] 100.0 \%$ $0.20[0.01 ; 0.51] \quad--100.0 \%$ 